\title{
The Mathematical and Physical Theory of Rational Human Intelligence: Complete Empirical-Digital Properties; Full Electrochemical-Mechanical Model (Part I: Mathematical Foundations)
}

\author{
Leo Depuydt \\ Department of Egyptology and Ancient Western Asian Studies, \\ Brown University, Providence, USA \\ Email: leo_depuydt@brown.edu
}

Received April 18, 2013; revised May 3, 2013; accepted June 27, 2013

Copyright (C) 2013 Leo Depuydt. This is an open access article distributed under the Creative Commons Attribution License, which permits unrestricted use, distribution, and reproduction in any medium, provided the original work is properly cited.

\begin{abstract}
The design of this paper is to present the first installment of a complete and final theory of rational human intelligence. The theory is mathematical in the strictest possible sense. The mathematics involved is strictly digital — not quantitative in the manner that what is usually thought of as mathematics is quantitative. It is anticipated at this time that the exclusively digital nature of rational human intelligence exhibits four flavors of digitality, apparently no more, and that each flavor will require a lengthy study in its own right. The four flavors are as follows: 1) Selection Digitality (SelDi); 2) Nexus Digitality (NexDi); 3) Certification Digitality (CerDi); and 4) Supplement Digitality (SupDi). The provisional title of the forthoming second installment is "The Mathematical and Physical Theory of Rational Human Intelligence, Part II ("SelDi"): Selection Digitality and Probability as Its Perfect Analogue; Probability Redefined and Inverse Probability Inverted, or from Numbers of Balls to Numbers of Urns". The following account will be as explicit as possible in order to afford access to those who have only limited prior acquaintance with the overall topic. Succinctness has many virtues. But these virtues only go so far. The theory at hand describes the operations of a physical tool, the brain. In that regard, the theory is not only mathematical but also physical - more or less in the same way that, say, the theory describing the operations of an electric motor is both mathematical and physical. To the extent that it is designed to fully explain a physical phenomenon, the proposed theory of rational human intelligence can rightly be called a theory of physics and has been called so in the title of this paper. Then again, the physics involved is theoretical rather than applied. As a theory of physics, the theory of rational human intelligence contains a dominant mathematical component in the sense that J.-L. Lagrange's application of partial differential Equations to mechanical phenomena and J. C. Maxwell's application of such Equations to electromagnetic phenomena can be said to be purely mathematical. In fact, the Equations in question say nothing about what the actual phenomena that they describe really are. In that regard, J. C. Maxwell's theory of electromagnetism is not directly and bodily empirical. Nor is the proposed theory of rational human intelligence bodily empirical. Still, the intention at this time is that a future installment of the present description of rational human intelligence will include reflections on the empirical and bodily — which, in the case of the brain, means electrochemical — nature of rational human intelligence. What is more, as the description of a tool, the theory of rational human intelligence should be relevant to a practical side of physics, namely engineering. Since the tool in question is biological, the theory pertains more specifically to bioengineering. What follows in this first installment is not the theory of rational human intelligence itself. This installment is rather concerned with the mathematical foundations of the theory. Two critical preliminary concerns will need to be addressed. The first concern is with the kind of mathematics and the kind of mathematical notation that are needed and that will fully suffice to describe rational human intelligence in its entirety. The second concern is with the feasibility of a complete theory of rational human intelligence. First is the kind of mathematics and the kind of mathematical notation that are needed. It has already been noted above that the mathematics that is needed is entirely digital. The complete theory of rational human intelligence to be proposed below owes much if not most to the works of G. Boole and to the digital mathematics that is expounded in them. One reads on
\end{abstract}


occasion that G. Boole tried to establish how we think rationally and failed. Quite to the contrary, his efforts made it all the way there for the greater part. It will need to be clarified exactly what digital means. Evidently, G. Boole himself did not call his mathematics of the intellect digital. The term is derived from modern computer science, which owes it foundations to G. Boole. In any mathematical theory, the need is for a rigorously defined, consistent, and purely mathematical notation - in the way that the notation of, say, trigonometry is rigorously defined, consistent, and purely mathematical. In that regard, not only does what follows owe much if not most to G. Boole, but the notation that will be used in what follows is almost entirely his. Ever since J. Venn, no one has actively used G. Boole's notation of digital mathematics, even if this notation is discussed in all kinds of historicizing accounts. A principal aim of the present, first installment of a proposed complete theory of rational human intelligence is to discuss and illustrate G. Boole's notation and aver its singular solidity, veracity, and parsimony, argue in defense of its eminent superiority, and even expand it somewhat. The driving assumption will be that G. Boole's notation is by far the most effective. Meanwhile, a veritable Tower of Babel has been erected in the late nineteenth and early twentieth century in regard to the notation of symbolic logic, logics, propositional logic, and the like. There seem to be as many notations as prominent logicians. The working assumption is that none of these alternative notations is able to capture all of rational human intelligence in the way that G. Boole's notation can and actually does. Second is the feasibility of a complete theory of rational human intelligence. Hardly anything is known at this time about how rational human intelligence is propagated electrochemically in the brain. It will therefore be necessary to derive a theory about how rational human intelligence functions in the brain as a biological tool entirely from the outputs of the tool. Such a theory may be called a black box theory, in the sense that one has to derive what is in the box from what comes out of the box without being able to look inside the box. The box is black, in the sense of opaque, because one cannot look inside it. In fact, black box theories are common in physics. The theory of gravity is a black box theory. No one knows what gravity is. The black box theory that will be singled out in the final paragraph, $\S 25$, for detailed comparison, is J. C. Maxwell's theory of electromagnetism, because unlike the theory of gravity, it is more or less complete. And a more or less complete theory of rational human intelligence appears to be feasible. Some biographical notes on G. Boole and J. C. Maxwell will be presented in the same $\S 25$. The vicinity of the two men in place and in time makes it interesting to examine possible connections between them. Evidence of at least one direct connection between J. C. Maxwell and the work of G. Boole has been found, but so far no evidence of a personal encounter between the two men. There are otherwise many shared indirect connections between the two. Some of the most prominent ones will be noted. An interesting related matter is how machine intelligence compares to rational human intelligence. Both are fundamentally digital, but in different ways. It is hoped that the matter will be treated in a section of a future installment of the present effort or in a separate article entitled "Neurons versus Transistors: Macrodigitality vs. Microdigitality; Human Intelligence vs. Machine Intelligence". Among other recent efforts relating to the larger project of which the present paper is part, namely increasingly digitalizing the analysis of rational thought and language, are the following: 1) "Higher Variations of the Monty Hall Problem (3.0, 4.0) and Empirical Definition of the Phenomenon of Mathematics, in Boole's Footsteps, as Something the Brain Does", in Advances in Pure Mathematics (www.scirp.org/journal/apm), Vol. 2, No. 4 (July 2012), pp. 243-273; 2) "To Comma or Not to Comma: The Mathematics of the Relative Clause, All Types, via Boole and Venn", in International Journal of Intelligence Science (www.scirp.org/journal/jis), Vol. 2, No. 4 (2012), pp. 106-114. The CONTENTS of the 25 sections of the present article may be briefly summarized as follows. In Section $1(\S 1)$, the proposed theory of rational human intelligence is characterized as a theory of physics with a dominant mathematical component. It describes the mathematical laws by which the brain as a physical tool operates. In Section $2(\S 2)$, the proposed theory of rational human intelligence is characterized as a black box theory. Like the theory of electromagnetism, it infers what is in the brain in terms of rational human intelligence from what comes out of it without being able to look inside. In Section 3 ( $\$ 3$ ), the proposed theory of rational human intelligence is characterized as a complete theory. Section 4 (§4) describes what differentiates the physics of matter and motion from the physics of rational human intelligence. The physics of matter and motion involves quantity. The description of physical quantities necessitates the creation of units. The physics of rational human intelligence does not involve quantity. No units are therefore required. The minimal component is instead the attribute. In Section $5(\S 5)$, attributivity is defined. It is noted that both the physics of motion and the physics of rational human intelligence pertain to the mathematical description of certain distinct properties of mass. But whereas the physics of motion describes motion as a property of mass, the physics of rational human intelligence describes attributivity as a property of mass. Attributivity is the property of mass to exhibit features that make things or entities distinct from one another to the perceiving brain. In Section $6(\S 6)$, the attribute is defined. Attributes reside in the brain and are derived from the distinctive features that make up attributivity as a property of mass as a physical reality. Inside the brain, the attribute is a biochemical link between the imprint of an impression derived from such a distinctive feature of physical reality and an imprint of a sound pattern. In Section 7 (\$7), the attribute set or attribute class is defined. Set or class is a concept often used in mathematics. The present section clarifies how exactly the concept will be defined in the theory of rational human intelligence. The simple fact is that certain distinct features of things are shared by more than 
one thing. Common features naturally unite things into groups called sets or classes. In Section 8 (§8), the intensive interpretation of the attribute and the extensive interpretation of the attribute are compared with one another. Both interpretations are valid. The intensive interpretation has often been applied in philosophy, for example by G. W. Leibniz. But in the end, it is the extensive interpretation that is strictly mathematical. It is therefore the extensive interpretation that will be used exclusively in the theory of rational human intelligence. In Section 9 (§9), attribute mathematics is defined as one type of digital mathematics. In Section $10(\S 10)$, the four flavors of digitality that make up the totality of rational human intelligence are defined. In Section $11(\S 11)$, rational human intelligence is differentiated from rational human knowledge. In Section $12(\S 12)$, reflexivity is noted as a property of rational human intelligence. Rational human intelligence is a digital mathematical system that is able to describe itself. Sections $13(\S 13), 14(\S 14)$, and $15(\S 15)$ are concerned with the syllogism. For more than two millennia, since about Aristotle, no topic has been more closely associated with rational human intelligence than the syllogism. The syllogism has been widely viewed for the longest time, and still often is, as the epitome of rational and logical thought. However, it is proposed here that the syllogism has nothing to do with rational human intelligence. The best evidence is that one does not need to know anything about the syllogism to be endowed with rational human intelligence. Most people, in fact, do not. Then again, the syllogism is a valid concept. One problem is otherwise that the syllogism is incomplete and only reaches so far. The structure of the syllogism is fully digital and mathematical, like rational human intelligence itself. Because the syllogism exhibits close affinity with rational human intelligence, it will be most desirable to reach a proper understanding of how exactly the two relate to one another. In Section $13(\S 13)$, the syllogism is described as a pure digital mathematical structure and it is examined what exactly this means. In Section 14 (§14), one specific example of a supposed syllogism is analyzed in strictly digital and mathematical fashion. The syllogism in question is as follows. The first premise is that all philosophers are logical. The second premise is that an illogical man is always obstinate. The conclusion is, therefore, that some obstinate persons are not philosophers. It is established that, contrary to what has been assumed, this syllogism does not afford any conclusions whatsoever. In Section $15(\$ 15)$, the basic steps are presented towards a computer algorithm that makes it possible to solve any expansion of any syllogism to any degree. In Section $16(\S 16)$, it is noted that G. Boole conflates two designs in his work on digital mathematics. It has on occasion been wondered what he is doing, pure mathematics or describing how we think rationally. It appears that he is doing both without saying so. The confusion has led to a certain degree of misunderstanding of his true design. In Section 17 (§17), the quest for a deeper understanding of mathematics is described as being to a considerable degree a quest to heed the call of the adage "Know Thyself". In Section 18 (§18), G. Boole's digital mathematical procedure of "squaring" as a means of reducing two or more Equations to one is described. In Section $19(\S 19)$, an alternative to G. Boole's procedure of "squaring" is presented, namely multiplication by supplements. In Section 20 (\$20), examples of multiplication by supplements are presented. In Section 21 (§21), E. Schröder’s attempt to remove G. Boole’s $\frac{0}{0}$ from digital mathematics is rebutted. Sections $22(\S 22), 23(\S 23)$, and $24(\S 24)$ are designed to illustrate and clarify the significance and validity of $\frac{0}{0}$. In Section $22(\S 22), \frac{0}{0}$ is defined as fundamentally digital, as opposed to quantitative. A problem proposed by A. Macfarlane is adduced to illustrate $\frac{0}{0}$ and its quantitative counterpart as two facets of a single reality, as two sides of a single coin as it were. In Section 23 (\$23), G. Boole’s two symbols denoting indefiniteness, namely $\frac{0}{0}$ and $v$, are discussed and an attempt is made to resolve G. Boole’s confusing treatment of the two. In Section 24 (\$24), additional sophistication is lent to the interpretation of $\frac{0}{0}$ by considering the significance of the expression $\frac{0}{0} \times \frac{0}{0}$. Finally, in Section 25 (§25), J. C. Maxwell's theory of electromagnetism and the proposed theory of rational human intelligence are compared with one another in their capacities as black box theories and complete theories and possible associations between the person and work of J. C. Maxwell and the person and work of G. Boole are examined. This final section is in large part historical and can be read independently from all the other sections.

Keywords: Artificial Intelligence; Boolean Algebra; Boole's Algebra; Black Box Theories; Brain Science; Cognition; Cognitive Science; Digital Mathematics; Electricity and Magnetism; J.-L. Lagrange and Partial Differential Equations; J. C. Maxwell's Theory of Electromagnetism; Neuroscience; Non-Quantitative and Quantitative Mathematics; Physics; Rational Human Intelligence, Complete Theory of Rational Thought and Language 


\section{General Characterization of the Theory of Rational Human Intelligence as a Scientific Theory: A Theory of Physics with a Dominant Mathematical Component}

The design of this paper is to present the first installment of a complete and final theory of rational human intelligence. The present mode of inquiry has been applied in preliminary fashion in various ways in other publications [1-6]. It is not fully clear at this time how many installments will be needed to describe the theory in its entirety, but probably four, one devoted to each flavor of digitality (see §10).

There is nothing spiritual or philosophical, let alone mystical, about the theory that is to be proposed. It is a materialistic theory that describes a phenomenon of physical reality that is subject to absolute mathematical laws.

Being a scientific theory, with which domain of science does the theory of rational human intelligence exhibit the most affinity? The theory concerns the operation of the brain as a tool, more specifically the propagation of rational human intelligence as an event of nature. In that regard, the theory would appear to exhibit the closest affinity with physics.

Naturally, there are no sharp dividing lines between the domains of science. Thus, it is clear that physics has an extensive mathematical component. It is more or less the task of theoretical physics to define that mathematical component. It is more or less the task of applied physics to study the multiple concrete applications of the mathematical component of theoretical physics.

The theory at hand includes a description of the mathematics that explains rational human intelligence. In that regard, the theory belongs to the domain of theoretical physics. Owing to certain circumstances, its affinity with mathematics is especially strong and its affinity with applied physics somewhat weak at the present time.

\section{The Theory of Rational Human Intelligence as a Black Box Theory}

The inner workings of the brain are at this time only poorly understood. Does this mean that one should perhaps better abandon, or at least postpone, efforts to present a complete mathematical and physical theory of rational human intelligence? If a complete theory of rational human intelligence can be formulated at this time, it will need to be a so-called black box theory, that is, a theory that explains the inner workings of a system entirely based on the empirically observable outputs of the system.

J. C. Maxwell's theory of electromagnetism provides a splendid example of a black box theory of physics. The theory in question is more or less complete, and that while J. C. Maxwell did not even know what an electron is. J. C. Maxwell's theory is discussed in more detail in $\S 25$.

Only one of the four main forces of physical nature (strong, weak, electromagnetic, and gravity) is at this time more or less completely described and it is J. C. Maxwell's theory that has accomplished the feat. In that regard, I am firmly convinced that a complete theory of rational human intelligence is possible along the same lines. There is an abundance of information that can be derived from the outputs of the system.

If a complete mathematical description of rational human intelligence is possible, as I believe that it is, then what about the rational human intelligence as an actual physical event? It is evident that the event in question takes place inside the brain and is therefore electrochemical in nature. The accurate description of this event belongs to the fields of biochemistry and bioengineering.

What can be said about the actual physical event is at this time very limited. Then again, it will be proposed in the continuation of this effort that certain intimations about the physical nature of this event may be possible.

To the extent that the description of the system also leads to a desire to build an analogous system in a different platform, the theory of rational human intelligence acquires an affinity with the field of engineering. And to the extent that the brain is a biological tool and not a tool made of steel or any other lifeless material, the theory becomes of concern to the field that is now called bioengineering.

\section{The Theory of Rational Human Intelligence as a Complete Theory}

It will be useful to address right at the outset a potential major objection. How can a theory of rational human intelligence be more or less complete if so little is known about the brain?

It goes without saying that, if an essential requirement for completeness were a full account of the electrochemical operations of the brain, then no theory of rational human intelligence could at this time be anywhere near complete.

The best way to convey in which respect the theory of rational human intelligence that will be proposed is deemed to be more or less complete is again J. C. Maxwell's theory of electromagnetism. The theory is considered more or less complete even though, as was noted before, J. C. Maxwell was not aware of something now considered so elementary as the electron.

If knowledge of the electron were a requirement for $\mathrm{J}$. C. Maxwell's theory to be complete, then it would certainly not be anywhere near complete. Then again, it is 
generally accepted that J. C. Maxwell's celebrated Equations more or less completely account for all the essential workings of electromagnetism. These Equations provide a complete mathematical account of the physics of electromagnetism. In the same way, the design of the theory of rational human intelligence proposed below is to provide a complete mathematical account of rational human intelligence.

J. C. Maxwell's theory has been adduced by analogy not only to point to the feasibility of success on the part of a black box theory but also to the possibility that a black box theory is complete.

\section{Quantity and Units in the Physics of Matter and Motion}

The theory of rational human intelligence has been characterized above as a theory of physics. The brain is a tool and the propagation of rational human intelligence is a physical event. At this time, the human brain is the only type of tool in which rational human intelligence is propagated. The design of what follows is to describe rational human intelligence as a physical event or physical events. The need is therefore for describing the mathematical relations that define the propagation of rational human intelligence as physical events. In that regard, the theory of rational human intelligence has to be profoundly mathematical, just as all theories of physics are.

In order to better envision the theory of rational human intelligence as a theory of physics, it will be useful to examine how the well-known theories of physics are constituted and compare these theories of physics with the theory of rational human intelligence.

J. C. Maxwell played a crucial role in the standardization of the units of physics in the nineteenth century and he wrote a celebrated essay on the nature of physics entitled "Matter and Motion" [7]. It will be useful to seek inspiration from these pioneering efforts pertaining to the standardization and definition of physics. J. C. Maxwell would be the first to agree, I believe, that "science is always most completely assimilated when it is in the nascent state" [8].

What does physics study and how does what it studies compare to what the theory of rational human intelligence studies as a theory of physics? It will be useful to distinguish between that which physics pertains to and which facet of what it pertains to it studies. Physics pertains to physical bodies, that is, matter. Physics studies certain properties of those physical bodies. These properties can be summed up by means of a single word: motion. It does not come as a surprise that J. C. Maxwell called his pioneering essay on the nature of physics "Matter and Motion". Matter may be called the object of physics. But what physics actually studies is the behavior of this object, more specifically motion. Consequently, motion may be called the study object of physics.

The motion of physical bodies is all about how physical bodies relate to the two dimensions of the human condition: space and time. Features of motion include Displacement, Velocity, Momentum, Acceleration, Force, Work, and Power.

These features of motion all have a fundamental characteristic in common. They all exhibit quantity. There is no escaping the impression that they are all capable of increase or diminution. They can get bigger or smaller. It may already be anticipated right now that the mathematics and physics of rational human knowledge will not involve quantity. In that regard, it sets itself apart from most other fields of mathematics.

How can one describe the quantity of any phenomenon of physical reality very much in general? It is obvious that one cannot describe all of quantity all at the same time. The need is for describing partial quantities.

In that regard, the problem is that quantity does not have discrete parts. Quantity does not have any subcomponents of a certain size that are given naturally in nature. In other words, the increase or diminution of quantity is continuous. As a quantity increases or decreases, it at no point reaches a point that is given as a natural subdivision in nature. I am disregarding the quanta of quantum theory on the subatomic level. They are not needed to formulate the classical physics of matter and motion.

The evident solution to describe quantity has always been and will always need to be to completely randomly pick a certain quantity and measure all the other quantities by establishing either how often that certain quantity fits into the other quantities or what part of that certain quantity fits into the other quantities. That certain quantity is counted as 1 . It is therefore called the unit, from Latin unus "one". If that quantity fits twice into another quantity, then that other quantity is counted as 2 and contains 2 units.

It may already be anticipated right now that the physics of rational human knowledge does not involve units because it does not involve quantity. But before turning to what exactly in the description of the physics of rational human intelligence corresponds to quantity in the description of the physics of matter and motion, it will be useful to add some detail about the description of quantity in the physics of matter and motion to afford a better contrastive comparison with the physics of rational human intelligence and add clarity to the latter.

The physics of matter and motion involves three types of quantities. One of the three quantities pertains to mass. It needs to be possible to establish how much of it there is. The two other quantities pertain to the motion of mass. The two dimensions of motion are the same as the two 
fundamental dimensions of the human condition, space and time. Motion is a change in space that happens over time. It is therefore necessary to create three units, one for mass, one for length (in one, two, or three dimensions), and one for time. Three examples of units of mass, space, and time are the kilo, the meter, and the second.

All the quantities of the physics of matter and motion are compounds of these three units. Displacement is expressed in just meters, $m$; Velocity, in meters over seconds, $\mathrm{m} / \mathrm{s}$; Momentum, in kilos multiplied by meters over seconds, $k \times m / s$; Acceleration, in meters over seconds squared, $\mathrm{m} / \mathrm{s}^{2}$; Force, in kilos multiplied by meters over seconds squared, $k \times m / s^{2}$ (that is, in newtons); Work, in kilos multiplied by meters squared over seconds squared, $k \times \mathrm{m}^{2} / \mathrm{s}^{2}$ (that is, in joules); and Power, in kilos multiplied by meters squared over seconds cubed, $k \times \mathrm{m}^{2} / \mathrm{s}^{3}$ (that is, in watts).

If object and study object of the physics of matter and motion are matter and motion, then what are the object and the study object of the mathematics and physics of rational human intelligence?

It has already been anticipated above that quantity does not play a role in rational human intelligence. There is therefore no need for countable units in order to describe rational human intelligence.

But before turning to how rational human intelligence is described, if not in terms of units, it will be useful to address a potential objection to the notion that rational human intelligence does not have anything to do with quantity.

It is a fact that knowledge exhibits quantity in two respects. First, it can grow and it can decrease. Second, part of knowledge concerns the knowledge of quantity, including numbers. Does this mean that rational human intelligence somehow involves quantity?

It does not. Knowledge needs to be distinguished from intelligence. Intelligence is a vehicle or tool that can be used in the service of an increase in knowledge. And to this knowledge belongs the knowledge of numbers. As a tool, intelligence itself does not exhibit quantity.

It is quite likely that human intelligence has become more complex and will continue to become more complex in the course of human evolution. No one would assume that the Neanderthals were as intelligent as we are. Does this then mean that intelligence somehow exhibits quantity? It does not. The principles that govern rational human intelligence have presumably remained independent of quantity throughout.

Any likely increase in the complexity of intelligence over time does mean that intelligence would have grown more powerful as a tool. Accordingly, the acquisition of knowledge would have become more efficient and more sophisticated in the course of human evolution.

\section{Absence of Quantity and Presence of Attributivity in the Physics of Rational Human Intelligence}

Matter has been called above the object of the physics of matter and motion. And motion has been called its study object. What, then, are the object and the study object of the physics of rational human intelligence?

It appears that the object of rational human intelligence is the same, that is, mass or physical things.

It goes without saying that many things are not physical. Examples are friendship and wisdom. However, it seems obvious that the brain conceives of non-physical things as being just as physical as physical things. In that regard, non-physical things are conceived as exhibiting mass and being subject to space and time just as much as physical things are. Accordingly, it is possible to speak of great wisdom in terms of space and of a long friendship in terms of time. In terms of mass, friendship can be called a burden. Like physical things, wisdom and friendship can be imparted, received, expanded, and so on.

The same mathematical laws of rational human intelligence will therefore apply to things such as wood and iron just as much as they do to things such as friendship and wisdom.

Whereas the physics of matter and motion and the physics of rational human intelligence share the same object, they do not share the same study object. Each studies a different property of matter. Physics studies how matter behaves in relation to space and time. The focus of the study of rational human intelligence is on a different property of matter. This property may be called attributivity.

Attributivity is the property of exhibiting certain distinctive features that can be perceived by the brain as what will be called here attributes. There is a fundamental difference, however, between motion and attributivity as study objects.

\section{The Attribute as the Fundamental Component of the Physics of Rational Human Intelligence}

The mathematics and physics of rational human intelligence is all about attributivity as a mathematical notion. As contrasted with motion, attributivity does not exhibit quantity.

As a quantity, motion consists of units. Units are not given in physical reality. They are a construct of the brain.

By contrast, attributivity consists of attributes. Attributes are likewise a construct of the brain. But they do originate in features of physical reality. As part of rational human intelligence, they are acquired properties of 
the brain that are reflections of features of physical reality outside the brain.

More attributes do not make a thing larger in size, as more units would. Rather, more attributes make a thing more distinctive. Again, attributivity does not exhibit quantity.

What, then, are attributes? As has been said, attributes are acquired properties of the brain that originate in a certain facet of reality outside the brain. What is this facet of physical reality outside the brain?

It is evident that physical reality is not uniform. It consists of a countless variety of things, which will also be called entities. All matter may be subject to the same laws of motion. But all matter is not uniform in appearance. If one item of matter differs from another item, then there must be one or more distinctive features exhibited by the former that is not found in the latter and typically vice versa. Such distinctive features are the origin of the attributes. It is such distinctive features that lie at the origin of the existence of what may be called attributes in the brain.

The attributes presumably originate in certain properties of physical reality outside the brain. But as a component of rational human intelligence, the attributes are acquired properties of the brain. The brain of a newborn child is entirely free of such attributes. The attributes are part of the rational operation of the brain as a biological tool.

Attributes consists of two sides or two parts. It is the connection between the two sides that makes the attribute.

How are attributes acquired by the brain? The brain is able to take note of the distinctive features of different items of matter. These distinctive features of reality make an impression on the brain. As a result, each distinctive feature leaves a biochemical imprint of some kind in the brain.

These imprints can be visual or auditory or relate to any other of the senses by which the brain communicates with that which is outside itself. This much for one side or one part of what makes an attribute. What about the second side or part?

The brain is able to associate a distinctive imprint of a feature of reality with a distinctive sound pattern. That distinctive sound pattern is also stored as an imprint in the brain. This imprint can steer the speech organs to pronounce the sound pattern whenever such a pronunciation is needed.

But it is the link between the imprint of a distinctive feature of a certain item of matter and the imprint of a sound pattern that makes an attribute. That link is itself an imprint.

Again, all three types of imprints are assumed to be of the biochemical kind.
Whenever the imprint of a distinctive feature of a certain item of matter is linked with an imprint of a sound pattern, an attribute is born. The attribute is the fundamental component of rational human intelligence. The design of this paper and forthcoming papers is to describe the mathematics of the attributes that make up rational human intelligence. The mathematics of the attributes is not quantitative. This type of non-quantitative mathematics can be called attribute mathematics or attributivity mathematics. Attribute mathematics is fundamentally digital. The concept "digital" is defined in more detail further below.

Consider as an example of a distinctive feature the visual impression of a dog (hairy, walks on four legs, generally cheerful, and so on).

No two people have the exact same visual impression of what a dog is. But the visual impression of a dog is sufficiently distinct or different from any other possible impression, whether visual or auditory or related to any other of the senses, that two or more people can readily agree that they have the same impression in mind when communicating. Difference is everything.

In the brains of English speakers, the imprint of the sound pattern $d o g$ is linked to the imprint of the visual impression of a dog as the reflection of a distinctive feature of matter.

The brains of French speakers link the imprint of the sound pattern chien to the same visual imprint.

The fact that the visual imprint is the same but the sound pattern imprint differs in a sense demonstrates the independent existence of the two imprints.

The brains of German speakers link the imprint of the sound pattern Hund to the imprint of that very same visual impression.

In sum, there is something profoundly dual about language and thought. If one includes the link between sound pattern and notion, then there is something profoundly ternary about language and thought.

The independence and separate physical existence of the imprint of a certain sound pattern and the imprint of the visual impression of a dog is guaranteed by the very fact that different sound patterns can be linked to the same visual impression in different languages. The sound pattern can only change while the visual impression remains the same if the sound pattern and the visual impression are distinct from one another and lead independent existences.

In other words, the sound pattern could not change and the visual impression at the same time stay the same if the two were the exact same thing. If such a thing were possible, then the very same single thing would at the same time both change and not change or remain the same. It is impossible for something to simultaneously become something else and remain the same. 
It must be assumed that the following three items have their distinct biochemical imprint in the brain: 1) the sound patterns $d o g$, chien, and Hund; 2) the visual notion of a dog; and 3) the link between 1) and 2). It is safe to assume that these imprints will some day be found and empirically verified. They are fundamental to the operation of the brain as a physical tool.

But until these imprints are found in the flesh, as it were, the theory of rational human intelligence will remain a black box theory in the sense that J. C. Maxwell's theory of electromagnetism is a black box theory.

Additional links that will need to be found inside the physical brain and described are the following: 1) those between the imprint of the sound pattern in the brain and the speech organs - mouth, tongue, and so on - that can activate the sound pattern; 2) those between the imprint of the sound pattern and the writing hand that can give written expression to the sound pattern; and 3) those between the eyes and the brain that first store the visual impression of a dog in the brain and also make it possible to recognize a dog for what it is once the visual impression in question has been stored in the brain.

In sum, the study of rational human intelligence is in the first place the study of the brain. But it is secondarily also the study of how the brain communicates with what is outside itself.

It is not necessary for the brain to activate or exteriorize the imprint of a sound pattern by means of the speech organs for the imprint to be stored inside it. The biochemical imprint of a sound pattern such as $d o g$ is ready to be triggered any time, as it were, by an act of pronunciation whenever the visual impression of a dog arises in the mind. Until such an occasion arises, the imprint is inactive or dormant, yet very much present.

This approach involving 1) impression, visual or other, 2) sound pattern, and 3) link between the two will not in the least be strange to anyone even remotely familiar with F. de Saussure's foundational theory of linguistics of the early twentieth century. As is well-known, F. de Saussure calls an impression a "signified"; a sound pattern, a "signifier"; and the link between the two, a "sign" (in French, signe linguistique "linguistic sign"). In F. de Saussure's linguistic terminology, the "sign" is not a physical thing, say like a traffic sign, but rather a link inside in the brain between the signified and the signifier.

G. Boole was in fact fully in possession of this conception of signified, signifier, and sign a few decades before F. de Saussure [9]. But none of the many, many who have commented on F. de Saussure's celebrated theory and its antecedents make reference to G. Boole, as far as I know.

As F. de Saussure and G. Boole both well understood, there is a profoundly social aspect to attributes or signs. All the speakers of English tacitly agree to call a dog a dog, that is, to link the visual impression of a dog to the imprint of the same sound pattern, namely $d o g$. They do not really have to. But they do so unawares. However, if one would not, one cannot be understood by other English speakers.

As F. de Saussure and G. Boole also well understood, there is something at the same time profoundly arbitrary and profoundly mandatory about attributes or signs. It does not really matter what one calls a dog. The sound pattern $d o g$ has no necessary relation with the animal in question. The relation is completely arbitrary. Then again, if one wishes to be understood by speakers of English, then the relation becomes mandatory.

Motion is a property of mass. The relation between mass and motion leaves an imprint on the brain. It is then upon the brain to establish the mathematical laws that characterize the physics of mass and motion. These laws involve quantity.

Like the relation between mass and motion, the relation between mass and attributivity leaves an imprint on the brain. It is then upon the brain to establish the mathematical laws of the physics of mass and attributivity. Part of those laws is also the mathematics of rational human intelligence. As opposed to the mathematics of mass and motion, the mathematics of mass and attributivity do not involve quantity.

Substantives or nouns and adjectives both signify attributes of things. The difference between the two is that substantives or nouns typically refer to what may be called clusters of attribute components viewed as a single, but composite, attribute whereas adjectives signify single attributes.

For example, the noun "tree" signifies all the multiple attributes that collectively make up a tree as distinct from any other kind of thing. There are obviously several attributes that together make a tree distinct from other kinds of things. The noun "tree" refers to all these attribute components as a single whole, that is, it refers to a single compound attribute.

By contrast, the adjective "green" typically signifies a single attribute that makes a certain kind of thing distinct from all other kinds of things.

\section{Attributes and Attribute Sets or Classes}

Sets or classes have played a prominent role in mathematics for well over a century now. As far as their role in quantitative mathematics is concerned, many have assumed that sets have led mathematics into paradise. As a result, it seems difficult to imagine quantitative mathematics without sets. Sets seem ubiquitous. Still, my own sense is that quantitative mathematics might well do perfectly fine without sets. But this is not the place to elaborate on this perception. 
In any event, the present section is not concerned with the role of sets or classes in quantitative mathematics but rather with their role in attribute mathematics. The need is for providing an exact mathematical definition of the class or set. There is evidently a widespread sense that classes play a role in logic. But what exactly are classes in attribute mathematics?

Consider attributes such as the noun "tree" or the adjective "yellow". These attributes serve to identify a thing as distinct from all other possible things. But at the same time, something else is immediately apparent in an axiomatic way, that is, in a way that is so self-evident that one cannot possibly doubt it, in the way that the axioms of mathematics are accepted without proof.

That something else is the fundamental fact of physical reality that a certain distinctive feature may be shared by more than one thing. For example, there is more than one thing that is a tree and there is more than one thing that is green.

It follows that two or more things exhibit an affinity or are members of a group of things that all exhibit a certain distinctive feature in reality and therefore a certain attribute in the brain. Nothing seems more natural. Nothing is easier to accept as an axiom of attribute mathematics. The need arises for a term to denote a group of things all sharing a single distinctive feature and therefore a single attribute in the brain. The time-honored and more or less synonymous terms "class" and "set" both present themselves as ideally suited to fill this need. It follows that, in the propagation of rational human intelligence, an attribute can refer to an entire group of things, called a class or set. But rational human intelligence also includes the means of referring to certain members of the class or set. Details regarding this matter will follow in later stages of this effort to describe rational human intelligence in its entirety.

In conclusion, the attributes are the crucial buildingblocks of rational human intelligence. The rest of rational human intelligence consists of operations, actions that the brain performs with the attributes as building-blocks.

It is possible to think of the attributes independently of any things or events to which they are attached. An example is the attribute "yellow". It is possible to contemplate the attribute "yellow" independently from anything that is yellow and establish what is known about it. However, such an effort is all about knowledge and not about intelligence. It is not possible to propagate rational human intelligence without treating attributes as properties of things or entities.

\section{The "Extensive" Function of Attributes}

In past centuries, there have been two fundamentally different ways of handling attributes. They may be called, with J. Venn, the extensive interpretation and the intensive interpretation of attributes.

J. Venn devotes a detailed discussion to the intensive interpretation as compared to the extensive interpretation [10]. But the main point of his discussion is the observation that it is the extensive interpretation of attributes that prevails in G. Boole's algebra-and hence also in the mathematical and physical theory of rational human intelligence. It will be useful to show definitively that only the extensive interpretation is strictly mathematical.

Then again, that does not mean that the intensive interpretation is devoid of meaning or usefulness. It has its rightful place in the study of the human condition. But it is at home in the domain of knowledge rather than in the domain of intelligence.

"Extensive" and "intensive" are somewhat abstract as terms. Their abstract character is in danger of obscuring how fundamentally different the two are. The difference between them may be defined as follows.

In the extensive interpretation, attributes are always interpreted as properties of physical things or entities. Their meaning extends to, and includes, the actual things that exhibit them. As was noted above, abstract things such as "wisdom" and "friendship" are for all practical purposes treated by the brain as physical things and therefore function as such in rational human intelligence.

In the intensive interpretation, attributes are considered in and by themselves, independently from the actual physical entities with which they associated or to which they are attached as it were.

An example may serve to clarify the distinction between the two. Consider the two attributes "rational" and "animal". According to the well-known classical definition, man is a rational animal.

In the philosophical interpretation of this definition, the attributes "man", "rational", and "animal" are intensive. In other words, they are considered by themselves independently from any entities to which they are attached.

According to the above definition, if one adds the attribute "rational" to "animal", one obtains "man". The attribute "rational" does not stand for the class or set of all the things that are rational, as it would in G. Boole's algebra. By the same definition, if one subtracts the pure attribute "rational" from "man", one obtains "animal".

A prominent practitioner of the intensive interpretation was G. W. Leibniz. I owe the following reference to J. Venn, whose knowledge of the history of logic before and during his time was unparalleled [11]. G. W. Leibniz formulates the above definition as follows [12]:

$$
\text { Homo - Rationalis } \infty \text { Brutum }
$$

That is, "Man minus (-) rational equals $(\infty)$ brute".

G. W. Leibniz uses the symbol " $\infty$ " as an equivalent of 
"=" and by "brute" he presumably means more or less the same as "animal".

The way in which G. W. Leibniz formulates the definition gives it the aura of mathematics. But mathematical it is not, in the way that G. Boole's algebra and the mathematics of rational human intelligence are mathematical. Then again, that does not mean that G. W. Leibniz's intensive interpretation of attributes is devoid of meaning.

In the interest of fully clarifying the relation between the intensive interpretation of attributes and the extensive interpretation of attributes, certain questions are in need of an answer:

1) What would be the strictly mathematical interpretation of the definition "Man is a rational animal" according to G. Boole's algebra and the mathematics of rational human intelligence?

2) What in G. Boole's algebra and in the mathematics of rational human intelligence would be the strictly mathematical interpretation of subtracting "rational" from "man" as G. W. Leibniz does?

3) What is the purport of G. W. Leibniz's interpretation if it is not part of the mathematics of rational human intelligence?

The following answers to these questions may be proposed:

1) The obvious way of representing a statement such as "Man $(m)$ is a rational $(r)$ animal $(a)$ " in G. Boole's algebra is as follows:

$$
m=r \times a .
$$

G. Boole generally omits the symbol " $x$ " and hence would normally write as follows:

$$
m=r a \text {. }
$$

This Equation implies that "man" encompasses all that is both rational and an animal and not just an indefinite amount of what is both rational and an animal. If the latter were the case, then the Equation would rather be as follows:

$$
m=m \times r \times a \text {. Or also: } m=m r a .
$$

In the above Equations, " $x$ " signifies the Boolean operator AND.

But what does the definition "Man is a rational animal" mean if it is stated in G. Boole's algebra as it has been above?

It will be good to compare the definition with a similar statement exhibiting the same structure, such as "The Flemish $(f)$ are the Dutch-speaking $(d)$ Belgians (b)". There are Dutch speakers that are not Belgians and Belgians that are not Dutch speakers. The Flemish are all those that are both, nothing more, nothing less. The statement would be represented as follows in G. Boole's algebra:

$$
f=d \times b \text {. Or also: } f=d b .
$$

The only interpretation of this statement that makes sense is evidently the extensive one. There are entities that are Dutch-speaking and there are entities that are Belgian. The Flemish constitutes the overlap between the two, that is, entities that exhibit both attributes.

The mere fact that this is the interpretation that everyone expects to be valid if one executes a simple Boolean search with any search engine on the Internet already sufficiently indicates that it is the extensive interpretation that is valid in non-quantitative mathematics or attribute mathematics, the type of mathematics that describes rational human intelligence.

If one applies this exact same extensive interpretation to "Man is a rational animal", then there ought to be entities that are rational and entities that are animals and man constitutes the overlap between the two, that is, entities that exhibit both attributes.

This extensive interpretation can only make sense if "animal" is understood to include "man". This is in fact one of the possible meanings of "animal". However, it is not how the word "animal" would normally be understood in everyday speech.

Still, I suspect that, outside of the realm of philosophy, the word is as a rule understood in this way in the definition "Man is a rational animal", or also "Human beings are the rational animals". The reason is that it is only natural to interpret "Man is a rational animal" in the same way as "The Flemish are the Dutch-speaking Belgians". This interpretation follows the mathematical laws of rational human intelligence. The interpretation therefore imposes itself to anyone not familiar with the history of philosophy.

Then again, that is not how philosophers have typically understood the statement "Man is a rational animal". In the case of G. W. Leibniz, this is obvious from two facts. First, he uses brutus "stupid" instead of "animal" and therefore clearly refers to animals at the exclusion of human beings. Second, he subtracts "rational" from "man" and therefore by his own reasoning obtains something that is devoid of reason and that something cannot therefore include human beings.

2) What is the result of subtracting "rational" from "man" in G. Boole's algebra?

In the mathematics of rational human intelligence, "rational" and "man" denote two sets or classes of entities exhibiting the two attributes in question. After all, in physical reality, attributes do not exist independently from the things or entities to which they are attached. It follows naturally that, in subtracting or removing rational entities from the set of human beings, one cannot remove anything that is not a human being from the human beings. What is left after removing the rational human be- 
ings from all the human beings are all the irrational human beings, whatever that may be.

However, if one accepts on the basis of the definition "Man is a rational animal" that man is always rational, then there are no irrational human beings. Accordingly, after subtracting rational human beings from the human beings, nothing is left. Likewise, if one subtracts all the Dutch-speaking Flemish from the Flemish, one is left with nothing. In that regard, it is possible to subtract "rational" from "man" in the extensive interpretation. But it makes little sense if human beings are all rational.

G. W. Leibniz subtracts "rational" from "man" and obtains "animal", as follows:

$$
m-r=a \text {. }
$$

It follows by his own reasoning that

$$
m=r+a \text {. }
$$

That is, "man" is obtained by adding "rational" to "animal".

In G. Boole's algebra, the symbol "+" stands for the OR function. The mathematical meaning of the last Equation is therefore "Man is either rational or an animal or both at the same time". This meaning is what one expects from the OR function in any Internet search. It appears again that G. W. Leibniz's intensive interpretation is not that of the non-quantitative mathematics that explains rational human intelligence.

Judging from mere Boolean searches on Internet search engines, it would appear evident that the extensive interpretation of attributes is the mathematical way.

The deeper reason of the fact that the extensive interpretation is valid in mathematical terms and not the intensive interpretation would appear to be that, as has already been noted above, attributes do not exist independently of things or events in physical reality. The mathematics of attributes, like any type of mathematics, ought to be a tool that describes physical reality as it is. In the intensive interpretation of attributes, attributes are viewed independently from the things or events to which they are attached. Attributes existing independently from things or events are not part of physical reality.

When G. W. Leibniz follows the philosophical tradition by subtracting "rational" from "man", he is in effect detaching an attribute from its thing or entity. The result is an attribute without an entity, something that does not occur in physical reality.

3) The philosophical approach exemplified above by means of the views of G. W. Leibniz is otherwise not devoid of purport. But any results deriving from this approach are better classified as knowledge rather than as intelligence. Intelligence is the tool that serves the function of accruing knowledge. Describing the mathematical and physical laws to which this tool is subject is the design of the present paper and follow-up papers. The phi- losophical approach is an application of intelligence as a tool, not the tool itself.

In the philosophical approach in question, intelligence is applied to the following evident fact: different things and different events exhibit different attributes. Differences invite comparison. One interesting result of such comparison is the observation that things can have certain attributes in common and other attributes not. Especially intriguing are instances in which two things or two events have most every attribute in common except for just a few or even just one. For example, human beings and animals have most everything in common, but not completely everything. The focus naturally turns to that which the two do not have in common. In that regard, it appears that animals cannot think. It is tempting to ponder the notion that, if one could just add rationality to animals, they would be like human beings, at least if one discards certain differences in appearance between the two.

Being aware of the difference between human beings and animals is part of knowledge, in the same way that knowing the differences between the various flora and fauna of a certain nation is part of knowledge. However, to ask what it would take in terms of addition or subtraction of attributes to make one species exactly into another species, or more like another species, is a pure product of the imagination. There is no way that one can subtract rationality from human beings to make them more like animals. There is no way in which one can add wings to a pig and make it into a bird. Still, if a pig had wings and could fly, it would technically be a bird.

The conceptual feats involved in the intensive interpretation of attributes can be accomplished only as pure exercises of the intellectual imagination. Such exercises consolidate one's knowledge of the difference between the two. It is therefore not totally without a certain usefulness. But it bears no relation to physical reality. It cannot, therefore, be the object of a mathematical and physical analysis.

\section{9. "Digital Mathematics" as a Name for Attribute Mathematics}

The mathematics of rational human intelligence is nonquantitative. Nothing gets bigger or smaller. In fact, in $\mathrm{G}$. Boole's algebra, $x \times x=x\left(\operatorname{not} x^{2}\right)$ and $x+x=x(\operatorname{not} 2 x)$. There is no increase in size.

The mathematics in question has been called attribute mathematics above. But it can also be called digital mathematics.

"Digital" is a term that is now ubiquitous. The term is short for "binary digital". "Binary digital" refers to a type of numeration using the base 2 and therefore only the digits 0 and 1 . In binary numeration, the numbers $1,2,3$, 
4, and 5 of decimal operation correspond to $1,10,11$, 100 , and 101.

By extension, the term "digital" has come to be used to denote any system in which there are only two values. For example, the transistors of a computer are either On or Off. An electrical current does or does not run through them. The operations of a computer may therefore be called digital.

The mathematics of rational human intelligence is likewise digital. What are the two values in question? Attribute mathematics is all about items of mass or things or entities exhibiting attributes. It follows that there are exactly two options. An entity may or may not exhibit a certain attribute. In other words, things can exhibit two possible states pertaining to a certain attribute. An attribute can be either present or absent. An example of an attribute is "green". An item of mass can be either green or not green. Again, in the mathematics of rational human intelligence, attributes are properties of things or entities.

It may be tempting to assume that the present state is more interesting than the absent state. This may well be the case when it comes to knowledge. There are so many countless attributes that an item of mass may not exhibit. It is therefore much more interesting to know which attributes it does exhibit.

However, as far as the mathematics and physics of rational human intelligence is concerned, the present state and the absent state are equally important in the sense that both are equally indispensable to the propagation of rational human intelligence.

How present states and absent states interact mathematically and physically in the propagation of rational human intelligence will be described in detail later. It will become clear that intelligence has everything to do with a loss or deficit or diminution of entropy, to borrow a term from thermodynamics, on the part of attributes. A deficit of entropy corresponds to a gain in organized behavior. It is this deficit that for the most part makes rational human intelligence possible.

All attribute mathematics is digital mathematics but not all digital mathematics is attribute mathematics. The mathematics of an electronic circuit board in a computer is also digital mathematics. But it is not attribute mathematics.

\section{The Four Flavors of the Digitality of Rational Human Intelligence}

As far as I can see at the present time, there are four kinds or manifestations or flavors in the digitality of rational human intelligence. They may be called as follows:

1) Selection Digitality (SelDi, Sel.Di, or sel.di);

2) Nexus Digitality (NexDi, Nex.Di, or nex.di);
3) Certification Digitality (CerDi, Cer.Di, or cer.di).

4) Supplement Digitality (SupDi, Sup.Di, or sup.di).

Each of these flavors of digitality will need to be discussed in great detail in future papers. It is anticipated at this time that at least one long paper will need to be devoted to each. Each flavor is quite distinct from the other and the aim of what is to come is to elucidate their nature.

It is only Selection Digitality that the brain presently shares with the computer. Selection Digitality revolves around the Boolean operators AND, OR, and NOT, perhaps best known in everyday life from searches on the Internet. All digital functions of the brain as a tool are strictly subject to the more general laws of digital mathematics applicable to rational human intelligence, just as the electrical functions of an electric motor are strictly subject to the more general laws of physics applicable to electricity and magnetism.

What follows owes an enormous debt to G. Boole. It is in large extent a specific kind of extended implementation of his thoughts. Much is also owed to the various ways in which J. Venn was able to make G. Boole's thoughts more accessible.

Then again, there are additions and specifications in what is to come. As far as I can see, 3) Supplement Digitality (SupDi) and 4) Certification Digitality (CerDi) cannot be derived from G. Boole's or J. Venn's writings. Some manifestations of Nexus Digitality as part of Selection Digitality also go beyond what G. Boole and J. Venn have had to say.

The task of logical reasoning is performed by the digital functions 1), 2), and 3), and more narrowly by 2) and 3). G. Boole and J. Venn discuss 1) and 2). But they make no mention of 3). Digitality flavor 3) is needed for the brain not only to draw inferences but also to act upon those inferences.

From a slightly different perspective, 2) can be subdivided in the three digital operations Supplementation (S), Partition (P), and Abolition (A). If one adds to these three the digital operation certification of digitality flavor 3 ), then it appears that the core of logical reasoning that leads to action consists of four digital operations S, P, A, and $\mathrm{C}$, which come in that order.

Digitality flavor 4) pertains less directly to logical reasoning. But it confirms the profoundly digital structure of rational language and thought. It completes the picture, as it were.

\section{Rational Intelligence and Rational Knowledge}

There is more to rationally intelligent human behavior than rational human intelligence. For example, it is not necessary to know that acceleration is change in velocity over time to be endowed with rational human intelli- 
gence. It is not necessary to know the laws of gravitation to be endowed with rational human intelligence. After all, how could it be? If that were the case, the vast majority of the earth's population would lack rational human intelligence. And yet, just about all of earth's population is endowed with rational human intelligence.

Then how does a statement like "Acceleration is change in velocity over time" evidence rational human intelligence? The statement associates exactly two things or entities identified by their attributes with one another, not three or more. In that regard, rational human intelligence is fundamentally binary.

The statement relates to rational human intelligence strictly speaking is as follows. It unambiguously conveys that there is neither something that is at the same time acceleration and not change in speed over time nor something that is at the same time change in speed over time and not acceleration. Two things are declared to be non-existent in said statement.

The statement involves two attributes of things. One attribute is signified by the noun "acceleration". The other attribute is signified by the noun phrase "change in velocity over time". Each of the two things that are declared to be non-existent is digitally defined in terms of attributes that it does or does not exhibit. One thing exhibits the attribute "acceleration" but not the attribute "change in speed over time". The other thing exhibits the attribute "change in speed over time" but not "acceleration". Again, the statement conveys that neither exists. That part of the statement is an expression of rational human intelligence.

If the laws of gravity cannot be part of rational human intelligence, then what are they part of? They are not part of intelligence. Rather, they are part of knowledge.

The relation between intelligence and knowledge can be clarified by analogy with an electric motor. Intelligence is like the operation of the motor. Knowledge is like the tasks performed by the electric motor.

It follows that all quantitative mathematics, that is, the familiar mathematics (or that which everyone would usually understand to be mathematics), is part of knowledge. None of it is part of rational human intelligence. Rational human intelligence is in its entirety subject only to the laws of non-quantitative, digital mathematics.

\section{The Reflexivity of Rational Human Intelligence}

Not all non-quantitative digital mathematics pertains to rational human intelligence. In other words, some of it does and some of it does not. However, the nature of rational human intelligence is such that the part of digital mathematics that does relate to rational human intelligence can also be part of knowledge in addition to being part of intelligence. Rational human intelligence as a brain function can be applied to all of physical reality. And that physical reality includes the functions of the brain that relate to rational human intelligence.

There is only one way for the brain to analyze rational human intelligence, and that is by means of rational human intelligence itself. The brain is a tool that can observe itself functioning and analyze that function. This property may be called reflexivity.

G. Boole evokes this reflexivity in a more general way as follows [13]:

Even in ages the most devoted to material interests, some portion of the current of thought has been reflected inwards, and the desire to comprehend that by which all else is comprehended has only been baffled in order to be renewed.

In this regard, the analogy between the brain as a tool and an electric motor as a tool breaks down. The electric motor can move itself by being attached to wheels. But the energy that it produces cannot be put in the service of the very energy that it produces.

\section{Syllogisms as a Component of Digital Mathematics}

\subsection{Digital Mathematics That Is Part of Rational Human Intelligence and Digital Mathemathics That Is Not}

It will be good to positively ascertain by means of an illustration that there is non-quantitative, digital mathematics outside of the non-quantitative mathematics that explains rational human intelligence. The digital mathematics that falls outside rational human intelligence is of interest in the present context precisely because of its affinity with the mathematics that pertains to the operation of rational human intelligence. There is in fact a certain potential for this other digital mathematics to be confused with rational human intelligence by being considered part of it, and in fact it has been. It is therefore of particular importance to distinguish the digital mathematics that is part of rational human intelligence from the digital mathematics that is not part of rational human intelligence. In that regard, it will be useful to assess and evaluate the latter to better demarcate and understand the former.

\subsection{A Syllogism Proposed by Ch. L. Dodgson}

The focus of what follows will be on an example of a matter that requires digital mathematics other than the digital mathematics involved in the operation of rational human intelligence. The example in question is the following derivation found in part I of the well-known book on symbolic logic by Ch. L. Dodgson.

Like his literary work, which includes Alice in Wonderland, Ch. L. Dodgson published his work on logic 
under the pseudonym of Lewis Carroll. It is not clear what one is to infer from this about his work on logic. As is well-known, his literary work is full of logical riddles. It has been appreciated by a very large audience for several generations now. But still, this facet of his work is unusual and, one might even say, eccentric. I would definitely classify his work on logic as very earnest and very clever in many ways but also idiosyncratic in some others. One even wonders whether some of it is not tongue in cheek.

In the example in question, it is asked what can be derived from the following two statements [14]:

All philosophers are logical;

An illogical man is always obstinate.

Ch. L. Dodgson's conclusion is:

Some obstinate persons are not philosophers.

It seems clear that, by "man", people in general are meant.

The example came to my attention because it is cited in a book by $\mathrm{Ch}$. Petzold that explains in exemplary fashion how computers are built from scratch. I have not found anything quite like it anywhere.

Like the rational intelligence of the brain, the machine intelligence of a computer is digital. However, at this time, the digital operations pertaining to rational human intelligence are more diverse than those pertaining to machine intelligence. Both share the Boolean operators AND, OR, and NOT. But rational human intelligence exhibits additional digital functions. What sets apart the computer from the brain is not complexity or diversity but (1) size and (2) speed, which both can be colossal when compared to rational human intelligence.

$\mathrm{Ch}$. Petzold comments as follows on the example taken from Ch. L. Dodgson [15]:

The conclusion isn't obvious at all. (It's "Some obstinate persons are not philosophers". Notice the unexpected and disturbing appearance of the word "some".)

This arrangement in which a conclusion is drawn from two premises is evidently the syllogism. Perhaps the best known example, also cited by Ch. Petzold, is the following:

All men are mortal;

Socrates is a man;

Hence, Socrates is mortal.

\subsection{Syllogisms as Extraneous to Rational Human Intelligence}

The first observation to be made is that the analysis of the two syllogisms presented in $\S 13.2$ and of any syllogisms for that matter is not part of rational human intelligence. Plainly put, it is possible to completely fail to understand how conclusions are derived from premises in syllogisms and yet to be fully endowed with rational human intelligence. Most people know very little or nothing about syllogisms and yet exhibit rational human intelligence.

Still, a proper understanding of the derivations requires the same type of mathematics by which rational human intelligence operates. Attention to the mathematical analysis of the derivations may therefore shed an indirect light on the nature of rational human intelligence. It only adds to the interest of the derivations that they are mentioned in a book on machine intelligence, which also operates according to digital mathematics.

\subsection{The Dual Nature of G. Boole's Digital Mathematics}

All that follows in the present paper is so squarely based on what G. Boole and his principal expositor J. Venn teach that one way in which the following account significantly deviates from theirs needs to be pointed out.

There is no doubt from the organization of his work that G. Boole considered all digital mathematics to be of one and the same species. And in a sense it is. It is all non-quantitative. G. Boole presents a complete account of the digital mathematics that pertains to attributivity, that is, to the property of things and events to be characterized by attributes in both their present state and their absent state.

But nowhere does G. Boole intimate that some of the digital mathematics pertaining to attributivity pertains to rational human intelligence and some of it does not. G. Boole therefore does two distinct things in his work on digital mathematics. First, he describes in part the rational operations of the mind, which are digital. Second, he does digital mathematics.

When doing digital mathematics, G. Boole does nothing different from doing non-digital mathematics such as trigonometry or calculus. No one would state that calculus is part of the structure of rational human intelligence. It is part of acquired knowledge. Thus, G. Boole's fundamental formula of Development,

$$
f(x)=f(1) x+f(0)(1-x),
$$

is perfectly digital, just like rational human intelligence. It can be studied and understood by the digitally thinking brain. But there is no way that this formula is part of rational human intelligence. If it were part of rational human intelligence, then the rational human intelligence of anyone who is not aware of this formula would in some way be deficient. It is not.

A main reason that G. Boole conflates-and one might perhaps add, confuses - the digital mathematics that explains rational human intelligence and the digital mathematics that lies outside rational human intelligence is that both are digital. But there is another important reason that makes it very tempting to conflate the two.

The only way in which the brain becomes consciously 
aware of the digital mathematics by which its rational intelligence operates is by describing it. However, by describing it, the brain is actually doing digital mathematics. To the extent that it is studied by the brain, rational human intelligence is not a function of the brain anymore. It is something to which the rational intelligent function of the brain is applied. Rational human intelligence is in a sense subjected to itself. In that regard, when the brain describes the digital mathematics that pertains to rational human intelligence, it is not doing anything different than when it describes the digital mathematics that does not pertain to rational human intelligence. It is just all digital mathematics.

This sameness may obscure the distinctness and discreteness of the digital mathematics that pertains specifically to rational human intelligence from the digital mathematics that does not. The design of what is to come in this and other papers is to delineate precisely the type of digital mathematics that pertains specifically to rational human intelligence.

The phenomenon at hand has already been called reflexivity in $\S 12$ above. The brain uses the digital mathematics by which it operates to describe the digital mathematics by which it operates.

I have elsewhere described a somewhat similar type of reflexivity pertaining to linguistic expression. It is possible to speak and at the same time to speak about speaking [16]. In the same way, it is possible to reason about rational human intelligence while using rational human intelligence. An example in which both co-occur would be the statement

" "Socrates is mortal' means exactly that nothing is both Socrates and not mortal'.

This statement is about a derivation involving a type of digital mathematics that does not define rational human intelligence. Then what part of the above statement reflects rational human intelligence? It is the part that conveys that there is no such thing that is the statement "Socrates is mortal" and at the same time does not mean that nothing is both Socrates and not mortal. But again, understanding how the derivation is made is not part of the structure of rational human intelligence but rather an application of rational human intelligence.

The above distinction may be subtle. But I believe it to be crucial. By not making the distinction, G. Boole may well have confused his readers as to what he was trying to do. Some have believed that he was trying to explain how people think rationally. Others have believed that he was doing mathematics. He was in fact doing both in the same book without explicitly distinguishing between the two. The two endeavors are intimately related because the mathematics that explains rational human intelligence is digital and the mathematics beyond rational human intelligence that $\mathrm{G}$. Boole does in the book is also digital.
What is more, rational human intelligence needs to be explained by means of rational human intelligence itself. Nothing is therefore easier than confusing the two.

Because of the lack of a distinction, it is more than tempting to assume that $\mathrm{G}$. Boole somehow considers syllogisms of the type described above to be part of rational human intelligence. G. Boole does after all analyze them in his book entitled "An Investigation of the Laws of Thought" [17]. But they are not rational human intelligence. Still, a full and final understanding of syllogisms requires digital mathematics and that is also the mathematics of rational human intelligence. Syllogisms can therefore easily be seen as a kind of extension of rational human intelligence that trigonometry or linear algebra are not. Furthermore, the mathematics that the brain uses to analyze syllogisms is of the same type as the mathematics of which rational human intelligence is constituted.

\subsection{Syllogisms as Pure Mathematics}

In order to illustrate the digital mathematics that falls outside rational human intelligence, it will be good to explicitly analyze the two syllogisms cited above in $\S 13.2$ in strictly mathematical terms.

The possibility of a complete mathematical analysis serves as testimony to the effectiveness of rational human intelligence. The analysis of syllogisms is usually considered part of a field called logic. However, I expressly refrain from calling the following analysis logical. Calling what follows logic is in danger of suggesting that what follows is in some sense not $100 \%$ mathematical. And yet, it is.

Calling the analysis logical is also in danger of suggesting that there may be more than one way of analyzing the syllogism. And yet, there is not.

Everyone accepts that there is only one mathematics. There are no circles in which the ratio between the diameter and the circumference of a circle is not $\pi$. But one has the impression from looking at works on logic that there is a sense out there that there may be more than one way of doing logic.

There is no doubt whatsoever that logic has been represented over the decades in many different formal expressions. As far as I am concerned, this is exactly the same as representing the same mathematical concepts with different symbols. To be sure, there is some of that in mathematics too. For example, the derivative of differential calculus is presented symbolically in quite a few ways. But in mathematics, there is no doubt that different symbolic representations signify exactly the same.

It was noted above that $\mathrm{Ch}$. Petzold observes that

Some obstinate persons are not philosophers

is "not obvious" as a conclusion from the two premises

All philosophers are logical 
and

An illogical man is always obstinate [18].

One of the reasons that the conclusion is "not obvious" is that it is wrong. Furthermore, being wrong is not the only deficiency of the conclusion. The conclusion leaves much unsaid about what exactly can and cannot be derived from the two premises.

What can exactly be inferred from the two syllogisms? On the page following the one on which he mentions the two syllogisms, Ch. Petzold prominently refers to G. Boole as a founding figure or modern computer science ("And then came Boole") [19].

Now it appears that G. Boole had already furnished all the tools needed to analyze the two syllogisms mentioned on the previous page of Ch. Petzold's book to full satisfaction in strictly mathematical terms. The details are presented below. Yet, there is no mention of this facet of G. Boole's work in Ch. Petzold's book. This may serve as an illustration of my belief that, whereas G. Boole's seminal contribution to the digital mathematics of computer code is fully acknowledged, it seems little realized that his contribution to the digital mathematics of rational human intelligence may well be of similar importance.

In fact, Ch. Petzold's evaluation of what G. Boole has to say about rational thought and language is hardly enthusiastic. But it does end on an intriguing statement placed between parentheses [20]:

The title of Boole's 1854 book suggests an ambitious motivation: Because the rational human brain uses logic to think, if we were to find a way in which logic can be represented by mathematics, we would also have a mathematical description of how the brain works. Of course, nowadays this view of the mind seems to us quite naive. (Either that or it's way ahead of its time.)

I personally discern in the final words a whiff of clairvoyance. I hope to demonstrate further below the option left open by Ch. Petzold, namely that "it's way ahead of its time", by means of the seamless resolution of the two syllogisms mentioned both by Ch. Petzold and also in $\$ 13.2$ above in their broader context and then in additional papers by means of presenting a full map of rational human intelligence.

The mathematical analysis of the two syllogisms to be presented below is based squarely on G. Boole's [21]. G. Boole calls a derivation of a conclusion from two premises a reduction.

The analysis is strictly speaking not relevant to the main line of argument of the present paper and future papers, which is the description of rational human intelligence. It can therefore be skipped. Then again, it would have been unsatisfying to just impress on readers that the analysis of syllogisms is possible in purely mathematical fashion and rely on their good faith to accept that this is so. Some explicit illustrations are needed to drive the point home. These illustrations offer an opportunity to positively and independently verify the strictly mathematical nature of the derivations. Again, the following analysis of syllogisms is not something separate from mathematics that requires a distinct name such as logic. It is pure mathematics.

\subsection{The Larger Mathematical Problem of Which the Syllogism Is Part}

Before proceeding to the mathematical analysis of the syllogism, it needs to be noted that the syllogism is just one special case of a much larger mathematical problem, as G. Boole was the first to observe clearly and in detail. To be sure, there is nothing wrong with Aristotle's syllogisms. They altogether lead to universally valid conclusions. But they offer only a very partial view of what conclusions can be drawn from two or more propositions.

When it comes the larger problem, G. Boole was not only the first to fully appreciate it. He also solved it more or less completely. In that regard, it is interesting to read the following evaluation of J. Venn, G. Boole's principal expositor [22]:

The general solution of this problem was probably first conceived, and almost certainly first effected, by Boole. As a piece of formal symbolic reasoning there seems nothing to be added to it as he left it, and it is a striking example of his penetration and power of generalization. It cannot often be the lot of any one to conceive and so completely to carry out such a generalization in an old and well-studied subject.

G. Boole's work was available when Ch. L. Dodgson wrote his book on logic. And Ch. L. Dodgson does seem to have followed the work of his contemporaries and his antecedents to some degree, including G. Boole's [23]. But he evidently made no use of G. Boole's analysis. It appears to me that G. Boole's analysis suspends all need for Ch. L. Dodgson's.

What is the larger mathematical problem of which the syllogism is part and how does the smaller mathematical problem of the syllogism relate to it? It will be useful to take one of the two syllogisms mentioned in \$13.2, namely Ch. L. Dodgson's, and see how it can be expanded into a much larger mathematical problem.

The properties of the syllogism are rather specific, in four regards.

First, there are two premises and one conclusion, as in all syllogisms.

Second, there are three attributes, as in all syllogisms. In this case, the three attributes are "philosopher", "logical", and "obstinate".

Third, as in all syllogisms, the ways in which the attributes relate to one another in the premises and the conclusion are as follows. 
The first premise, "All philosophers are logical", relates the first attribute "philosopher" to the second "logical". The second premise, "An illogical man is always obstinate", relates the second attribute "(il)logical" to the third "obstinate". Accordingly, the second attribute appears twice in the premises. Finally, the conclusion is designed to establish what can be derived about the relation between the first attribute and the third attribute from the two premises.

Fourth, the attributes exhibit certain manifestations of their present and absent states. It is characteristic of the syllogism under examination that the attribute that appears twice, namely the second, appears once in the present state, "logical", and once in the absent state, "illogical". Such a configuration is not part of the classical Aristotelian and medieval system of the syllogism. In some sense, not everyone would call Ch. L. Dodgson's derivation a syllogism for that reason.

The full expansion of the syllogism into a larger mathematical problem is as follows.

The first expansion is to any number of premises from two premises and to more than one conclusion, as many conclusions as are possible. The second expansion is to any number of attributes from three attributes. The third expansion is to any way of relating attributes to another. The fourth expansion is to any configuration of present and absent states.

For example, there might be 12 premises with 14 attributes and several conclusions.

There is one characteristic, however, that cannot be expanded. Each premise and each conclusion is an equation between exactly two items. Rational human intelligence is profoundly and totally nexal. Nexus is Latin for "link". A nexus links two and exactly two concepts, as in $x=y$. Accordingly, an Equation such as $x=y=z$ cannot be comprehended unless it is treated as $x=y$ and $y=z$.

Deriving conclusions from premises requires digital mathematics. Rational human intelligence is digital. But the digital mathematics under discussion presently is not itself rational human intelligence. It is something to which rational human intelligence is applied. And the object of application happens to be just as digital as rational human intelligence. As has already been noted above, it is easy to confuse the two, as G. Boole in fact did.

\section{The Mathematical Analysis of All Philosophers Are Logical, an Illogical Man Is Always Obstinate and Therefore: Some Obstinate Persons Are Not Philosophers}

\subsection{Theoretical Foundations}

G. Boole's definitive mathematical analysis of the syllogism contains three principal operations: Expansion,
Elimination, and Reduction. All three operations will be applied below. But it will not be possible to present the complete theoretical foundations underlying these interpretations. In that regard, I refer to G. Boole's foundational "Investigation of the Laws of Thought" [24] and J. Venn's useful and lucid clarifications of G. Boole's theory [25].

There are quite a few accounts of G. Boole's algebra. But these accounts as a rule mix G. Boole's algebra with other systems of notation of what is called logic or the like and there are many of them. I have personally never seen any reason to depart in any significant way from $G$. Boole's notation. In my opinion, it provides all the power and the perfect efficiency that one needs in order to describe rational human intelligence to its full extent. I am not sure to which extent a certain desire for originality has fueled the genesis of new notations. If anything, my impression is that alternative notations diminish the potential for describing rational human intelligence to its full extent.

One reads on occasion that G. Boole tried to describe how we think rationally and failed. One of the designs of the present paper and follow-up papers is to demonstrate that G. Boole took us most of the way towards that goal.

\subsection{The Three Components of a Complete Mathematical Analysis}

The properties of the syllogism have already been detailed above. Quite in general, the purpose of the syllogism is twofold. The first aim is to reduce or compact two premises into one conclusion. The second aim is to eliminate an attribute.

The evaluation of Ch. L. Dodgson's syllogism will involve three tasks. In all three tasks, the evaluation of the second syllogism cited in $\$ 13.2$ above will be used as a control mechanism.

The first task (see $\S 14.4$ and $\S 14.5$ ) is to establish that the conclusion cannot be derived from the two premises. In fact, no conclusion pertaining to the relation between the first and the third attribute can be derived from the premises.

The second task (see $\$ 14.6$ ) is to establish what Ch. L. Dodgson's conclusion exactly means. It cannot be denied that the conclusion does have a purport. But the purport in question cannot possibly follow from the premises. Nor in fact can it follow from any other possible premises.

The third task ( $§ 14.7$ and $\S 14.11)$ is to establish what conclusions do follow from the premises. There are indeed some.

\subsection{Mathematical Notation}

The mathematical notation that will be used in the pre- 
sent analysis of the syllogism and in the description of rational human intelligence in this paper and other papers to follow will be as much as possible G. Boole's own. I am otherwise aware that there are many systems of notation in existence in logic. As I have already intimated above, I personally find G. Boole's to be superior to all.

G. Boole's is also as close as possible to the notation of classical quantitative mathematics, the familiar mathematics. The symbols do not necessarily have the same signification as in quantitative mathematics. But even when they do not, they can be similar in terms of signification.

I believe with J. Venn that, in taking advantage of the similarity and avoiding an entirely new set of symbols, the benefits decisively outweigh the drawbacks. I will return to this point further below.

Hence, let $p$ be "philosopher". Let $l$ be "logical". And let $o$ be "obstinate".

Accordingly, 1-l denotes "non-logical, illogical", that is, the universe of thought (G. Boole's "1"), or all that one could possibly think about, minus anything that is $l$. G. Boole often abbreviated $1-l$ to $\bar{l}$, as did J. Venn in his footsteps. This convention will be followed off and on below.

The two premises of the syllogism at hand can now be formulated as follows:

$$
\begin{aligned}
& p=p l \text { "All philosophers are logical", } \\
& \text { or also } p=p \times l ; \\
& 1-l=(1-l) o \\
& \text { "An illogical man is always obstinate", } \\
& \text { or also } 1-l=(1-l) \times o \\
& \text { and } \bar{l}=\bar{l} \times o .
\end{aligned}
$$

As the symbolic equivalent of "All philosophers are logical", the notation $p=p l$ will be used in the present paper and future papers on the nature of rational human intelligence. It is also the notation preferred by J. Venn. However, G. Boole himself normally does not use it. It is one of the few ways in which my notation will differ from G. Boole's. Then again, J. Venn points out that, while the notation in question is "not primarily employed by Boole", it "is constantly presenting itself in the course of [G. Boole's] analytical processes" [26].

Remarkably, as J. Venn notes, G. W. Leibniz already used a variation of this notation. J. Venn quotes the following from G. W. Leibniz's essay entitled "Difficultates Quaedam Logicae" ("Certain Difficulties in Logic") [27,28]:

Omne $A$ est B; i.e. aequivalent $A B$ et $A$ ("All $A$ is $B$; that is, $A B$ and $A$ are equal').

In the same essay, G. W. Leibniz also describes the same relation as follows [29]:

Omne $A$ est B, i.e. AB aequivalet ipsi $A$ ("All $A$ is $B$; that is, $A B$ is equal to $A$ ").

And a little further, he writes [30]:

Omnis ridens est homo i.e. ridens et ridens homo aequivalent ("All laughers are human; that is, laughers and laughing human beings are equal").

In this connection, there has been much discussion about G. W. Leibniz's place in the history of mathematical logic. There is no doubt that his works on logic are full of interesting insights and J. Venn, who was an outstanding student of the history of logic, points out many of these insights in his own work. It would also appear that G. W. Leibniz and even considerably more the now completely neglected J. H. Lambert [31] were the two most astute students of mathematical logic before G. Boole.

About J. H. Lambert, J. Venn goes as far as stating the following [32]:

$[I] f[G$. Boole $]$ had knowingly built upon the foundation laid by his predecessor [J. H. Lambert], instead of beginning anew for himself, it would be hard to say which of the two had actually done the most.

However, it is not possible to find in the works of either G. W. Leibniz or J. H. Lambert anything close to the comprehensive theory of digital mathematics that is evident in G. Boole's works.

This is otherwise not the place to discuss the respective merits of the various forms of notation. Still, the one preferred here, $p=p l$, offers distinct advantages. One advantage is the manner in which it conveys in transparent fashion the purport of the statement that it represents symbolically.

The purport of the notation is easily enough put into words. The Equation clearly signifies that the set of philosophers $(p)$ is the same as the set of anything that is both a philosopher and logical $(p \times l$ or $p l)$. This means that the set of the philosophers is a subset of that which is logical. Indeed, if the philosophers are all logical, then thinking of all the philosophers is the same as thinking of all the logical philosophers because they are all logical.

Even more convenient is a geometric representation. The set of the philosophers may be represented by a smaller circle inhabiting a larger circle representing the set of all that is logical. Evidently, the smaller circle of the philosophers belongs to the larger circle of what is logical and therefore contains all that is both a philosopher and logical. On the other hand, there can be logical entities outside the set of the philosophers.

From Equation (1), it follows that

$$
p-p l=0,
$$

which can also be written as

$$
p(1-l)=0 .
$$

Equation (3) can be put into words as 
"Nothing (0) is $(=)$ both a philosopher $(p)$ and not logical $(1-l)$ ".

That $p(1-l)$ equals $(=)$ nothing $(0)$ means that it does not exist. Likewise, it can be inferred from the second premise, Equation (2), that

$$
(1-l)-(1-l) o=0
$$

which can evidently be rewritten as

$$
(1-l)(1-o)=0
$$

and be put into words as

"There is no such thing as something that is both illogical and not obstinate".

There appears to be more than one way to accomplish mathematical derivations in G. Boole's algebra. "It is, however, satisfactory", writes G. Boole, "to possess different modes, serving for mutual verification, of arriving at the same conclusion" [33].

\subsection{Task One: Establishing That the Conclusion Does Not Follow from the Premises}

Do the two premises lead to the conclusion in the syllogism at hand? It appears they do not. In fact, they afford no conclusion whatsoever about the relation between "obstinate" $(o)$ and "philosopher" $(p)$. The procedure that G. Boole would follow is as follows [34].

First, Equations (3) and (4) are combined and reduced to a single Equation, as follows:

$$
p(1-l)+(1-l)(1-o)=0
$$

Since the Equations (3) and (4) both involve a term that is equated with 0 , their sum in (5) will also be equal to 0 .

Second, in order to eliminate $l$ to leave only $p$ and $o$ and thus establish how $p$ and $o$ relate to one another, G. Boole's mathematically proven procedure contains the following three steps: 1) equating $l$ in the left term of Equation (5) first with 1 and then with 0;2) multiplying the two results; and 3) equating the product with 0 [35]. Equating $l$ in the left term of (5) with 1 gives

$$
\begin{aligned}
& p(1-1)+(1-1)(1-o) \\
& =p \times 0+0 \times(1-0) \\
& =0+0 \\
& =0
\end{aligned}
$$

Equating $l$ in the left term of Equation (5) with 0 gives

$$
\begin{aligned}
& p(1-0)+(1-0)(1-o) \\
& =p \times 1+1 \times(1-o) \\
& =p+(1-0)
\end{aligned}
$$

The result of multiplying (6) and (7) and equating the product with 0 is

$$
0 \times[p+(1-0)]=0
$$

And therefore also

$$
0=0 .
$$

This expression is completely inconclusive. It follows that there is nothing to be learned from the two premises about the relation between $p$ and $o$. QED.

It will be useful to verify this conclusion by applying the same procedure to the other syllogism cited in $§ 13.2$. In the case of this well-known syllogism, there is no one who doubts that the conclusion follows from the two premises. It will therefore be interesting to see how this other syllogism fares when it is subjected to the exact same procedure as Ch. L. Dodgson's syllogism.

The two premises are as follows:

All men are mortal;

Socrates is a man.

If "men" is $h$ (that is, "human"), "mortal" is $m$, and "Socrates" is $s$, then the two premises can be represented in complete analogy with the two premises of Ch. L. Dodgson's syllogism as follows:

It follows that

$$
\begin{gathered}
h=h m ; \\
s=s h .
\end{gathered}
$$

$$
\begin{gathered}
h-h m=0 \text { or } h(1-m)=0 \text { and } \\
s-s h=0 \text { or } s(1-h)=0 .
\end{gathered}
$$

Adding the left terms of the two Equations and equating them with 0, as was done in Equation (5), produces

$$
h(1-m)+s(1-h)=0 .
$$

The common term, in this case $h$ "human", can be eliminated in the same way that it has been above in $\mathrm{Ch}$. L. Dodgson's syllogism. In this case, the common term is $h$ "human". Its elimination makes it possible to establish what the relation is between $s$ and $m$.

The attribute $h$ in the left term of the Equation is first equated with 1 and then with 0 . The two results are multiplied with one another, and the product is equated with 0 .

Equating $h$ in the left term of (8) with 1 gives

$$
1 \times(1-m)+s(1-1)=1-m .
$$

Equating $l$ in the left term of (8) with 0 gives

$$
0 \times(1-m)+s(1-0)=s
$$

The result of multiplying (9) and (10) and equating it with 0 is

$$
s(1-m)=0 .
$$

This can also be written as 


$$
s-s m=0 .
$$

And therefore also as

$$
s=s m .
$$

This last Equation can be put into words as "Socrates is mortal". Indeed, according to this Equation, conceiving of Socrates $(s)$ is exactly the same as conceiving of all that is both Socrates and mortal $(\mathrm{sm})$. The same entities are selected. In other words, there is nothing that is Socrates beyond what is mortal. Conversely, conceiving of mortals $(\mathrm{m})$ is not the same as conceiving of all that is both Socrates and mortal $(\mathrm{ms})$. There are more of the former than of the latter. There are mortals outside of Socrates, but no Socrates outside of mortals. In sum, Socrates belongs to the mortals.

It appears, then, that the premises of the second syllogism, as contrasted with those of Ch. L. Dodgson's syllogism, do afford a conclusion.

Ch. L. Dodgson's syllogism is not the only type that offers no mathematical conclusion. So, for example, does the following type:

No a is b.

No b is c.

It is not possible to draw a conclusion from these two premises about the relation between $a$ and $c$ : $a$ may be 1) none of $c$ or 2) some of $c$ or 3) all of $c$ (and perhaps then some). One example of each is as follows:

1) Brazilians are not French.

French are not Germans.

2) Brazilians are not French.

French are not South Americans.

3) Brazilians are not French.

French are not citizens of Rio de Janeiro.

In the case of 1), it is otherwise known that $a$ ("Brazilians") are none of $c$ ("Germans"); in the case of 2), that $a$ is some of $c$ ("South Americans"); and in the case of 3), that $a$ is all of $c$ ("citizens of Rio de Janeiro") and then some.

\subsection{Interlude: The Real Life Purport of the Inconclusiveness of $\mathrm{Ch}$. L. Dodgson's Two Premises}

It was noted above that the mathematical conclusion derived from Ch. L. Dodgson's two premises regarding the relation between the two attributes $p$ "philosophers" and $o$ "obstinate people" in both their present and absent states can be expressed as

$$
0=0 \text {. }
$$

In other words, the two premises yield no conclusion regarding the relation between $p$ and $o$. Nothing can be known about the relation between the two on the basis of the two premises.

The mathematical conclusion may seem a little ab- stract and may therefore fail to convince. It will therefore be useful to give some real life perspective to the mathematical conclusion by means of concrete examples.

A number of relations are possible between $p$ and $o$. This is not the place for a detailed account. A complete discussion of all the possible relations between two attributes in their present and absent states needs to be postponed to the detailed treatment of nexus digitality (NexDi).

But in brief, the main types of relations between $p$ and $o$ may be expressed as follows:

1) "All $p$ is $o$ ";

2) "All $o$ is $p$ ";

3) "All $p$ is all $o$ ", which is the same as "All $o$ is all $p$ ", implying both "All $p$ is $o$ " and "All $o$ is $p$ "; and

4) "No $p$ is $o$ ", which is the same as "No $o$ is $p$ ".

Instead of discussing all the possible relations between $p$ and $o$, it will be advisable to instead take Ch. L. Dodgson's conclusion as a point of departure and determine how it compares to the strictly digital mathematical conclusion.

According to Ch. L. Dodgson's conclusion, some obstinate people are not philosophers. It may be assumed that, by "some", Ch. L. Dodgson means at the same time both "not all" and "not none". In his definition of "some" as "one or more" [36], "more" might perhaps be interpreted as including "all" because "all" is after all "more than one". However, he also classifies "some", "none", and "all" as being all three distinct from one another [37]. Furthermore, he classifies "some" as part of a "particular" proposition and "all" as part of a "universal" proposition [38], as is the rule in Aristotelian and scholastic logic. In other words, if "some" included "all", "some" could be on occasion be part of a universal proposition, which according to Ch. L. Dodgson is never the case.

In order to disprove Ch. L. Dodgson's conclusion in a concrete way, it needs to be demonstrated by means of clear real life examples that the two conclusions "All obstinate persons are non-philosophers" and "No obstinate persons are non-philosophers" are altogether possible.

It may be argued that the fact that these two other conclusions are also possible does not make Ch. L. Dodgson's conclusion false, only incomplete. However, at issue is mathematics. A mathematical conclusion needs to seamlessly account for all possible cases and describe the exact extent both of what is known and what is not known. When it comes to mathematics, an incomplete answer is a false answer.

Moreover, even though "some" is not quite contradicted by "all", it definitely is by "none".

First, "all" in addition to "some". There seems to be no reason why one of the possible conclusions could not be that all the obstinate people are non-philosophers, and 
not just some as Ch. L. Dodgson concludes, in other words that no obstinate people are philosophers. Ch. L. Dodgson's conclusion "Some obstinate persons are not philosophers" seems to imply that some obstinate persons are philosophers. But this implication, while possible, is not binding. Again, mathematically speaking, the possibility cannot be excluded that no obstinate persons are philosophers.

Even if "some" did mean "at least some" and hence did include "all" according to Ch. L. Dodgson, which I believe it does not (see above), the expression "some" would be mathematically imprecise.

The matter can be clarified and visualized by means of circles representing sets or classes. It may be useful for the reader to actually draw these circles. If a circle $P$ inhabits a circle $L$, signifying that philosophers $(P)$ are members of the class of logical persons $(L)$ in accordance with Ch. L. Dodgson's first premise, then illogical persons are represented by the entire area that falls outside the larger circle $L$.

According to Ch. L. Dodgson's second premise, what is outside circle $L$ is part of what is obstinate. Now imagine a circle $O$ signifying what is obstinate and enveloping all that is outside $L$. According to Ch. L. Dodgson's conclusion, circle $O$ also seems to envelop part of $P$. In other words, some obstinate persons are philosophers.

It seems clear that, for circle $O$ to envelop all that is outside $L$, it does not necessarily in addition need to encompass anything inside circle $L$ or anything inside the circle $P$ that is inside circle $L$. If it does neither, then all obstinate persons are both at the same time illogical and non-philosophers. It then also follows that $O$ and what is outside $L$ fully overlap. In other words, all obstinate persons are non-philosophers and all non-philosophers are obstinate persons. The two are the same.

One might argue that it is somehow more plausible that some philosophers are obstinate and some are not, as Ch. L. Dodgson's conclusion implies, rather than that all obstinate persons are non-philosophers, a possibility that cannot be excluded according to a strictly mathematical analysis. There is no denying that Ch. L. Dodgson's conclusion seems somehow more plausible. But the simple point is this. This plausibility is derived from what else is known about philosophers and obstinate persons. It cannot be derived from the mathematical structure of the two premises.

In this regard, it will be useful to consider examples of pairs of premises that exhibit the exact same structure as Ch. L. Dodgson's pair, but in which the conclusion "All $z$ is not $x$ " seems more plausible than Ch. L. Dodgson's conclusion "Some $z$ is not $x$ ". His pair is juxtaposed to the new pairs to reveal the identity in structure.

First premise:
All philosophers are logical.

All French people are human beings.

Second premise:

An illogical man is always obstinate.

A non-human being is always devoid of rationality.

Conclusion:

Some obstinate persons are not philosophers.

Some devoid of rationality are not French persons.

The conclusion leaves open the possibility that some devoid of rationality are French persons. It is desirable and tempting, to say the least, to assume instead that all those devoid of rationality are not French persons, or that no one devoid of rationality is French.

In the case of "obstinate" in Ch. L. Dodgson's syllogism, the possibility that some philosophers are obstinate is not far-fetched. It is not all that difficult to imagine obstinate philosophers. But it does not come so easy to imagine French people devoid of all rationality. If an attribute is undesirable, like being devoid of rationality, it is much easier to assume or want to assume that no member of a certain class or set exhibits it. This assumption also comes easy in the case of the following two premises.

According to Ch. L. Dodgson's pattern, the two premises

All brilliant scientists are human beings.

A non-human being is always devoid of rationality. lead to the following conclusion:

Some devoid of rationality are not brilliant scientists.

Surely, all of them are not brilliant scientists, that is, none of them are brilliant scientists.

It cannot quite be said that the conclusions regarding those devoid of rationality are false. But they are incomplete in leaving out a distinct possibility. In that regard, they are not mathematical. Once the possibility left out is included, the two premises become less conclusive than Ch. L. Dodgson makes them out to be.

There are some pairs of premises modeled after Ch. L. Dodgson's pair of premises in which the conclusion would seem to require "all" at the exclusion of "some". That would in a sense in its own right be a rejection of "some" as Ch. L. Dodgson's conclusion. While assuming "all or some" instead of just "some" just makes Ch. L. Dodgson's conclusion incomplete, just "all" would contradict it.

Consider in this regard the following two premises and a conclusion modeled after Ch. L. Dodgson's syllogism:

Belgian cyclists are drug free.

A doper (someone non-drug-free) is always banned.

Therefore, some banned persons are not Belgian cyclists.

It would seem as if the conclusion that all banned persons are not Belgian cyclists, in other words that no banned persons are Belgian cyclists, imposes itself. After 
all, they are all drug free. Why ban them?

However, the premises only state that dopers are banned. They do not reveal whether drug free cyclists are banned or not. The assumption that they are not banned is not derived from the premises but from what else is known outside the premises. Considering all else that is known about cyclists, it is indeed difficult to think of a reason why drug free cyclists would be banned. But in drawing this conclusion, one relies on what else one knows besides the premises. Theoretically speaking, it is easy to think of a possible world in which cyclists, including drug free ones, can be banned for various types of behavior besides doping. It is not possible to exclude this possibility.

This much for "all". But what about "none"? So far, "all" has been added to Ch. L. Dodgson's "some" as a possible conclusion to show that what can be inferred from the premises is less conclusive than Ch. L. Dodgson assumes. But since "all" in a sense automatically implies "(at least) some" whereas "some" does not automatically imply "all", it is possible to argue that Ch. L. Dodgson's conclusion is not positively falsified but only exposed as incomplete and therefore mathematically imprecise.

In order to expose the two premises as fully inconclusive and at the same time positively false, it needs to be established whether "none" is also a possible conclusion, in this case that "None of the obstinate persons are not philosophers", or in other words that "All the obstinate persons are philosophers".

The implications of this possible conclusion are as follows. According to the first premise, all the philosophers are logical. If one in addition assumes, as was just done on the basis of "None of the obstinate persons are not philosophers", that all the obstinate persons are philosophers, then by the first premise the obstinate persons are also all logical.

However, according to the second premise, an illogical person is always obstinate. Accordingly, one is forced to contemplate the notion of illogical persons that are obstinate. However, it has also been assumed that all obstinate persons are logical. How can the two be reconciled?

The obvious conclusion must be that there are no illogical persons. For if there were, they would have to be obstinate by the second premise. However, the prior assumption is that all the obstinate persons are philosophers and therefore logical. In other words, by said assumption, obstinate persons cannot be illogical. Therefore, the second premise can only apply if there are no illogical persons.

This conclusion may seem awkward. But it will be useful to demonstrate its veracity by means of a different example and afterwards return to the example just discussed.

The following two premises along with a conclusion are modeled after Ch. L. Dodgson's syllogism and concern all the students of a certain high school and an invitation to the students to participate in a $3 \mathrm{k}$ run to benefit a certain cause. In G. Boole's terms, the "universe of thought", or all that one could think about, is limited to these students. A more detailed analysis of the universe of thought will be presented later. The syllogism is as follows:

All runners will receive a certificate.

Those not receiving a certificate will be disappointed.

Therefore, some disappointed persons will not be runners.

If this type of syllogism is as mathematically inconclusive as it has been claimed to be above, then it ought to be possible that "None of the disappointed persons will not be runners". In other words, "The disappointed persons will all be runners". The notion of disappointed runners arises. However, by the first premise formulated above, all the runners will receive a certificate. Therefore, no runners can possibly be disappointed. How can this be?

The solution is obviously in this case that everyone ran. This possibility is easy to imagine. Why would not everyone want to run? That way everyone gets a certificate and no one is disappointed. The conclusion "None of the disappointed persons will not be runners" is therefore not contradicted because there are no disappointed persons.

It remains a fact that the statement "None of the disappointed persons will not be runners" is awkward. However, the statement was only postulated as a conclusion in an attempt to invalidate Ch. L. Dodgson's "some". In the end, no conclusion is possible from the two premises. Any postulated conclusion is therefore bound to be in some sense awkward.

One might also wonder what the point is of making a statement such as "The disappointed persons will all be runners" if it is possible for there to be no disappointed persons.

There have been two great tendencies in logic. Some have assumed that, if one makes a statement, the entities mentioned in the statement need to exist. Others have assumed that it does not matter whether they exist or not. I believe that the latter view is the correct one. However, it may be better to describe the latter view as follows: one does not focus at all on any possible existence on the part of any entities. What matters is the mathematical relation between the entities.

The hard core of logic is digital mathematics. In quantitative mathematics, the focus is often purely on a relation. Consider the Equation

$$
y=2 x,
$$

which is also a function. Both $x$ and $y$ could be any number. The Equation does not focus on any specific 
number. The focus is on the relation.

In a similar way, the focus of the Equation of digital mathematics is on a relation. What better way to demonstrate this focus than by means of an example in which an entity does not exist and yet a true statement is made about it. The statement is as follows:

Any human being who can lift two tons above his head is stronger than an elephant.

This statement is undeniably true. But there exist no such human beings. Another example of a true syllogism is as follows.

Premise 1: All horses with ten legs can fly.

Premise 2: Paris is a horse with ten legs.

Conclusion: Paris can fly.

Finally, it may be useful to return to the original example and consider what it means that "None of the obstinate persons are not philosophers", in other words, that "All the obstinate persons are philosophers". By the second of Ch. L. Dodgson's premises, all illogical persons are obstinate. If "All the obstinate persons are philosophers", then it follows that all illogical persons are philosophers. But by the first premise, philosophers are all illogical. How can this be?

The solution is that there are no illogical philosophers. Indeed, some might argue in favor of the view that human beings all have their philosophical moments and are in that regard all philosophers in a way. That also means by the first premise that they are all logical to an extent. In that sense, there are not really any illogical persons. One may chose to disagree with this view. But what matters is that it is logically possible to hold the view and think that one is making sense. Digital mathematics altogether allows for the possibility.

\subsection{Task Two: Identifying the Meaning of "Some Obstinate Persons Are Not Philosophers"}

The fact that the premises do not lead to the proposed conclusion in the first syllogism does not mean that there is nothing to be said about the conclusion or that no conclusions can be derived from the two premises. The conclusions are discussed in the next section. The present section is concerned with what the conclusion does mean.

The conclusion

Some obstinate persons are not philosophers. is best characterized by noting how it differs radically in nature from the conclusion

Socrates is mortal.

The difference is simply this. The former conclusion does not declare anything to be non-existent. It implies that there are obstinate philosophers, non-obstinate philosophers, obstinate non-philosophers, and non-obstinate non-philosophers. By contrast, the second conclusion declares the class of what is both Socrates and not mortal to be non-existent. The three classes containing what is Socrates and mortal, not Socrates but mortal, and neither Socrates nor mortal do otherwise exist.

To be appreciated in full, the difference between the two modes of declaration needs to be placed in the context of rational human intelligence in its entirety. The matter will be discussed in a follow-up paper. It will be eminently useful to introduce the concept of entropy to the study of rational human intelligence.

Entropy is a crucial component of the Second Law of Thermodynamics. It will appear that, in examining rational human intelligence, an essential structural principle can be adopted with great profit from the Second Law of Thermodynamics to the extent that it can be used to demonstrate how rational human intelligence is at all possible. Evidently, all this does not mean that the propagation of rational human intelligence has anything to do with the propagation of heat.

\subsection{Task Three: Conclusions That Do Derive from the Two Premises "All Philosophers Are Logical” and “An Illogical Man Is Always Obstinate”}

The conclusion that Ch. L. Dodgson derives from the two premises at hand is "Some obstinate persons are not philosophers". This conclusion conveys something that can presumably be known about obstinate people.

But it has been shown above in strictly mathematical terms that this specific knowledge cannot be derived from the premises in question.

In order to gain a better understanding of the premises in question, it will be useful to provide some more context by considering what does appear possible in terms of conclusions derived from the two premises.

In a rigorous mathematical system, it is necessary to determine the exact degree, not only of our knowledge, but also of our ignorance. Accordingly, a complete mathematical map of Ch. L. Dodgson's premises must be possible.

But before taking a look in $\S 14.11$ at the mathematical map in question, some remarks are in order about the values of two symbols of G. Boole's digital mathematics. The first symbol is "+". It corresponds to the Boolean operator OR. It is discussed in $\S 14.8$. The second symbol is $\frac{0}{0}$. It is discussed in $\S 14.9$ and $\S 14.10$.

In what follows, I will try to be fairly explicit about methodological principles for the sake of clarity. But it is difficult to see how a full understanding of the entire theoretical background is possible without revisiting the original works on digital mathematics by G. Boole and J. 
Venn.

The present elaborate analysis of Ch. L. Dodgson's syllogism may seem cumbersome or perhaps even overbearing. But I believe that full explicitness is necessary to impress upon the reader the total veracity, absolute finality, and consummate rigor of attributivity mathematics.

Some considerable claims have been made about the universality of attributivity mathematics, not in the least by G. Boole himself. Then again, so many lofty claims are being made all the time about all kinds of matters. The need is for positive indications of the validity of a certain method. Much of what follows in future papers regarding the structure of rational human intelligence will be built on the foundations of digital mathematics. It will therefore be useful to describe these foundations in more detail before proceeding to apply them to a comprehensive analysis of rational human intelligence.

\subsection{The Symbol “+” in G. Boole’s Algebra}

In Equation (5), namely

$$
p(1-l)+(1-l)(1-o)=0,
$$

the term to the left of the Equation mark has two components, namely "illogical philosophers" and $(1-l)(1-o)$ "illogical people that are not obstinate". Both components are said to not exist or be equal to zero $(=0)$.

It will be desirable to recast Equation (5) in terms of all three attributes $p, l$, and $o$. Accordingly, it can be rewritten as follows:

$$
\begin{aligned}
& {[p(1-l) o+p(1-l)(1-o)]} \\
& +[p(1-l)(1-o)+(1-p)(1-l)(1-o)] \\
& =0 .
\end{aligned}
$$

Equation (11) conveys above and beyond Equation (5) that illogical philosophers consist of both those that are obstinate $(o)$ and of those that are not obstinate $(1-o)$ and that illogical obstinate people consist of both those that are philosophers $(p)$ and those that are not philosophers $(1-p)$.

There are four terms in Equation (11). Each of the four components is formulated in terms of all three attributes. Together, the four terms denote all the non-existing $(=0)$ combinations of these three attributes in both their present and absent states. They will therefore be referred to in what follows as combination sets or classes.

Equation (11) may also be represented with all four terms on the same hierarchical level after elimination of the square brackets, as follows:

$$
\begin{aligned}
& p(1-l) o+p(1-l)(1-o) \\
& +p(1-l)(1-o)+(1-p)(1-l)(1-o) \\
& =0 .
\end{aligned}
$$

The most striking characteristic of Equation (12) is that the second component and the third component of the long term to the left of the Equation sign are one and the same, namely $p(1-l)(1-o)$.

It is at this juncture that, I believe, it is necessary to take a step that G. Boole did not himself always tend to take, for the simple reason that he did not always systematically formulate every component in terms of every attribute.

To some extent, the fact that he does not take this step affects the way in which he applies the comprehensive method that he himself for the first time so fully formulated. As a consequence, the application of his approach to examples does not always appear to me as smooth as it could be and certain problems even arise, as will be illustrated below.

No one considered the following fundamental axiom of digital mathematics to be as preeminent as G. Boole did, and rightly so:

$$
x \times x=x,
$$

which can also be written as

$$
x x=x \quad \text { [39], }
$$

which is the notation that $\mathrm{G}$. Boole prefers.

The real life purport of this axiom is as follows. If one takes, say, all the entities that exhibit the following two attributes, namely $f$ "French" and $f$ "French", what one obtains is still only what is French. In symbolic language,

$$
f \times f=f .
$$

This much for the symbol " $x$ ", the Boolean operator AND, and a fundamental axiom that pertains to it.

But similar reasoning is possible about the symbol "+", the Boolean operator OR. By the same appeal to real life purport, if one takes all the entities that are French and then, in a second act of selection, takes again all the entities that are French, what one obtains is still only what is French:

$$
f+f=f .
$$

However, I cannot readily locate anywhere in G. Boole's writings, nor in J. Venn's for that matter, any equivalent of the expression

$$
x+x=x .
$$

In fact, Th. Hailperin points to a letter by G. Boole to S. Jevons in which G. Boole categorically denies [40] that

$$
x+x=x .
$$

The correspondence between G. Boole and S. Jevons was first brought to light by Ph. E. B. Jourdain [41] as part of a series of useful articles on the history of mathematical logic $[42,43]$.

Instead, in the letter in question, G. Boole proposes 
that $[44,45]$

$$
x+x=0
$$

and furthermore that $x+x=0$ is equivalent to

$$
x=0 .
$$

What did G. Boole mean by $x+x=0$ ? He does not specify.

The purport of $x+x=0$ can be inferred, however, from how he understood the symbol "+". He interprets it as being exclusive. In other words, " $x+y$ " signifies that no $x$ is $y$ and no $y$ is $x$.

Therefore, in the expression $x+x$, the alternative to the first $x$ following + ought to be something that is not $x$. But it happens to be $x$. What is both $x$ and not $x$ at the same time must equal 0 by the fundamental law of thought.

And the same reasoning applies to the second $x$ in its relation to the first $x$. Another way of looking at this is as follows: If $x$ equals 0 in its second occurrence, then why would it not also in its first occurrence?

Equivalents of $x+x=x$ seem quite easy to find from the time onward when Boolean algebra came to be applied to the relay and switching circuits of electrical engineering, that is, from the late 1930s onward, in forms such as the following:

$$
1+1=1 .
$$

If there is a current in both of two parallel wires, there will evidently be a current in their joint output.

The symbol "+" denotes two electrical wires constructed next to one another in parallel. In electrical engineering, "1" can represent an On-current and " 0 " an Off-current.

But somewhat awkwardly, the opposite convention also exists. I have not quite appreciated this fact in my own earlier writings. According to this convention, " 0 " represents on On-current. " 0 " is understood as "zero resistance to an electric current" and hence as an On-current. Likewise, " 1 " is understood as "full resistance to an electric current" and hence as an Off-current. C. E. Shannon, who first applied G. Boole's algebra to electrical switching circuits in the late 1930s used this convention and possibly first introduced it.

In any event, I prefer the convention according to which " 1 " denotes an "On-current" and " 0 " and Offcurrent.

It seems more than obvious to me - as it has by now to most everyone involved - that G. Boole's exclusive interpretation cannot possibly stand. The elimination of this interpretation otherwise causes no detriment to $G$. Boole's general theory. J. Venn also advocated for the non-exclusive interpretation of "+". And in fact, a mere search on the Internet for $f+f$ "that which is French OR that which is French" yields that which is French and not 0

The exclusive interpretation of "+" seems to contradict the very internal coherence of G. Boole's theory. It results in untenable contradictions. For example, if one interprets the expression $x+y$ according to G. Boole's very own fundamental theorem of Development, mentioned above, one obtains the inclusive interpretation of the expression. The theorem is discussed below.

Then again, G. Boole perhaps unwittingly somehow comes close to $x+x=x$ when he discusses what is sometimes called the rule of absorption [46]. Thus, if $f$ is "French" and $d$ is "doctors", then

$$
f+f d=f .
$$

The real life purport of this Equation is evident. If one adds to what is French all the French doctors, one still only obtains what is French. In general, adding to something anything that belongs to that something still only produces that something.

The sum $x+x$ is only a special case of this general rule because the second $x$ belongs entirely to the first, and vice versa. By the mere rule of absorption, $x+x=x$. Elsewhere, G. Boole equates $1+w$ with 1 [47]. 1 is the symbol for everything. It is clear that everything smaller than everything itself belongs to 1 or everything. All that is not everything, which includes $w$, belongs to 1 and is absorbed by it, as it were.

It can also be noted that, in equating $x+x=0$ with $x=0, \mathrm{G}$. Boole himself in some sense equates $x+x$ with $x$. G. Boole recognizes that, in his exclusive interpretation of "+", $x+x=0$ can only be true if $x=0$.

If one accepts that $x+x=x$ and that $1+1=1$, then the Development of $x+x$ also yields $x$. The design of developing $x+x$ is to establish what part of $x$ and what part of non- $x$ is taken in $x+x$.

In sum, it seems imperative to reduce $p(1-l)(1-o)+$ $p(1-l)(1-o)$ in Equation (12) to just $p(1-l)(1-o)$. I can see no need to take the same class twice.

Then again, it is true that, if one promises $\$ 100$ to all the French and to all the doctors, the typical understanding will be that French doctors get \$200.

If "French" is $f$ and "doctors" are $d$, then G. Boole would represent the class encompassing all that is French and all the doctors as $f+d$ and assume that the two components of the class do not overlap.

But in the inclusive interpretation adopted here, the French encompass both the French non-doctors $(f \bar{d})$ and the French doctors $(f d)$ and the doctors encompass both the French doctors $(f d)$ and the non-French doctors $(\bar{f} d)$, and the French doctors are therefore in fact conceived of twice.

And indeed, if one develops the expression $f+d$ according to G. Boole's own formula of Development, one obtains inevitably $\bar{f} \bar{d}+f d+f d+\bar{f} d$, or what G. 
Boole and J. Venn would also write as $f \bar{d}+2 f d+\bar{f} d$. This expression appears to contradict the axiom just stated that $x+x=x$. Electrical engineers know that, in digital design, $1+1=1$ and not 2 . Why does the axiom somehow not seem to apply in the present case?

In this case, $x$ is "French doctors". Why are the French doctors counted twice and not reduced to just one count in accordance with the axiom $x+x=x$ or $f d+f d=f d$ ? The apparent contradiction is not addressed to full satisfaction or in full detail, I believe, in G. Boole's or J. Venn's writings. It will therefore be desirable to digress and propose an answer regarding the matter.

The reason for the apparent contradiction is the nature of the expression $f+d$. It is mathematically incomplete. The expression $f+d$ is something that G. Boole would altogether not hesitate to write. But there is something unsatisfying about it from the mathematical point of view. What is the origin of the expression?

The expression $f+d$ is a literal mathematical symbolic equivalent of the linguistic expression "French and doctors". There is no denying that "French and doctors" is a bona fide linguistic expression. Uttering the expression "the French and the doctors" is a bona fide linguistic act. The mind first conceives of the French, who encompass both French who are doctors and French who are not doctors, and it then conceives of the doctors, who encompass both the doctors who are French and the doctors who are not French.

The way in which the mind works through linguistic expression in real time inevitably involves the double contemplation of "French doctors". It is the separation in time between the utterance of the first instance and the utterance of the second instance that prevents the dual occurrence of "French doctors" to merge into just one, as digital mathematics would normally require on a strictly mathematical level. "French doctors" is clearly being contemplated twice.

There are cases in which there is no overlap between two terms of the expression $x+y$, as in the expression "squares and triangles". However, someone who does not know anything about squares or triangles would be entitled to assume that things that are both squares and traingles could also be signified by the expression. The knowledge that there are no such things is extraneous to the expression itself.

The symbolic expression $f+d$ in a sense mimics the linguistic expression "French and doctors". But in mathematical terms, the expression is an abbreviation of $\bar{f} \bar{d}+f d+f d+\bar{f} d$. Because of the abbreviation, both instances of $f d$ are prevented from merging into one, as it were.

In the end, I personally believe that, while the abbreviation is not entirely without its usefulness, it comes with a loss of mathematical precision. Full mathematical precision is always securely accomplished by spelling out all attributes in terms of all other attributes that are part of the expression in both their present and their absent states. Fully spelling out would in fact be necessary if the expressions in question were programmed for a computer.

A linguistic expression that represents French doctors only once is "all the French and all the non-French doctors". It is mimicked symbolically in abbreviation by $f+(1-f) d$. The complete mathematical representation is

$$
f d+f(1-d)+(1-f) d .
$$

Then there is the expression "all the French except the doctors and all the doctors except the French", which positively excludes French doctors. The complete mathematical representation is $f(1-d)+(1-f) d$.

I believe that there is every reason to reduce as $\mathrm{G}$. Boole would apparently not have (and in regard to which J. Venn nowhere expresses a definite opinion as far as I know) Equation (12) to

$$
p(1-l) o+p(1-l)(1-o)+(1-p)(1-l)(1-o)=0 .
$$

There are eight possible combinations of $p, l$, and $o$ in both their present and absent states. It appears from Equation (13) that three do not exist. It follows that the following five do exist: plo, $\bar{p} l o, p l \bar{l}, \bar{p} \bar{l}$, and $\bar{p} l \bar{o}$.

Incidentally, a notation such as $\bar{l}$ does offer the advantage of succinctness over $(1-l)$. Five symbolsnamely "(", "1", "-,", "l", and ")"- -are replaced with what is in effect just one symbol surmounted by a diacritic mark. However, for the purposes of the present investigation, it will very often be desirable to retain the longer, more transparent, notation. The transparency offered by the longer notation will serve the cause of clarity.

\subsection{G. Boole's Symbol for "Indefinite Amount"}

In Equation (5), no two symbols are more characteristic, some might say idiosyncratic, of G. Boole's algebra than $\frac{0}{0}$ and $\frac{1}{0}$. The symbol $\frac{0}{0}$ describes a class or set of which an indefinite amount is taken, that is, none, some, or all. The symbol $\frac{1}{0}$ describes a class or set that does not exist. The precise manner in which these symbols are generated by mathematical procedures will be described further below. The focus of the present section is on $\frac{0}{0}$.

It is no exaggeration to state that both symbols have mystified many, have been rejected by most, and have even been experienced as repulsive by some.

In his admirable biography of G. Boole, D. MacHale notes that G. Boole's "use of the 'indeterminate' symbol 
0/0 caused a great deal of difficulty" [48]. It sounds like a complete dismissal of the validity of the symbol. I have the impression that the author of the biography, being a mathematician, had a much better understanding of $\mathrm{G}$. Boole's work in quantitative mathematics than of his work in digital mathematics.

There is otherwise no doubt in my own mind of the total meaningfulness and absolute mathematical veracity of both $\frac{0}{0}$ and $\frac{1}{0}$.

Remarkably, G. Boole himself thought of them as being strictly mathematical and says little or nothing about their logical or "real world" significance. But J. Venn did much to show that they make perfect sense in terms of real life purport [49].

The mathematical significance of $\frac{0}{0}$ will be further elucidated below. Suffice it in this section to clarify the mathematical precision of "none, some, or all" by means of a simple example. If Paris is French, then what can be derived from this premise about French in terms of Paris?

It is clear that French includes all of Paris and none, some, or all of what is not Paris. J. Venn calls "none, some, or all of what is not Paris" a "surplus indefinite term" yielded by logical division, which is so characteristic of Boole's treatment. Probably no logician before Boole (with the very doubtful exception of H. Grassmann...) ever conceived a hint of this" [50]. And hardly anyone except J. Venn has put it to use since G. Boole. Yet, it is an essential component of digital mathematics.

The limit values "none" and "all" cannot be excluded if one knows absolutely nothing else about Paris or France.

For example, if the original Equation were instead that the square is a figure with four equal sides and four 90 degree angles, then figures with four equal sides and four 90 degree angles include all squares and none, some, or all what is not a square. In this case, the value "none" applies. There are no such figures outside squares.

If the original Equation were instead that, as regards the members of a soccer team, Jack is someone who likes a beer, then those who like beer on the team are Jack and none, some, or all of the others. In this case, the value "all" is altogether possible.

The first of the two premises of Ch. L. Dodgson's syllogism discussed above is that all philosophers are logical. On the basis of this statement, what can be said about logical persons? It can be said that they include all the philosophers and none, some, or all of the non-philosophers. "None" is the answer if one thinks of all human beings as in some way philosophical and therefore philosophers. "All" is the answer if one believes that there are really no logical persons and therefore also no phi- losophers. It is a view to which many will not want to subscribe. But the view can be held up and made sense of in a strictly mathematical sense.

The question "What is $\frac{0}{0}$ ?" can be formulated as follows:

$$
\frac{0}{0}=? .
$$

It follows from this Equation that

$$
? \times 0=0 \text {. }
$$

In other words, as J. Venn observed, what is being sought ("'?") is something that, when multiplied by 0 , yields 0 . What is sought could therefore be nothing, something, or everything, which all yield 0 when multiplied by 0 .

Likewise, it follows from

$$
\frac{1}{0}=?
$$

that

$$
? \times 0=1 .
$$

Evidently, that which yields 1 when multiplied by 0 does not exist.

It is not self-evident to think of none, some, or all as a concept of pure mathematics. It seems to go against the grain of mathematics, in which everything is supposed to be absolutely exact. And yet, as an item of knowledge, none, some, or all exhibits perfect mathematical exactitude.

\subsection{E. Schröder on G. Boole's "Indefinite Amount" and His Use of "Division" in Digital Mathematics}

E. Schröder, like just everyone else except J. Venn it seems, rejects the symbol $\frac{0}{0}$ and the use of the division symbol in digital mathematics in general.

He seems to fully understand the purport of $\frac{0}{0}$ and of the division symbol in general. Yet he rejects it for something else that seems related to it. But in doing so, he abandons mathematical precision while presumably somehow believing that he has gained it by getting rid of the vaguely defined "none, some, or all".

It should be noted right away that the division symbol does not have quite the same function as it has in quantitative mathematics. Everyone would therefore agree, it seems, E. Schröder foremost among them, that a name other than "division" would be suitable. Still, it remains a fact that, just as in quantitative mathematics, the division symbol signifies the inverse of what is commonly repre- 
sent in Boolean algebra as “×”, as J. Venn first and foremost made clear.

It should also be added that, obviously, what I and others call multiplication in digital mathematics is not the same as multiplication in quantitative mathematics. I use the term in reference to the Boolean operator AND.

In a biographical sketch of E. Schröder, J. Lüroth characterizes E. Schröder's abolition of the concept of division as a significant modification of G. Boole's system [51]. To sanction what he perceives as a departure from G. Boole's system, E. Schröder proposes an official designation for his own system. Writing in German, he even suggests to the reader what one should call it in English: "Boole's method as modified by me [that is, E. Schröder] (die von mir modifizirte Boole'sche Methode)" [52].

I will return in $\$ 21$ below to E. Schröder's approach when discussing examples as illustrations of the mathematical character of digital mathematics. Two preliminary observations are in order already here in the present section.

First of all, I cannot see in which way E. Schröder adds anything to G. Boole's system. I know of no problem that could be solved by E. Schröder's system and not by G. Boole's. It is true that the name "division" is not adequate. But the concept that it describes seems to me real, as will be illustrated with simple examples further below.

One might be tempted to consider the possibility that $\mathrm{E}$. Schröder thought of his system as just a more convenient formulation or conceptualization of what is essentially the same thing. But he is rather adamant about the importance of "eradicating (ausmerzen) division" from G. Boole's system [53]. One has the impression that he believes to have obtained a different mathematical outcome. Evidently, it is not possible to obtain two different results from the same operations. One must be wrong and the other right.

Second, I clearly see shortcomings in E. Schröder's approach. It will be useful to look at some simple statements further below in $\$ 21$ and ascertain that the result obtained for it according to G. Boole's and J. Venn's method is correct and also that the result obtained for it according to E. Schröder's method abandons a critical degree of absolute mathematical precision.

It is of some concern that someone like E. Schröder, who was an eminent mathematician and knew G. Boole's ideas so well and accepted them for the most part, could depart from them in some crucial respects. Such divergence can only undermine confidence in the whole enterprise of digital mathematics.

Everyone has a certain expectation of what mathematics is like. One is not supposed to be able to fudge matters in mathematics. Digital mathematics cannot be a matter of opinion or individual choice if it is to be truly mathematics. Digital mathematics is not as widely practiced as simple arithmetic. In simple arithmetic, everyone can readily agree that 2 plus 2 is not 5 . But it takes a little more careful attention to ascertain that the same applies, mutatis mutandis, to digital mathematics. The design of examples adduced further below in $\$ 21$ is to demonstrate that E. Schröder did something like declaring 5 to be the sum of 2 plus 2 .

Some general characteristics of E. Schröder's approach are in order already in this section, lest my rejection of his approach to what is represented by G. Boole symbolically as division give a wrong impression of my appreciation of E. Schröder's work as a whole.

First, E. Schröder was by all accounts a highly competent mathematician who at some point served as director of the Technische Hochschule in Karlsruhe.

Second, with the sole exception of J. Venn, no one else beside him understood, absorbed, and assimilated G. Boole's ideas as completely as far as I know. Simply put: J. Venn and E. Schröder fully understood G. Boole, and relatively few others ever did.

Third, E. Schröder's disquisitions are as a rule accurate and very exhaustive even if, on a more subjective note, I have the impression that his lines of argument can on occasion be so belabored that a certain degree of elegance is lost.

I should add that this observation is limited to the first of the three books of his "Vorlesungen über die Algebra der Logik". I am less acquainted with the second and third books [54]. In the latter two books, E. Schröder moves on to something entirely different, something beyond what is addressed by G. Boole's system. What E. Schröder writes in them is strongly influenced by the writings of Ch. S. Peirce. Many, if not all, find Ch. S. Pierce's writings to be often obscure, and E. Schröder is no exception [55].

There is a sharp contrast between book one, on the one hand, and books two and three, on the other hand. It is generally possible to give real life interpretations of the digital mathematics of book one, just as one can of G. Boole's system, often with not a little help from J. Venn. By contrast, books two and three seem much more abstract.

At the beginning of book two, E. Schröder describes the works that have inspired him to write the book as presenting on occasion "hieroglyphic" systems ("Hieroglyphen"systeme) [56]. And it is not meant as a complement. Books two and three will in fact play no role in the present effort to describe rational human intelligence.

The ideas of books two and three remind one of the intellectual movement that lasted from 1870 to 1940, and beyond, to find the roots of mathematics. I. GrattanGuinness has described the evolution of this movement 
in the greatest detail [57]. The many valiant attempts that he records deserve commendation. And so are his own efforts to get deep inside the many tentacles of the movement. But in the end, to me, the description of much of this movement reads like a record of failure. After all, after a 690-page book entitled "The Search for Mathematical Roots", one might feel tempted to find at the end an answer to the simple question: So what are the roots of mathematics? However, there is no declaration to the effect of "In sum, the roots of mathematics are: ..."

Fourth, one cannot help to note that E. Schröder's work is somewhat underappreciated, even if this observation cannot be extended to I. Grattan-Guiness's aforementioned book, which treats his work in detail. I. Grattan-Guinness was also struck by the small impact of E. Schröder's work [58]. From the papers of C. LaddFranklin kept at Columbia University, I. Grattan-Guinness retrieved the following quote about E. Schröder's three-volume "Vorlesungen" by J. N. Keyes, the father of the much better known J. M. Keynes, the economist, who was himself no mean student of logic and who predeceased his father. According to the quote, the work "is rather full of stupidities, but the core is sound", which I. Grattan-Guinness characterizes as "typical Cambridge snobbery".

What explains this neglect? Two facts may have contributed to it.

First, the three tomes of E. Schröder's "Vorlesungen über die Algebra der Logik" are dense and weighty, at 721, 606, and 649 pages respectively, for a total of 1976 pages. Second, the percentage of academics competent in German has declined over the decades.

One facet of E. Schröder's work that has had little or no impact is his opposition to G. Frege's much celebrated Begriffsschrift [59]. G. Frege has now become something of a cult hero in certain quarters. The degree of enthusiasm for G. Frege is such that anyone who wishes to voice opposition may want to think twice. Yet, after some reflection, I personally cannot share the enthusiasm. I provisionally believe that it will be possible to program a computer to mimic rational human intelligence based on G. Boole's ideas and this and future papers are designed to describe some of the basic tools. In fact, all that computers can do so far is based on those ideas. I cannot see any need for G. Frege's ideas in such an endeavor.

\subsection{The Mathematical Map of the Two Premises in Ch. L. Dodgson's Syllogism}

The two premises of Ch. L. Dodgson's afore-mentioned syllogism do not yield the conclusion that he thinks that they do. Then again, there are conclusions that are possible. In fact, since those conclusions are obtained by purely mathematical methods, a complete mathematical map of them should be possible. This map should detail with absolute rigor the exact measure of what is known and what is not known.

Suffice it here to take note of some characteristics of the map by means of a couple of examples.

Ch. L. Dodgson's false conclusion defines obstinate persons $(o)$. It is therefore natural to ponder what kind of conclusions can be inferred mathematically from the two premises about $o$, and also about the complement of $o$, namely non- $O$ or $1-o$ or $\bar{o}$.

Considering that

$$
p(1-l) \times(1-o)
$$

is the same as

$$
[p(1-l) \times 1]-[p(1-l) \times o],
$$

or also

$$
p(1-l)-p(1-l) o
$$

and that

$$
(1-p)(1-l) \times(1-o)
$$

is the same as

$$
[(1-p)(1-l) \times 1]-[(1-p)(1-l) \times o],
$$

or also

$$
(1-p)(1-l)-(1-p)(1-l) o
$$

Equation (13) above can be rewritten as follows:

$$
\begin{aligned}
& p(1-l) o+p(1-l)-p(1-l) o \\
& +(1-p)(1-l)-(1-p)(1-l) o \\
& =0 .
\end{aligned}
$$

From (14), it follows that

$$
\begin{aligned}
& p(1-l) o-p(1-l) o-(1-p)(1-l) o \\
& =-p(1-l)-(1-p)(1-l)
\end{aligned}
$$

And since

$$
p(1-l) o-p(1-l) o=0,
$$

Equation (15) can be rewritten as

$$
-(1-p)(1-l) o=-p(1-l)-(1-p)(1-l),
$$

and hence as

$$
o=\frac{-p(1-l)-(1-p)(1-l)}{-(1-p)(1-l)},
$$

and therefore also as

$$
o=\frac{p(1-l)+(1-p)(1-l)}{(1-p)(1-l)} .
$$

The next step is to "develop" the right-hand term in 
(16).

As has already been noted above, Development is a term introduced by G. Boole to denote one of what are, as he describes it, the three main operations of digital mathematics: Development, Elimination, and Reduction.

The aim of Development is to convert a digital mathematical expression in terms of the sum of all the possible products of the attributes in their present and absent states. In Equation (16), the attributes are $p$ and $l$. The sum of the products of these two attributes in their present and absent states is therefore as follows:

$$
p l+p(1-l)+(1-p) l+(1-p)(1-l) .
$$

If one develops the right-hand size of Equation (16), one obtains as a result what $o$ is in relation to the four classes "logical philosophers", "non-logical philosophers", "logical non-philosophers", "non-logical nonphilosophers". In other words, what needs to be taken from these four sets or classes in order to obtain $o$ and exactly $o$ ? Or, what part exactly of these four sets or classes is $o$ ?

G. Boole obtains this result by means of his universal formula of development, which is a theorem of which he presents a mathematical proof [60].

In its most general formulation, the formula is as follows:

$$
f(x)=f(1) x+f(0)(1-x) .
$$

By means of this formula, any digital mathematical expression containing the attribute $x$ can be recast into an equivalent that states what part of $x$ and what part of non- $x$ needs to be taken in order to obtain the expression. The result may also be called an expansion of the expression containing the attribute $x$.

When a digital mathematical expression containing two attributes $x$ and $y$ needs to be expanded in terms of both attributes, the formula is rather as follows:

$$
\begin{aligned}
f(x, y)= & f(1,1) x y+f(1,0) x(1-y) \\
& +f(0,1)(1-x) y \\
& +f(0,0)(1-x)(1-y)
\end{aligned}
$$

The right-hand term in Equation (16) involves two attributes, namely $p$ and $l$. Accordingly,

$$
f(p, l)=\frac{p(1-l)+(1-p)(1-l)}{(1-p)(1-l)} .
$$

The development of the right-hand term can be rewritten as follows:

$$
\begin{aligned}
f(p, l)= & f(1,1) p l+f(1,0) p(1-l) \\
& +f(0,1)(1-p) l \\
& +f(0,0)(1-p)(1-l) .
\end{aligned}
$$

In the expressions $f(1,1), f(1,0), f(0,1)$, and $f(0,0)$, the first digit of the two digits in each term pertains to $p$ and the second digit pertains to $l$. The digits signify the value that $p$ and $l$ assume in the development or expansion of the right-hand term in Equation (16). Accordingly,

$$
\begin{aligned}
f(1,1) & =\frac{1(1-1)+(1-1)(1-1)}{(1-1)(1-1)} \\
& =\frac{1 \times 0+0 \times 0}{0 \times 0}=\frac{0+0}{0}=\frac{0}{0} .
\end{aligned}
$$

By this same procedure, it is possible to reformulate Equation (17) as follows, substituting $p$ and $l$ by 1 or 0 in accordance with the formula of Development:

$$
\begin{aligned}
o= & f(p, l)=\frac{p(1-l)+(1-p)(1-l)}{(1-p)(1-l)} \\
= & \frac{1(1-1)+(1-1)(1-1)}{(1-1)(1-1)} \times p l \\
& +\frac{0(1-1)+(1-0)(1-1)}{(1-0)(1-1)} \times(1-p) l \\
& +\frac{0(1-0)+(1-0)(1-0)}{(1-0)(1-0)} \times(1-p)(1-l) .
\end{aligned}
$$

The four components of the right-hand term of Equation (18) can be simplified as follows:

$$
\begin{aligned}
o= & \frac{1 \times 0+0 \times 0}{0 \times 0} \times p l+\frac{1 \times 1+0 \times 1}{0 \times 1} \times p(1-l) \\
& +\frac{0 \times 0+1 \times 0}{1 \times 0} \times(1-p) l+\frac{0 \times 1+1 \times 1}{1 \times 1} \times(1-p)(1-l) .
\end{aligned}
$$

And hence further as follows:

$$
\begin{aligned}
o= & \frac{0}{0} \times p l+\frac{1}{0} \times p(1-l)+\frac{1}{0} \times p(1-l) \\
& +\frac{1}{1} \times(1-p)(1-l) .
\end{aligned}
$$

And finally, with additional simplification and also a reordering of the components, as follows:

$$
\begin{gathered}
o=(1-p)(1-l)+\frac{0}{0} p l+\frac{0}{0}(1-p) l ; \\
\text { with } p(1-l)=0 .
\end{gathered}
$$

Or, with abbreviated notation, also as follows:

$$
o=\bar{p} \bar{l}+\frac{0}{0} p l+\frac{0}{0} \bar{p} l ; \text { with } p \bar{l}=0 .
$$

According to (19), obstinate persons consist of the following three sets or classes: all illogical non-philosophers; none, some, or all of the logical philosophers; and none, some, or all of the logical non-philosophers. 
In other words, obstinate persons include all the illogical non-philosophers and for the rest are part of the logical persons, whether as philosophers or not.

Further shortening is possible. As regards the first of the three components of the right-hand term in (19), it follows from the first premise of Ch. L. Dodgson's syllogism that

$$
p \bar{l}=0 \text { "Illogical philosophers do not exist". }
$$

Therefore,

$$
\bar{p} \bar{l}=\bar{l} \text {. }
$$

The purport of Equation (20) is that "Illogical nonphilosophers are the same as the totality of non-philosophers", which is the same as stating that all illogical persons are non-philosophers (but not the other way around).

As regards the second and third components of Equation (19), it can be said that

$$
\bar{p} \bar{l}+p \bar{l}=\bar{l} .
$$

Putting (21) into words, the illogical philosophers and the illogical non-philosophers together naturally make up all the illogical persons.

Accordingly, Equation (19) can be shortened to

$$
o=\bar{l}+\frac{0}{0} l .
$$

According to (22), obstinate persons include all the illogical persons and for the rest are part of the logical persons, that is, are the same as none, some, or all of the logical persons.

Equation (22) defines the relation between $o$ and just $l$ in both its present and absent states and not, as in (19), the relation between $o$ and both $p$ and $l$ in their present and absent states.

Equation (19) can also be simplified so that it is determined what $o$ is in relation to only $p$ in both its present and absent states.

If one considers, (1), that

$$
\bar{p} \bar{l}+\frac{0}{0} \bar{p} l=\frac{0}{0} \bar{p},
$$

that is, that all the illogical non-philosophers and none, some, or all of the logical non-philosophers are the same as none, some, or all of the non-philosophers, and (2), that

$$
\frac{0}{0} p l=\frac{0}{0} p,
$$

that is, that none, some, or all of the logical philosophers are at the same time none, some, or all of the philosophers, then Equation (19) can be rewritten as

$$
o=\frac{0}{0} p+\frac{0}{0} \bar{p} \text {. }
$$

This means that obstinate persons are none, some, or all of the philosophers and none, some, or all of the non-philosophers. This is completely undefined. Obstinate persons could be all of the former or all of the latter or also none of either the former or the latter.

Equation (23) confirms the result obtained above, namely that the relation between $o$ and $p$ is completely undefined according to Ch. L. Dodgson's two premises.

How does Equation (19) differ from Ch. L. Dodgson's result, according to which some obstinate persons are not philosophers?

It appears that two of the three classes in Equation (19) that make up the obstinate persons contain non-philosophers. One might conclude that Ch. L. Dodgson's conclusion that some obstinate persons are non-philosophers is confirmed. It is not.

The matter has been discussed at length above. But the gist of this discussion may be briefly recalled here. It cannot be excluded that everyone is considered philosophical in some sense and therefore qualifies in a way as a philosopher and hence also as a logical person. One may disagree with this view. But it cannot be denied that the view can be held. The obstinate persons would then all be logical philosophers $(p l)$ and none would be nonphilosophers, contrary to Ch. L. Dodgson's conclusion.

Then again, someone may be inclined to assume that there are no philosophers, either in general or in the group of persons to which Equation (19) is applied. In that case, all the obstinate persons would be non-philosophers, also contradicting Ch. L. Dodgson's conclusion.

So far, it has been established what $o$ is in terms of both $p$ and $l$ or either just $p$ or just $l$ in their present and absent states.

By the same procedure, it is possible to establish what non- $o$ is in relation to $p$ and $l$, what $p$ and non- $p$ are in terms of $l$ and $o$, and what $l$ and non- $l$ are in terms of $p$ and $o$.

The mathematical derivations cannot be laid out all in detail here according to the rules described above. The results will need to suffice for the most part. But it will be useful to detail the steps for $\bar{o}$. The result for $\bar{o}$ is as follows:

$$
\begin{gathered}
\bar{o}=\frac{0}{0} p l+\frac{0}{0} \bar{p} l ; \text { with } p \bar{l}=0 ; \\
\text { and taking none of } \bar{p} \bar{l} .
\end{gathered}
$$

"Non-obstinate persons consist of none, some, or all of the logical persons, whether they are philosophers or not. There are no illogical philosophers. And none of the illogical non-philosophers are included if there are any".

The component "persons" in "obstinate persons" is inferred because obstinate is not typically associated with things. It is assumed that it is clear from the context that 
one is talking of people only.

The above result contains an example of the coefficient 0 , which accompanies the class of illogical nonphilosophers. The coefficient 0 does not signify that the class that it accompanies does not exist. It may or it may not. But if it does, 0 signifies that none of it is included among the non-obstinate.

Another way of putting (24) into words is as follows:

Non-obstinate persons are all logical, whether they are philosophers or not.

Equation (24) can be reformulated in terms of just $l$ as follows:

$$
\bar{o}=\frac{0}{0} l .
$$

"Non-obstinate persons are all logical".

Equation (24) can be reformulated in terms of just $p$ as follows:

$$
\bar{o}=\frac{0}{0} p+\frac{0}{0} \bar{p} .
$$

"Non-obstinate persons are or are not philosophers".

Equation (25) is entirely inconclusive. Indeed, it can be reformulated as follows:

$$
\bar{o}=\frac{0}{0}(p+\bar{p}) .
$$

And since

$$
p+\bar{p}=1 \text {. }
$$

"Everything (1) is either a philosopher or not a philosopher",

Equation (26) is the same as

$$
\bar{o}=\frac{0}{0} \text {. }
$$

This means that $\bar{o}$ is completely undefined. Equation (27) can be reformulated as

$$
\bar{o} \times 0=0 .
$$

It appears that, if nothing is taken of $\bar{o}(\times 0)$, the result is nothing (0). In other words, $\bar{o}$ could be anything.

The steps by which (24) is derived from Equation (13) above are as follows. Note especially the expression $1-\bar{o}$ below.

From

$$
\begin{aligned}
& p(1-l) o+p(1-l)(1-o) \\
& +(1-p)(1-l)(1-o)=0
\end{aligned}
$$

it follows that

$$
\begin{aligned}
& p(1-l)(1-\bar{o})+p(1-l)(1-o) \\
& +(1-p)(1-l)(1-o)=0 .
\end{aligned}
$$

Note that $o$ has been replaced by $1-\bar{o}$, which is the same as $1-(1-o)$, or also as $1-1+o$, and therefore as just $o$.
Since

is the same as

$$
p(1-l)(1-\bar{o})
$$

$$
p(1-l)-p(1-l) \bar{o}
$$

and therefore also as

$$
p(1-l)-p(1-l)(1-0),
$$

Equation (28) can be rewritten as

$$
\begin{aligned}
& p(1-l)-p(1-l)(1-o)+p(1-l)(1-o) \\
& +(1-p)(1-l)(1-o)=0,
\end{aligned}
$$

which simplifies to

$$
p(1-l)+(1-p)(1-l)(1-o)=0 .
$$

Bringing all terms except $1-o$ over to the other side of the Equation gives

$$
1-o=\frac{-p(1-l)}{(1-p)(1-l)} .
$$

This expression seems puzzling at first sight. It looks like a class is being subtracted from something that is not there. It needs to be remembered, however, that digital mathematics is concerned. Equation (30) derives from Equation (29), in which a sum of two terms is declared equal to 0 . In digital mathematics, this means that either term is also 0 . Adding 0 is the same as subtracting 0 . Nothing changes. In that regard, $p(1-l)$ and $-p(1-l)$ relate to one another as +0 does to -0 . Equation (30) is therefore equivalent to

$$
1-o=\frac{p(1-l)}{(1-p)(1-l)} \text {. }
$$

Another approach is more purely mathematical, namely squaring the right-hand side of Equation (30). In digital mathematics, $x=x x$. Accordingly, $(-x)(-x)$ would be equal to $+x$.

G. Boole seems to have had no difficulty with turning a product of two negated terms into a positive term as is done in quantitative mathematics, in the sense that $(-1)(-1)=+1$ [61]. The procedure appears to be successful. But a more detailed investigation of this concept seems desirable.

For the time being, certain tests of the system's coherence seem feasible. According to (31), $1-o$ would need to be something that yields $p(1-l)$ if it is multiplied by $(1-p)(1-l)$. From Equation (31), it follows that

$$
(1-o)(1-p)(1-l)=p(1-l) .
$$

According to Equation (24),

$$
\bar{o}=\frac{0}{0} p l+\frac{0}{0} \bar{p} l .
$$


Inserting Equation (24) into Equation (32) yields the following result:

$$
\left(\frac{0}{0} p l+\frac{0}{0} \bar{p} l\right) \times(1-p)(1-l)=p(1-l) .
$$

Equation (33) features to the left of the Equation sign the multiplication of $l$ "logical" with $(1-l)$ "non-logical". According to the fundamental axiom of thought, which holds that something cannot be something and not be something at the same time, something that is both something and not something equals 0 . In mathematical notation,

$$
l(1-l)=0 .
$$

It follows that the term to the left of the Equation sign in (33) equals 0 , or

$$
0=p(1-l) .
$$

Equation (34) yields no contradiction, as Equation (5) has already revealed that $p(1-l)$ equals 0 .

Developing the right-hand term of

$$
1-o=f(p, l)=\frac{p(1-l)}{(1-p)(1-l)}
$$

yields

$$
\begin{aligned}
& \frac{1(1-1)}{(1-1)(1-1)} \times p l+\frac{1(1-0)}{(1-1)(1-0)} \times p(1-l) \\
& +\frac{0(1-1)}{(1-0)(1-1)} \times(1-p) l+\frac{0(1-0)}{(1-0)(1-0)} \times(1-p)(1-l),
\end{aligned}
$$

and therefore also

$$
\begin{aligned}
& \frac{1 \times 0}{0 \times 0} p l+\frac{1 \times 1}{0 \times 1} p(1-l)+\frac{0 \times 0}{1 \times 0}(1-p) l \\
& +\frac{0 \times 1}{1 \times 1}(1-p)(1-l) .
\end{aligned}
$$

The final result, already mentioned as Equation (24) above, is as follows:

$$
\bar{o}=\frac{0}{0} p l+\frac{0}{0} \bar{p} l ; p \bar{l}=0 ; \text { taking none of } \bar{p} \bar{l} .
$$

This still leaves $p$ "philosophers", $\bar{p}$ "non-philosophers", $l$ "logical persons", and $\bar{l}$ "illogical persons" to be defined. The definitions in question, along with a few comments about some peculiar characteristics, are as follows.

As regards $p$ :

$$
p=\frac{0}{0} l o+\frac{0}{0} l \bar{l} ; \bar{l} \bar{o}=0 ; \text { taking none of } \bar{l} o .
$$

"Philosophers consist of none, some, or all of the logical persons whether they are obstinate or not. There are no non-obstinate illogical persons. None of the illogical persons are included".
The main Equation in (35) can be rephrased as follows:

Philosophers are logical, whether they are obstinate or not.

At first, it may seem as if Equation (35) does not yield much more information than the first of Ch. L. Dodgson's two premises, namely "Philosophers are logical". Still, it is interesting to know that philosophers can be either obstinate or not obstinate. After all, there is the possibility that either of these two options might be excluded. All depends on what the second premise holds, in which the attribute "obstinate" is mentioned.

The attribute "philosopher" is linked to the attribute "logical" in the first premise. The attribute "logical" is linked in its absent state "illogical" to the attribute "obstinate" in the second premise. Accordingly, there is the possibility that, through the attribute "(il)logical", the attribute "obstinate" may somehow impose some kind of a restriction on the attribute "philosopher".

However, the mention of "obstinate" in the second premise does not lead to the conclusion that philosophers cannot be obstinate or that they cannot be non-obstinate. They could be either.

The second component of Equation (35) indicates that there are no illogical persons that are not obstinate. That follows directly from the second premise. The second premise does otherwise not exclude the possibility that there are illogical persons that are obstinate. It states that illogical persons are all obstinate. Therefore, if there are any illogical persons, they are definitely obstinate.

As regards $\bar{p}$ :

$$
\bar{p}=\overline{l o}+\frac{0}{0} l o+\frac{0}{0} l \bar{o} ; \bar{l} \bar{o}=0 .
$$

"Non-philosophers consist of all illogical obstinate persons and none, some, or all of the logical persons whether they are obstinate or not. There are no illogical persons that are not obstinate".

It appears that the definition of non-philosophers is rather wide-ranging.

In relation to the two attributes "logical" or "obstinate", they can be obstinate only or logical only or both obstinate and logical at the same time.

But they cannot be illogical and not obstinate at the same time. The simple reason is that, by the second premise, illogical persons that are not obstinate do not exist.

Non-philosophers include all those that are illogical and obstinate because it follows from the first premise that all philosophers are logical that all illogical persons are non-philosophers.

As regards $l$ and $\bar{l}$ :

$$
l=p o+p \bar{o}+\bar{p} \bar{o}+\frac{0}{0} \bar{p} o
$$

"Everyone is logical except none, some, or all of the 
obstinate non-philosophers";

$\bar{l}=\frac{0}{0} \bar{p} o ;$ none taken from $p o, p \bar{o}, \bar{p} \bar{o}$, which all exist

"Illogical people are all obstinate non-philosophers".

The result for $l$ is interesting because all four possible classes are included, three of them completely. By contrast, $\bar{l}$ consists of only one class and then possibly only some or even none of it.

Naturally, $l$ and $\bar{l}$ ought to add up to everything (that is, " 1 " in G. Boole's algebra). One is either logical or one is not. In mathematical notation:

$$
l+\bar{l}=1 .
$$

Indeed, if one adds up the results for $l$ and $\bar{l}$ detailed above, the sum is all four possible combinations of the attributes "philosopher" and "obstinate" in their present and absent states: three combinations belong entirely to the result for $l$ and one combination, $\bar{p} o$ "obstinate non-philosophers", belongs either entirely to $l$ or $\bar{l}$ or in an unknown distribution to both at the same time.

There is much more detail that can be specified in relation to the mathematical map of Ch. L. Dodgson's two premises. One facet of this map is as follows. Each of the three attributes $p, l$, and $o$ in both their present and absent states has been defined above in relation to the two other attributes. The result is six definitions. The question arises: Is it possible to derive from any of these six definitions the five other definitions? It appears that it is.

Naturally, there are $30(6 \times 5)$ different cases to be distinguished. However, only one case will be considered for the sake of brevity. May it suffice to illustrate how the definition of logical persons $(l)$ can be derived from the definition of the non-philosophers $(\bar{p})$.

Deriving one definition from another poses special problems. Considered by themselves, the Equations that are part of the definitions exhibit a certain loss of information when compared to the original premises. This loss of information shows up when one tries to derive one definition from one another. The present example is meant to illustrate this.

From the first Equation in (36), namely

$$
\bar{p}=\bar{l} o+\frac{0}{0} l o+\frac{0}{0} l \bar{o},
$$

the following Equations follow in sequence in derivational fashion:

$$
\begin{gathered}
\bar{p}=\overline{l o}+\bar{p} l o+\bar{p} l \bar{o} ; \\
\bar{p}=(1-l) o+\bar{p} l o+\bar{p} l \bar{o} ; \\
\bar{p}=o-l o+\bar{p} l o+\bar{p} l \bar{o} ; \\
\bar{p}-o=-l o+\bar{p} l o+\bar{p} l \bar{o} ; \\
\bar{p}-o=\bar{p} l o+\bar{p} l \bar{o}-l o ;
\end{gathered}
$$

$$
\begin{gathered}
\bar{p}-o=l(\bar{p} o+\bar{p} \bar{o}-o) \\
l=\frac{(1-p)-o}{(1-p) o+(1-p)(1-o)-o} .
\end{gathered}
$$

Developing the right-hand side produces

$$
\begin{aligned}
l= & \frac{(1-1)-1}{(1-1) 1+(1-1)(1-1)-1} \times p o \\
& +\frac{(1-1)-0}{(1-1) 0+(1-1)(1-0)-0} \times p \bar{o} \\
& +\frac{(1-0)-1}{(1-0) 1+(1-0)(1-1)-1} \times \bar{p} o \\
& +\frac{(1-0)-0}{(1-0) 0+(1-0)(1-0)-0} \times \bar{p} \bar{o} .
\end{aligned}
$$

And hence

$$
l=1 \times p o+\frac{0}{0} \times p \bar{o}+\frac{0}{0} \times \bar{p} o+1 \times \bar{p} \bar{o} .
$$

Or also

$$
l=p o+\frac{0}{0} p \bar{o}+\bar{p} \bar{o}+\frac{0}{0} \bar{p} o .
$$

But the result obtained above for $l$ is

$$
l=p o+p \bar{o}+\bar{p} \bar{o}+\frac{0}{0} \bar{p} o .
$$

Why is the result obtained here less precise, yielding an indefinite amount of $p \bar{o}$ (which could be none, some, or all of it) and not exactly all of it?

The reason is that the definition of $l$ has been derived from the first of the two Equations in (36). In the derivation of Equation (36) from two premises, the information obtained is distributed over two Equations. Therefore, when the definition of $l$ is derived from the first Equation only in (36), the result is less precise because it has been derived from incomplete informations.

Additional information is provided in Equation (36) through the second of the two Equations, namely

$$
\bar{l} \bar{o}=0 \text {. }
$$

This additional information makes it possible to sharpen

$$
l=p o+\frac{0}{0} p \bar{o}+\bar{p} \bar{o}+\frac{0}{0} \bar{p} o
$$

into

$$
l=p o+p \bar{o}+\bar{p} \bar{o}+\frac{0}{0} \bar{p} o,
$$

as follows.

The Equation

$$
\overline{l o}=0
$$

can be rewritten as 


$$
(1-l) \bar{o}=0,
$$

and hence as

$$
\bar{o}-l \bar{o}=0
$$

and as

$$
\bar{o}=l \bar{o}
$$

or

$$
\bar{o}=\bar{o} l .
$$

According to this last Equation, all non-obstinate people are logical. This must include both the non-obstinate persons that are philosophers and those that are not philosophers. Non-obstinate philosophers, or $p \bar{o}$, are therefore all logical.

However,

$$
l=p o+\frac{0}{0} p \bar{o}+\bar{p} \bar{o}+\frac{0}{0} \bar{p} o
$$

states that an indefinite amount of them is logical. The "indefinite amount" needs to be changed into "all". The result is

$$
l=p o+p \bar{o}+\bar{p} \bar{o}+\frac{0}{0} \bar{p} o .
$$

It would be desirable to study the loss of information at hand. But that needs to be reserved for a future paper.

One more simple example involving loss of information but only a single original Equation and not two may suffice at present.

Consider the Equation

$$
f=d b
$$

"The Flemish are the Dutch-speaking Belgians".

If one first derives from this Equation what Belgians are in terms of the Flemings and the Dutch speakers and then derives from the resulting definition of the Belgians what the Flemings are, the final resulting definition of the Flemings will be less precise than the original definition that served as point of departure.

The definition of the Belgians is as follows:

$$
b=f d+\frac{0}{0} \bar{f} \bar{d} ; \text { with } f \bar{d}=0 .
$$

"The Belgians include all the Flemish, who are Dutch speakers, as well as none, some or all of those that are neither Flemish nor Dutch speakers. Furthermore, there are no Flemings that do not speak Dutch".

The definition of the Flemings derived from the definition of the Belgians is as follows:

$$
f=d b+\frac{0}{0} \bar{d} \bar{b}
$$

"The Flemish include all the Dutch-speaking Belgians, as well as none, some, or all of those that are neither
Dutch-speaking nor Belgians".

The loss of information can be precisely defined. One expects no less than this kind of precision from mathematical operations.

In the original definition of the Flemings, four combination classes are equal to 0 . In the final definition, only three are. The loss concerns the combination class $f \bar{d} \bar{b}$ "Flemings that are neither Dutch-speaking nor Belgians". The fact that this class is equal to 0 is essential to the original definition of the Flemish. But it is no longer relevant when a definition of the Belgians is derived from the original definition of the Flemish.

Once this information is lost, it cannot be retrieved when a definition of the Flemings needs to be derived from the definition of the Belgians.

The information can be retrieved from the second Equation that results, namely

$$
f \bar{d}=0 .
$$

"There are no Flemings that do not speak Dutch". When this information is added to the definition

$$
f=d b+\frac{0}{0} \bar{d} \bar{b}
$$

"The Flemish include all the Dutch-speaking Belgians, as well as none, some, or all of those that are neither Dutch-speaking nor Belgians",

the second term of the expression to the right of the Equation mark is affected. The set or class in question cannot contain Flemings because it consists of non-Dutch speakers.

This much for the phenomenon of loss of information. Three additional remarks concerning certain peculiarities of the mathematical map of Ch. L. Dodgson's syllogism are in order.

First, the numerator of an expression may be 0 , as in the following expression, which plays a role in obtaining the definition of $\bar{l}$ :

$$
\frac{0}{p o+p(1-o)+(1-p)(1-o)} \text {. }
$$

In quantitative mathematics, this entire expression equals 0 , but not in non-quantitative, digital mathematics. Developing this expression yields the definition for $\bar{l}$ provided above.

Second, Venn diagrams are generally helpful in grasping the real life purport of all the definitions formulated above.

Then again, Venn diagrams involve a loss of information. For example, the two propositions "Philosophers are all logical" and "Illogical people are all non-philosophers" are both represented by the same Venn diagram. The diagram consists of two overlapping circles, one representing the philosophers and the other the logical persons. The section of the circle representing the phi- 
losophers that does not overlap with the circle representing to logical persons is empty.

The two propositions are therefore what may be called logically equivalent. There is no way in which Venn diagrams can represent the two statements differently.

Third, according to Equation (22),

$$
o=\bar{l}+\frac{0}{0} l .
$$

From Equation (36), namely

$$
\bar{p}=\bar{l} o+\frac{0}{0} l o+\frac{0}{0} l \bar{o},
$$

it follows that

$$
\bar{p}=\bar{l}+\frac{0}{0} l .
$$

Equations (22) and (37) at first sight may make it seem as if $o$ and $\bar{p}$ are one and the same or fully overlap because they are defined in the same way. However, they only partially overlap.

There are eight combination sets or classes involving the three attributes $p, l$, and $o$ in their present and absent states. Three are empty, to wit $p \overline{l o}, p \bar{l} \bar{o}$, and $\bar{p} \bar{l} \bar{o}$, and five are occupied, to wit plo, $\bar{p} l o, p l \bar{c}, \bar{p} \overline{l o}$, and $\bar{p} l \bar{o}$. It follows that both $o$ and $\bar{p}$ consist of one or more of these five combination sets or classes.

A detailed analysis shows that each encompasses three of the classes with two overlapping, as follows.

Both $o$ and $\bar{p}$ encompass all the illogical persons. In this case, that means $\bar{p} \bar{l} o$.

As regards the remaining four combination sets or classes, it is clear that neither $o$ nor $\bar{p}$ can encompass plo, which is neither of the two.

That leaves the three combination sets or classes plo, $\bar{p} l o$, and $\bar{p} l \bar{o}$. It is easy to see that $o$ and $\bar{p}$ each encompass two of the three classes, but not a third.

Clearly, both include $\bar{p} l o$. What is more, $\bar{p}$ encompasses $\bar{p} l \bar{o}$, but $o$ does not. And $o$ encompasses plo, but $\bar{p} l \bar{o}$ does not.

\section{Generalization of the Syllogism by Means of a Computer Algorithm}

In the syllogisms discussed above, there are three attributes and two premises. But the method should apply equally well to any number of attributes in their present and absent states and any number of premises.

G. Boole proposes certain simplifications with the aim of handling larger numbers of attributes and premises. But in some ways, these simplifications may make the method less perfect. A discussion follows further below.

In any event, what matters here is that, in the end, no type of conceivable simplification can avoid elaborate computations.
Consider the case in which each of the 26 letters of the alphabet stands for an attribute characterizing a class of things. Then let there be 26 premises, alternatively affirmative and negated, as follows: $a$ belongs to $b$; $b$ does not belong to $c$; $c$ belongs to $d$; $d$ does not belong $e$; and so on, ending with $z$ does not belong to $a$.

Then one might seek to establish, for example, what exactly $m$ is in relation to $a, c, e, v, x$, and $z$. Working this out is just about humanly impossible.

There is nothing that G. Boole could have done to avoid laborious computations in this case. The solution is obviously a computer algorithm. It is interesting to note that the solution, namely the computer, owes so much to G. Boole's own algebra. Thus, in the end, his writings did contain the germ of the solution to a problem of which he must have been very much aware. I otherwise do not know whether G. Boole ever considered it possible that a machine might be the answer.

The computer algorithm that is needed should not be all that complex for experienced programmers. But it has never even been attempted. That has much to do with the fact that G. Boole's fundamental formula of Development, namely, $f(x)=f(1) x+f(0)(1-x)$, has generally fallen by the wayside, with the notable exception of the writings of J. Venn.

All the critical steps of the algorithm can be inferred from the mathematical map of Ch. L. Dodgson's two premises discussed in $\S 14.11$.

First, all Equations are redefined in terms of what they deny. Thus, all $a$ belongs to $b$ becomes $a \bar{b}=0$.

Second, all the denials are summed up and equated to 0 , which they in fact together are.

Third, in a step that G. Boole never took but that is much recommended by what one finds in J. Venn, each term is defined in relation to all the other attributes. In the example at hand, there are 24 attributes in addition to $a$ and $b$. That means that the original term $a \bar{b}$ is expanded into $2^{24}$ terms, or $16,777,216$ terms, that are all equal to 0 .

In the example at hand, there are 26 original terms, that is, each can be expanded into $2^{24}$ terms. But that does not mean that there are 436,207,616 $\left(2^{24} \times 26\right)$ terms in total. In fact, the total number of ways in which all 26 terms can be combined in their present and absent states is just $2^{26}$ or $67,108,864$. It goes without saying that the 26 expansions of the 26 original terms overlap.

Fourth, in a step that G. Boole did not take either, all the terms that appear more than once are reduced to just one appearance. After all, in digital mathematics, $x+x=$ $x$. The number of terms that will remain will lie between $2^{24}$ and $2^{26}$.

Fifth, since the aim is to establish what the one attribute $m$ is in relation to the six attributes $a, c, e, v, x$, and $z$, the 19 other attributes need to be eliminated from the 
Equation. As before, G. Boole's method is as follows. For example, to eliminate $b$, one first replaces $b$ by 1 in all the remaining terms and retains the result. This means in effect that all instances of $\bar{b}$ or $(1-b)$ become 0 . Next, one replaces $b$ by 0 in all the remaining terms and retains this result as well. This means in effect that all instances of $\bar{b}$ or $(1-b)$ become 1 . Then one multiplies the two results by multiplying each term of the first result with each term of the second result. When a product of two terms contains both the present state and the absent state of the same attribute, the result is 0 . According to the fundamental axiom of digital mathematics, a thing cannot both have and not have an attribute at the same time. Or, $x \times \bar{x}=0$. These multiplications lead to a great diminution in the number of terms.

All of the new terms contain either $m$ or $\bar{m}$. Both can be factored out as follows:

$$
\begin{aligned}
& (a b c \cdots+\operatorname{def} \cdots+g h i \cdots+\cdots) m \\
& +(j k l \cdots+\text { mno } \cdots+p q r \cdots+\cdots) \bar{m}=0
\end{aligned}
$$

Sixth, (38) can be rearranged as follows. The various elements $a b c . .$. , etc., are all different products of the 25 attributes other than $m$ in either their present state or their absent state. In representing the sum multiplied by $m$ as $A$ and the sum multiplied by $\bar{m}$ as $B,(38)$ can be rewritten in successive steps as

$$
\begin{gathered}
A \times m+B \times \bar{m}=0 \\
A \times m+B+B \times(1-m)=0 \\
A \times m+B-B \times m=0 \\
(A-B) \times m+B=0 \\
(A-B) \times m=-B, \text { and } \\
m=\frac{-B}{A-B}, \text { or also } \\
m=\frac{B}{B-A} .
\end{gathered}
$$

Seventh, (39) has to be developed for all 64 possible combinations of $a, c, e, v, x$, and $z$ in their present and absent states. Each of the 64 combinations will be multiplied by one of the following four Boolean coefficients: $1,0, \frac{0}{0}$, and $\frac{1}{0}$. Of those accompanied by 1 , all are included in $m$. Of those accompanied by $\frac{0}{0}$, none, some, or all are included. Those accompanied by 0 are not equated with 0 . They may or may not exist. But none are taken or included. Those accompanied by $\frac{1}{0}$ do not exist and are therefore to be equated with $0(=0)$.

By means of a computer algorithm designed to execute the above steps, it is possible to do what a human being could not, that is, to define any attribute as part of any number of premises along with any number of other attributes in relation to all the other attributes.

But what about defining two or more attributes in relation to all the other attributes? In a variation on the case presented above, let us assume that not only $m$ but both $m$ and $n$ need to be defined in relation to $a, c, e, v, x$, and $z$, or both $m$ and $n$ for that matter.

G. Boole proposes and illustrates a theorem that applies to defining two or more attributes in relation to all the others in a single statement [62]. It seems possible to expand this theorem to more than one statement or premise. But I refrain from working out the details here. The theorem is discussed again below.

Meanwhile, the desired result can be obtained by first defining $m$ and $n$ separately and then multiplying the results if it is necessary to define all that is both $m$ and $n$ $(m \times n)$ or all that is either $m$ or $n(m+n)$.

In defining $m$ separately, $n$ needs to be eliminated. In defining $n$ separately, $m$ needs to be eliminated.

\section{Conflation of Two Types of Digital Mathematics in G. Boole's Algebra}

Digital mathematics in general is discussed here in a paper on rational human intelligence because the rational activity of the brain runs on digital mathematics, as it were. But there is digital mathematics beyond the digital mathematics by which the brain operates rationally. In other words, part of digital mathematics belongs to the operating system of the rationally functioning brain and part of digital mathematics does not.

Inferring that Socrates is mortal from the two premises 1) that all men are mortal and 2) that Socrates is a man belongs to a type of digital mathematics that is not part of rational human intelligence. It is not necessary to be able to solve all kinds of syllogisms to be a rationally functioning human being. Solving syllogisms is a digital application of rational human intelligence. It is not rational human intelligence itself.

Likewise, the simple arithmetic involved in knowing that one needs to pay $\$ 20$ dollars if one wants two copies of something that costs $\$ 10$ is not part of rational human intelligence. It is an application of rational human intelligence. It is not necessary to be able to count to be a rationally functioning human being.

Inferring that Socrates is mortal from the two said premises and adding 10 to 10 to obtain 20 are both applications of rational human intelligence to mathematical problems. However, the digital mathematics of the former problem is of the same type as the mathematics on which the rational brain runs whereas the quantitative mathematics of the latter problem is not.

For a proper understanding of rational human intelligence, it is necessary to carefully keep the two types of 
digital mathematics apart, the type that constitutes rational human intelligence and the type that does not.

It may be difficult to discern where one type ends and the other begins. In fact, G. Boole totally conflated the two in his "Laws of Thought", the Magna Charta of the Digital Age.

This means in effect that G. Boole is doing two things at the same time in his book while giving every impression that he is only doing one. On the one hand, he is trying to describe how we think. On the other hand, he is trying to describe digital mathematics in general, part of which accounts for how we think.

It is easy to see how this conflation has confused those coming later who have endeavored to interpret G. Boole. $\mathrm{G}$. Boole makes it seem as if he is trying to do one single thing. This fact has encouraged others coming later to try to establish what that one single thing might well be.

As regards what that one single thing might be, there are two candidates: 1) describing how we think and 2) pure mathematics. G. Boole is in fact trying to do both. But precisely because he gives every impression of only doing one thing, there has been a tendency to assume that G. Boole was under the erroneous impression that he did one thing, namely describing how we think, whereas he was in fact doing the other thing, describing digital mathematics.

I rather believe that the search for the single one thing that G. Boole is doing is not necessary. I believe that he is in fact doing both afore-mentioned things. Only, he presents them as one.

\section{Mathematics as Something the Brain Does}

Rational human intelligence is something that the brain does. It is an activity of the brain. It is proposed here that rational human intelligence is in its entirety a mathematical structure of the digital kind. If this proposal is correct, then it follows that the digital mathematics by which the brain operates is an activity of the brain. Digital mathematics is something that the brain does.

But what about the digital mathematics that is not part of rational human intelligence and what about quantitative mathematics?

Inspired in part by G. Boole's efforts to describe rational human intelligence as a mathematical activity of the brain, I have proposed elsewhere that all mathematics can be observed only as an activity of the brain [63]. I refer to this other study for all matter of detail.

In other words, mathematics can only be observed as an empirical phenomenon as something the brain does. It is tempting to think of mathematics as a code inscribed in the great book of nature. And maybe it is. But all the brain has in the end to dissect mathematics is itself and what it empirically does when it does mathematics.
To discover what mathematics really is, the brain needs to rely on itself alone and observe and examine itself because itself is all that it has. The totality of the human experience is how the brain perceives reality outside itself. And that includes mathematics.

In the end, the quest for a deeper understanding of mathematics, including the mathematics of rational human intelligence, is more of a quest to fulfil the famed adage "Know Thyself" than a quest to understand what is outside oneself.

\section{On G. Boole's Use of "Squaring”}

G. Boole has a tendency to seek to expand his digital mathematics by means of certain theorems. The theorems are ingenious and they do validate the overall edifice. But one wonders on occasion whether the added complexity is desirable or needed.

The tendency in question may have to do with a desire to make digital mathematics more like what mathematicians are used to seeing and hence more palatable to them. It is also how G. Boole, as an eminent student of quantitative mathematics, was used to proceeding in quantitative mathematics. Then why not also do the same in digital mathematics?

I have on occasion noticed that there is a temptation to consider what G. Boole does in his "Laws of Thought" to be not quite pure mathematics. At some point in the nineteenth century, mathematics became greatly concerned with the axiomatic approach, that is, with the need to define all the axioms as precisely as possible. In that regard, it was easy to expect resistance to what G. Boole was trying to do [64].

There are two concerns regarding G. Boole's aforementioned theorems. First, they are the result of a desire to simplify complex operations. However, their effectiveness in that regard is limited. In the end, the method needs to accommodate any number of attributes and any number of premises. As the numbers rise, any simplification is soon without effect. At some point, only a computer can deal with all the data where a human being cannot.

Second, the theorems can take the operation farther away from their real life purport than is desirable. As a mathematician, G. Boole was used to dealing in mathematical proofs with intermediate steps of which the interpretation in real life terms is not transparent.

However, J. Venn has done much to show that many of G. Boole's conceptualizations can often be kept much closer to real life purport than G. Boole believed them to be. J. Venn saw no need to keep something abstract if it can be understood in a perfectly commonsensical way. It is possible, however, that G. Boole assumed that the abstract character of the theorems lends the whole edifice more credibility because it makes it look more like what 
mathematicians expect to see.

What has just been said may be illustrated by one of G. Boole's examples. It involves the following two premises derived from elementary geometry [65]:

1) Similar figures consist of all whose corresponding angles are equal and whose corresponding sides are proportional.

2) Triangles whose corresponding angles are equal have their corresponding sides proportional, and vice versa.

What is sought is as follows: How can non-similar figures be defined?

This is the culminating example of G. Boole's treatment of Reduction, which it is itself the culmination of digital mathematics. It is therefore interesting to note that it is possible to take issue with the way in which G. Boole solves the example. lows:

G. Boole represents the attributes symbolically as fol-

$s=$ similar;

$t=$ triangles;

$q=$ having corresponding angles equal; and

$r=$ having corresponding sides proportional.

The two premises may therefore be formulated mathematically as follows:

$$
\begin{aligned}
& s=t r \\
& t q=t r .
\end{aligned}
$$

Equivalents are as follows:

$$
\begin{aligned}
& s-t r=0 ; \\
& t q-t r=0 .
\end{aligned}
$$

The solution that G. Boole obtains is as follows:

$$
\begin{aligned}
1-s= & 0 t q r+2 t q(1-r)+2 \operatorname{tr}(1-q)+t(1-q)(1-r) \\
& +0(1-t) q r+(1-t) q(1-r)+(1-t) r(1-q) \\
& +(1-t)(1-q)(1-r),
\end{aligned}
$$

which can be shortened to

$$
\begin{aligned}
1-s= & t(1-q)(1-r)+(1-t) q(1-r) \\
& +(1-t) r(1-q)+(1-t)(1-q)(1-r),
\end{aligned}
$$

and further to

$$
\bar{s}=t \bar{q} \bar{r}+\bar{t} q \bar{r}+\bar{t} \bar{q}+\bar{t} \bar{q} \bar{r} .
$$

This solution is put into words by G. Boole as follows:

Dissimilar figures consist of all triangles which have not their corresponding angles equal and sides proportional, and of all figures not being triangles which have either their angles equal, and sides not proportional, or their corresponding sides proportional, and angles not equal, or neither their corresponding angles equal nor corresponding sides proportional.

An alternative formulation is as follows:
Dissimilar figures consist of all triangles with corresponding angles equal and corresponding sides proportional and all non-triangles with either corresponding angles equal and corresponding sides not proportional or with corresponding sides proportional and angles not equal or with neither corresponding angles equal nor corresponding sides proportional.

There are two unusual features about G. Boole's solution. First, he squares the left-hand terms in the Equations (40) and (41), as follows:

$$
\begin{aligned}
& (s-t r)^{2}=0 ; \\
& (t q-t r)^{2}=0 .
\end{aligned}
$$

He deems this procedure justified because, in digital mathematics, $x x\left(x^{2}\right)=x$. The results are as follows:

$$
\begin{aligned}
& s+t r-2 s t r=0 ; \\
& t q+t r-t q r=0 .
\end{aligned}
$$

G. Boole assumes that certain sets of premises need to be resolved by squaring and others not. I believe that this distinction makes for a needless complication. A single method that can be applied to all sets of premises seems preferable.

I refrain from repeating all of G. Boole's solution here. While I believe his approach to Reduction and his solutions to be completely valid, $100 \%$ purely mathematical, and fully final, it seems to me that there is still room to make it into a more perfect method.

My two objections to G. Boole's solution of the example at hand concern two characteristics:

1) his solution involves solving different sets of premises in different ways;

2) the symbol "2" as a coefficient of two of the classes in his solution of the above example is defined only as an afterthought.

I therefore propose to solve the same example in a manner that exhibits neither characteristic.

The need is for a single, simple, and universal point of departure of a purely mathematical procedure that applies to any set of premises containing any number of attributes in their present and absent states. The procedure's strictly mathematical character should make it easy to program in computer code. In fact, to have any claim to veracity the procedure needs to be mathematical in the strict sense.

A universal point of departure is presented in the next section. The approach that follows from this point of departure owes most to J. Venn's elucidations of G. Boole's method.

\section{An Alternative to G. Boole's "Squaring": Multiplication by Supplements}

What one seeks to determine at the outset as soon as pos- 
sible is all the classes that do not exist according to the two Equations (40) and (41), that is, all the non-existing combinations of $s$, $t$, and $r$ in their present and absent states according to the first Equation and all the nonexisting combinations of $t, q$, and $r$ in their present and absent states according to the second Equation.

In a universe of thought in which every attribute could combine with every other attribute as properties of a single entity, no rational knowledge would be possible. Exact knowledge is all about which combinations of attributes do not exist.

Equations describe relations between attributes exhibited by entities, such as the relation between the attribute "Paris" and the attribute "the capital of France" in the Equation "Paris $(p)$ is the capital of France (c)" or

$$
p=c \text {. }
$$

It naturally follows that the combinations of the attributes that are being related do exist. In other words, what is both Paris and the capital of France does exist. To convey such existence is the very essence of the Equation.

If any combinations do not exist, then they must somehow involve the supplements, that is, the attributes in their other states, in this case what is not Paris and what is not the capital of France. The two attributes concerned are "Paris" and "the capital of France". The focus is therefore on the combination of the attribute of one side of the Equation with the supplement of the other side of the Equation and the combination of the other side of the Equation with the supplement of the one side of the Equation, that is, the two combinations of what is Paris and not the capital of France or $p(1-c)$ and what is the capital of France and not Paris $(1-p) c$.

How can these two combinations be obtained from Equation (42)?

The first combination is obtained by multiplying $p$ by $(1-c)$ in the digital mathematical sense. The second combination is obtained by multiplying $c$ by $(1-p)$.

However, because $p$ and $c$ are equated with one another, both sides of the Equation need to be multiplied by the same factor. The result is the following two Equations:

$$
\begin{gathered}
p(1-c)=c(1-c) \\
p(1-p)=c(1-p) .
\end{gathered}
$$

In strictly digital mathematical terms, it follows that

$$
p(1-c)=0 \text { and } 0=c(1-p),
$$

and that is because something cannot at the same time exhibit and not exhibit a certain characteristic.

It is always desirable to understand the real life purport of a certain result. In that regard, I find J. Venn's ap- proach to G. Boole's method entirely unobjectionable, whereas G. Boole himself and E. Schröder, together with J. Venn one of the best two interpreters of G. Boole's method, seemed to avoid interpretations in terms of real life purport as much as possible, as if they would make the method less mathematical. I believe that they do not do so in the least.

J. Venn (1834-1923) taught logic as a lecturer in moral science at Cambridge University. After all, in a sense, the study of logic is the study of proper thinking. But it would be inappropriate to contrast J. Venn's approach as non-mathematical with G. Boole's and E. Schröder' as mathematical.

J. Venn was Sixth Wrangler in 1857 at Cambridge University, that is, sixth in rank of the First Class degrees in the Mathematical Tripos. It is clear from passing remarks in the footnotes and elsewhere in his "Symbolic Logic" that he was aware of the most sophisticated advancements in mathematics of his time. There is no doubt that he in the end considered symbolic logic to be as mathematical as G. Boole and E. Schröder would.

In terms of real life purport, in the absence of Equation (42), one does not know whether Paris is or is not the capital of France. "Paris" therefore consists of two parts in relation to "the capital of France": 1) that which is Paris and the capital of France and 2) that which is Paris and not the capital of France.

But because Paris is identified as being exactly equated with the capital of France in (42), it naturally follows that that which is Paris and not the capital of France does not exist. And the same reasoning can be applied to that which is the capital of France and not Paris.

The following general digital mathematical method can be formulated to obtain all the combination sets or classes that constitute rational knowledge, that is, those that do not exist or are equal to 0, for any type of Equation:

Multiply all terms of both sides of the Equation with all the supplements of one side. Then separately multiply all terms of both sides of the Equation with all the supplements of the other side. The two resulting Equations will yield all the non-existent combinations.

Examples of this procedure are provided in the following section.

\section{Examples of Multiplication by Supplements}

20.1. Equations of the Type $x+\frac{0}{0} y=z+\frac{0}{0} w$ in

\section{General}

This is not the place to evaluate how the rule described in the previous section operates in all types of Equations, even if a comprehensive exposition would be useful. 
Suffice it to adduce some examples. G. Boole's example discussed in $\S 18$ involves two Equations. Before returning to this example it will be useful to apply the method of multiplication by supplements to a single Equation that is more complex than either of the two Equations that are part of G. Boole's syllogism discussed in $\S 18$. An example of this single type of Equation is presented in $\$ 20.2$. A second type of single Equation is discussed in $\$ 20.3$.

The first example discussed in the present section concerns a type of Equation that is not discussed by G. Boole, as far as I know, though it is by J. Venn [66].

It is interesting to note that J. Venn misinterpreted the type in the first edition of his "Symbolic Logic" of 1881 [67], as E. Schröder pointed out [68], as a result of which J. Venn corrected his treatment of the Equation in the second edition [69]. The second type is not discussed by anyone, as far as I know. It is a variation on the first type and closely related to it.

The first type is as follows:

$$
x+\frac{0}{0} y=z+\frac{0}{0} w .
$$

It may be put into words as follows:

All $x$ and none, some, or all $y$ are the same as all $z$ and none, some, or all $w$.

In order to obtain all the non-existent classes, those that constitute rational knowledge, one simply applies the two-step rule formulated at the end of $\S 19$.

The first step is to multiply all the terms on both sides of the Equation with the supplements of the left-hand side, namely $(1-x)$ and $(1-y)$. The second step is to multiply all the terms on both sides of the Equation with the supplements of the right-hand side, namely $(1-z)$ and $(1-w)$. The mathematical representation of these two steps is found in the following two Equations:

$$
\begin{aligned}
& x(1-x)(1-y)+\frac{0}{0} y(1-x)(1-y) \\
& =z(1-x)(1-y)+\frac{0}{0} w(1-x)(1-y) \\
& x(1-z)(1-w)+\frac{0}{0} y(1-z)(1-w) \\
& =z(1-z)(1-w)+\frac{0}{0} w(1-z)(1-w)
\end{aligned}
$$

Since the four expressions $x(1-x), y(1-y)$, $z(1-z)$, and $w(1-w)$ are all equal to 0, Equations (44) and (45) can be rewritten as follows:

$$
\begin{aligned}
& 0 \times(1-y)+\frac{0}{0}(1-x) \times 0 \\
& =z(1-x)(1-y)+\frac{0}{0} w(1-x)(1-y)
\end{aligned}
$$

$$
\begin{aligned}
& x(1-z)(1-w)+\frac{0}{0} y(1-z)(1-w) \\
& =0 \times(1-w)+\frac{0}{0}(1-z) \times 0 .
\end{aligned}
$$

And therefore also as follows:

$$
\begin{aligned}
& 0=z(1-x)(1-y)+\frac{0}{0} w(1-x)(1-y) \\
& x(1-z)(1-w)+\frac{0}{0} y(1-z)(1-w)=0 .
\end{aligned}
$$

A short-hand equivalent is as follows:

$$
\begin{aligned}
& z \bar{x} \bar{y}+\frac{0}{0} w \bar{x} \bar{y}=0 ; \\
& x \bar{z} \bar{w}+\frac{0}{0} y \bar{z} \bar{w}=0 .
\end{aligned}
$$

The only two of the four expressions that are equal to 0 are $x \bar{z} \bar{w}$ and $z \bar{x} \bar{y}$. Only an indefinite amount of the two other expressions are equal to 0 . It can therefore not be ascertained whether they are in their entirety equal to 0 .

Each of the two expressions that are equal to 0 consists of two combination sets or classes, for a total of four out of 16 possible cases. The four combination sets or classes equal to 0 are as follows: $\bar{x} \bar{y} z w, \bar{x} \bar{y} z \bar{w}, x y \bar{z} \bar{w}$, and $x \bar{y} \bar{z} \bar{w}$

Equations (44) and (45) reveal in addition that none, some, or all of $\bar{x} \bar{y} w$ and $y \bar{z} \bar{w}$ are non-existent. The four combination sets or classes concerned are $\bar{x} \bar{y} z w$, $\bar{x} \bar{y} \bar{z} w, x y \bar{z} \bar{w}$, and $\bar{x} y \bar{z} \bar{w}$. But two of these four combination sets or classes have already been declared to be equal to 0 , namely $\bar{x} \bar{y} z w$ and $x y \bar{z} \bar{w}$. These two combination sets or classes are therefore once considered in their entirety and once in regard to a totally indefinite amount.

First of all, it is clear that "all" overrides "none, some, or all". The latter leaves open the possibility of "all" and the former confirms "all". Therefore, "all" it is.

Still, the fact that two combination sets or classes are described in two different ways in a single mathematical expression raises questions. Is there a contradiction? The divergence seems somehow unmathematical. One demands greater exactitude from mathematical expressions.

The reason for the ambiguity is that an expression such as

$$
x+\frac{0}{0} y
$$

mimics a linguistic expression that is ambiguous, namely "all $x$ and some $y$ ".

An example is "all freshmen and some band members". In this expression, the freshmen that are band members are signified twice. But it is obvious that all freshmen 
who are band members are included in the overall expression. In other words, the "all" overrides the "none, some, or all".

The mathematical expression in (46) can be rendered more rigorous by defining each term in the expression in relation to all attributes, for example as follows:

$$
x(y+\bar{y})+\frac{0}{0} \bar{x} y
$$

"All $x$, whether $y$ or not $y$, and none, some, or all of $y$, whether it is not $x$ ".

Two different ways of rendering the expression more precise, with two different purports denoting different groups of people as the result, are

$$
x \bar{y}+\frac{0}{0}(\bar{x}+x) y
$$

"All $x$ that is not $y$ and none, some, or all of $y$, whether it is $x$ or not" and

$$
x \bar{y}+\frac{0}{0} \bar{x} y
$$

"All $x$ that is not $y$ and all $y$ that is not $x "$.

Linguistic expressions corresponding to (47), (48), and (49) would be (47) "all the freshmen (whether band members or not) and some band members from among the others", (48) "all the freshmen that are not band members and some band members whether freshmen or not", and (49) "all freshmen except band members along with some band members that are not freshmen".

The rigorous equivalent of the intended purport of the expressions $x+\frac{0}{0} y$ and $z+\frac{0}{0} w$ in Equation (43) is the same as that of (47), not (48) or (49).

\subsection{Equations of the Type $x+\frac{0}{0} y=z+\frac{0}{0} w$ :}

\section{A Concrete Example}

Equation (43) may seem a little abstract. A concrete example may give it some real life dimension.

Consider a track-and-field team at a small college or university in which the following practice is followed: "All 10,000 meter runners $(t)$ and none, some, or all of the 3000 meter steeple chase runners $(s)$ are the same as all the 5000 meter runners $(f)$ and none, some, or all of the marathon runners $(m)$ "'.

In mathematical notation, the desired Equation is as follows:

$$
t+\frac{0}{0} s=f+\frac{0}{0} m
$$

Applying multiplication by supplements, it is immediately obvious that only the following two sets or classes are non-existent or equal to 0 :
1) 5000 meter runners who are neither 10,000 meter runners nor 3000 meter steeple chase runners;

2) 10,000 meter runners who are neither 5000 meter runners nor marathon runners.

Both classes consist of two combination classes. Members of the first category may or may not be marathon runners. Members of the second category may or may not be steeple chase runners.

This much is certain about 5000 meter runners and 10,000 meter runners.

All 5000 meter runners will and must be either 10,000 meter runners or 3000 meter steeple chase runners. Moreover, they may be entirely one or they may be entirely the other. But whatever is the case, all the 10,000 meter runners however many there are, maybe none must be included.

All 10,000 meter runners will and must be either 5000 meter runners or marathon runners. Moreover, they may be entirely one or they may be entirely the other. But whatever is the case, all the 5000 meter runners however many there are, maybe none must be included.

Whereas 5000 and 10,000 meter runners are strictly drawn from two other classes (the former from 3000 meter steeple chase and 10,000 meter runners and the latter from 5000 meter and marathon runners), no such restriction applies to 3000 meter steeple chase and marathon runners.

While 3000 meter steeple chase runners need to be available to run the 5000 meters and marathon runners to run the 10,000 meters, even if none are in the end selected, they are otherwise free to run in any category.

Whereas it seems less likely that 3000 meter steeple chase runners will run in the 10,000 meters or the marathon and that marathon runners will run in the 3000 meter steeple chase or the 5000 meters, the rule expressed in Equation (50) does not keep them from doing so. And they may well wish to run in other races, for example 3000 meter steeple chase runners in, say, the 1500 meters and perhaps even the 800 meters; marathon runners on their part in, say, ultramarathons.

Once it has been established which sets or classes do not exist, it is possible to determine how every attribute in both its present and absent state relates to every other attribute in both its present and absent state. In the present example, this includes defining exactly what 3000 meter steeple chaser runners, 5000 meter runners, 10,000 meter runners, and marathon runners are.

Suffice it to define 5000 meter runners here in terms of the three other attributes.

There are eight possible combination classes involving the three other attributes in Equation (50) in their present and absent states, the following: $t s m, \overline{t s} m, t \bar{s} m, t s \bar{m}$, $\bar{t} \bar{s} m, \bar{t} \bar{s} \bar{m}, t \bar{s} \bar{m}$, and $\bar{t} \bar{s} \bar{m}$. What parts of these eight combination classes make up $f$ ? 
Developing Equation (50) to define $f$ yields the following result. None of the eight combination classes is made equal to 0 . All may or may not exist. Of these eight, two are entirely included in $f$. None, some, or all of four others is also included. And of the two that remain, nothing is included.

Before discussing the real life purport of this result further, it will not be superfluous to detail how the result is obtained in mathematical fashion.

It follows from

$$
t+\frac{0}{0} s=f+\frac{0}{0} m
$$

through multiplication by supplements that

$$
\begin{aligned}
& \overline{t f} \bar{m}=0 ; \\
& \bar{t} \bar{s} f=0 .
\end{aligned}
$$

And, since either of these sets or classes equated with 0 consists of two combination classes, it also follows that

$$
\begin{aligned}
& t s \bar{f} \bar{m}=0 ; \\
& t \bar{s} \bar{f} \bar{m}=0 ; \\
& \bar{t} \bar{s} f m=0 ; \\
& \bar{t} \bar{s} f \bar{m}=0 .
\end{aligned}
$$

These are all the combination sets or classes, among a total of 16 , that are equal to 0 . Accordingly, their sum is also equal to 0 , as follows:

$$
t \bar{s} \bar{f} \bar{m}+t \bar{s} \bar{f} \bar{m}+\bar{t} \bar{s} f m+\bar{t} \bar{s} f \bar{m}=0 .
$$

Equation (51) can be rewritten as

$$
t s(1-f) \bar{m}+t \bar{s}(1-f) \bar{m}+\bar{t} \bar{s} f m+\bar{t} \bar{s} f \bar{m}=0 .
$$

And hence as

$$
t s \bar{m}-t s f \bar{m}+t \bar{s} \bar{m}-t \bar{s} f \bar{m}+\bar{t} \bar{s} f m+\bar{t} \bar{s} f \bar{m}=0 .
$$

It is now possible to define $f$, as follows:

$$
f=\frac{t s \bar{m}+t \bar{s} \bar{m}}{t s \bar{m}+t \bar{s} \bar{m}-\bar{t} \bar{s} m-\bar{t} \bar{s} \bar{m}}
$$

Developing the right-hand side produces the following result:

$$
\begin{aligned}
f= & t s \bar{m}+t \bar{s} \bar{m}+\frac{0}{0} t s m+\frac{0}{0} \bar{t} s m+\frac{0}{0} t \bar{s} m \\
& +\frac{0}{0} \bar{t} \bar{s} \bar{m}-0 \times \bar{t} \bar{s} m-0 \times \bar{t} \bar{s} \bar{m}
\end{aligned}
$$

which is naturally the same as

$$
f=t s \bar{m}+t \bar{s} \bar{m}+\frac{0}{0} t s m+\frac{0}{0} \overline{t s} m+\frac{0}{0} \bar{t} \bar{s} m+\frac{0}{0} \bar{t} \bar{m} .
$$

It will be useful to reflect briefly on the real life pur- port of this last Equation. It appears that two combination classes are fully included in the 5000 meter runners, namely 1) $t s \bar{m}$ " 10,000 meter runners and 3000 meter steeple chase runners who are not marathon runners" and 2) $t \bar{s} \bar{m}$ " 10,000 meter runners who are neither 3000 meter steeple chase runners nor marathon runners".

As regards 1), it follows from the original definition, Equation (50), that 5,000 meter runners and an indefinite amount of marathon runners are made up of 10,000 meter runners and an indefinite amount of 3000 meter steeple chase runners. The members of combination class 1) are at the same time 10,000 meter runners and 3000 meter runners. They therefore absolutely need to be either 5000 meter or marathon runners. At the same time, they are not marathon runners. It follows that they must all be 5000 meter runners.

As regards 2), it follows from the original definition, Equation (50), that 5000 meter runners and an indefinite amount of marathon runners are made up of 10,000 meter runners and an indefinite amount of 3000 meter steeple chase runners. The members of combination class 2 ) are 10,000 meter runners but not 3000 meter steeple chase runners. Still, they are one of the two groups that make up 5000 meter runners and none, some, or all of the marathon runners. However, they are also explicitly not marathon runners. Therefore, they must all be 5000 meter runners.

I refrain from commenting on all the other four combination classes of which an indefinite amount makes up $f$. Suffice it to discuss one of the four, namely tsm "those that are at the same time 10,000 meter runners, 3000 meter steeple chase runners, and marathon runners".

Anyone who is both a 10,000 meter runner and a 3000 meter steeple chase runner, that is, $t s$, must be part of what makes up all the 5000 meter runners and an indefinite amount of the marathon runners.

One, two, or all three of the following three contingents make up set or class $t s: 1)$ those who are 5000 meter runners and only 5000 meter runners $(t s f \bar{m}) ; 2$ ) those who are marathon runners and only marathon runners $(t s \overline{f m})$; 3) those who are both 5000 meter runners and marathon runners (tsmf).

Either of 2) or 3) alone or both together can make up the class $t s m$. If 2) alone does, then none of $t s m$ is $f$. If 3) alone does, then all of tsm is $f$. If both 2) and 3) do, then some of tsm is $f$. In sum, none, some, or all of tsm could be $f$, as Equation (52) states.

The appearance of the minus-sign in (52) for the classes that exist but of which nothing is taken is intriguing. I find it systematically showing up in this case. But nowhere in G. Boole's or J. Venn's works do I find a discussion of the minus-sign. The matter requires further investigation. In pondering the matter, it may be important to consider that, in digital mathematics, the mi- 
nus-sign does not signify the subtraction of a certain quantity as it does in quantity mathematics. It is the opposite of the Boolean operator OR but should not be confused with the NOR function of electrical circuits.

\subsection{The Equation of Type $\frac{0}{0} y=z+\frac{0}{0} w$}

$$
\text { (or } \left.z+\frac{0}{0} w=\frac{0}{0} y\right)
$$

Another type of Equation adduced here to illustrate multiplication by supplements, with the design of obtaining non-existent classes, is discussed neither by G. Boole nor by J. Venn, not in fact by anyone as far as I know.

An example would be as follows:

$$
t+\frac{0}{0} f=\frac{0}{0} m
$$

"All the 10,000 meter runners and none, some, or all of the 5000 meter runners are marathon runners".

In this example, there are three attributes. One way of obtaining another example of this type involves four attributes. It is derived from the example in $\$ 20.2$.

There are two attributes on either side of the Equation in (50). According to the rule formulated above, both sides are multiplied by both supplements of the two terms of the other side. But what if one multiplies a given side by just one supplement of the two terms of the other side? In the following Equation, both sides are multiplied only by the supplement of $x$ instead of by the supplements of both $x$ and $y$ :

$$
x(1-x)+\frac{0}{0} y(1-x)=z(1-x)+\frac{0}{0} w(1-x) .
$$

Since $x(1-x)$ equals 0 , this Equation can be rewritten as

$$
\frac{0}{0} y(1-x)=z(1-x)+\frac{0}{0} w(1-x),
$$

and be put into words as follows:

None, some, or all of $y$ that is not $x$ consists of all $z$ that is not $x$ and none, some, or all of $w$ that is not $x$.

An equivalent formulation is as follows:

All $z$ that is not $x$ and none, some or all of $w$ that is not $x$ are $y$ that is not $x$.

There are four attributes in Equation (50). It is therefore possible to apply four different multiplications by a supplement of an attribute. The four results may be illustrated by means of the example involving a track-andfield team adduced above. They are as follows:

$$
\begin{gathered}
\frac{0}{0} s(1-t)=f(1-t)+\frac{0}{0} m(1-t) \\
t(1-s)=f(1-s)+\frac{0}{0} m(1-s) ;
\end{gathered}
$$

$$
\begin{gathered}
t(1-f)+\frac{0}{0} s(1-f)=\frac{0}{0} m(1-f) ; \text { and } \\
t(1-m)=\frac{0}{0} s(1-m)+m(1-f) .
\end{gathered}
$$

In (50), four combinations class are non-existent, the following:

$$
\begin{aligned}
& t s \bar{f} \bar{m}=0 ; \\
& t \bar{s} \bar{f} \bar{m}=0 ; \\
& \bar{t} \bar{s} f m=0 ; \\
& \bar{t} \bar{s} f \bar{m}=0 .
\end{aligned}
$$

In each of the four Equations (54)-(57), only two combination classes are non-existent, $\bar{t} \bar{s} f m$ and $\bar{t} \bar{s} f \bar{m}$ in (54) and (56) and $t s \bar{f} \bar{m}$ and $t \bar{s} \bar{f} \bar{m}$ in (55) and (57).

The four Equations (54)-(57) are therefore less informative than (50). (54) pairs with (56) and (55) pairs with (57) in terms of the location of $\frac{0}{0}$.

It is (54) and (56) that resemble (53) in structure. And it is the type represented by (53), (54), and (56) that is not discussed by G. Boole nor by J. Venn.

The distinctive characteristic of this type is that a class of the type $\frac{0}{0} f$ stands alone on one side of the Equation but not on the other.

There is more than one way of putting (54)-(57) into words. The study of all the variations is a useful mental exercise that strengthens one's appreciation of what it means to express oneself with great precision. But let it suffice to note only one equivalent for each Equation.

(54) All the 5000 meter runners that are not 10,000 meter runners and none, some, or all of the marathon runners that are not 10,000 meter runners are 3000 meter steeple chase runners that are not 10,000 meter runners.

(55) The 10,000 meter runners that are not 3000 meter steeple runners include all the 5000 meter runners that are not 3000 meter steeple chase runners and none, some, or all of the marathon runners that are not 3000 meter steeple chase runners.

(56) The 10,000 meter runners that are not 5000 meter runners and none, some, or all of the 5000 meter steeple chase runners that are not 5000 meter runners are marathon runners that are not 5000 meter runners.

(57) The 10,000 meter runners that are not marathon runners include all the marathon runners that are not 5000 meter runners and none, some, or all of the 3000 meter steeple chase runners that are not marathon runners.

One mode of abbreviation may be illustrated by the following equivalent of (54): 
(54) Of those not running the 10,000 meter, the 5000 meter runners and none, some, or all of the marathon runners are 3000 meter steeple chase runners.

\subsection{Alternative to G. Boole's Example of "Squaring"}

Equations (40) and (41) can now be solved differently from how G. Boole does, but with the same result. For the sake of brevity, $1-x$ will mostly be written as $\bar{x}$. The need is for determining $1-s$.

1) $s(1-q r)=0 ; 0=\bar{s} q r$ (multiplying both sides twice by the supplement of one the two sides)

$$
\begin{gathered}
s(q r+\bar{q} r+q \bar{r}+\bar{q} \bar{r}-q r) ; 0=\bar{s} q r \\
(1=q r+\bar{q} r+q \bar{r}+\bar{q} \bar{r}) \\
s(\bar{q} r+q \bar{r}+\bar{q} \bar{r}) ; 0=\bar{s} q r(q r-q r=0) \\
s \bar{q} r+s q \bar{r}+s \bar{q} \bar{r} ; 0=\bar{s} q r
\end{gathered}
$$

2) $t q(1-t r)=0 ; \operatorname{tr}(1-t q)=0 \quad$ (multiplying both sides twice by the supplement of one side)

$$
\begin{gathered}
t q-t q t r=0 ; \quad t r-t r t q=0 \\
t q-t q r=0 ; \operatorname{tr}-\operatorname{trq}=0(t t=t) \\
t q(1-r)=0 ; \operatorname{tr}(1-q)=0 \\
t q \bar{r}=0 ; \operatorname{tr} \bar{q}=0
\end{gathered}
$$

3) $s \bar{q} r+s q \bar{r}+s \bar{q} \bar{r}+\bar{s} q r+t q \bar{r}+t \bar{q} r=0 \quad$ (the sum of all that is 0 in 1) and 2) above)
4) $s t \bar{q} r+s \bar{t} \bar{q} r+s t q \bar{r}+s \bar{t} \bar{q}+s t \bar{q} \bar{r}+s \bar{t} \bar{q} \bar{r}$

$$
+\bar{s} t q r+\bar{s} \bar{t} q r+s t q \bar{r}+\bar{s} t q \bar{r}+s t \bar{q} r+\bar{s} t \bar{q} r=0
$$

(defining all the terms for all the attributes; $s \bar{q} r=s t \bar{q} r+s \bar{t} \bar{q} r$, with $t+\bar{t}=1$, and so on)

5) $s t \bar{q} r+s \bar{t} \bar{q} r+s t q \bar{r}+s \bar{t} q \bar{r}+s t \bar{q} \bar{r}+s \bar{t} \bar{q} \bar{r}$

$+\bar{s} t q r+\bar{s} \bar{t} q r+\bar{s} t q \bar{r}+\bar{s} t \bar{q} r=0$

$(s t \bar{q} r+s t \bar{q} r=s t \bar{q} r ; s t q \bar{r}+s t q \bar{r}=s t q \bar{r})$

6) $s t \bar{q} r+s \bar{t} \bar{q} r+s t q \bar{r}+s \bar{t} \bar{q}+s t \bar{q} \bar{r}+s \bar{t} \bar{q} \bar{r}$ $+(1-s) t q r+(1-s) \bar{t} q r+(1-s) t q \bar{r}+(1-s) t \bar{q} r=0$

(switch to explicit and transparent notation)

7) $s t \bar{q} r+s \bar{t} \bar{q} r+s t q \bar{r}+s \bar{t} q \bar{r}+s t \bar{q} \bar{r}+s \bar{t} \bar{q} \bar{r}+t q r$ $-s t q r+\overline{t q} r-s \overline{t q} r+t q \bar{r}-s t q \bar{r}+t \bar{q} r-s t \bar{q} r=0$

8) $s(t \bar{q} r+\bar{t} \bar{q} r+t q \bar{r}+\bar{t} q \bar{r}+t \bar{q} \bar{r}+\bar{t} \bar{q} \bar{r}-t q r$

$$
-\bar{t} q r-t q \bar{r}-t \bar{q} r)=-t q r-\bar{t} q r-t q \bar{r}-t \bar{q} r
$$

9) $s(\bar{t} \bar{q} r+\bar{t} q \bar{r}+t \bar{q} \bar{r}+\bar{t} \bar{q} \bar{r}-t q r-\bar{t} q r)$

$$
=-t q r-\overline{t q} r-t q \bar{r}-t \bar{q} r
$$

$(t \bar{q} r-t \bar{q} r=0 ; t q \bar{r}-t q \bar{r}=0)$

10) $s=\frac{-t q r-\bar{t} q r-t q \bar{r}-t \bar{q} r}{\bar{t} \bar{q} r+\bar{t} q \bar{r}+t \bar{q} \bar{r}+\bar{t} \bar{q} \bar{r}-t q r-\bar{t} q r}$

11) $s=\frac{t q r+\bar{t} q r+t q \bar{r}+t \bar{q} r}{t q r+\bar{t} q r-\bar{t} \bar{q} r-\bar{t} q \bar{r}-t \bar{q} \bar{r}-\bar{t} \bar{q} \bar{r}}$

$$
\text { 12)s } \begin{aligned}
& \frac{1 \times 1 \times 1+0 \times 1 \times 1+1 \times 1 \times 0+0 \times 1 \times 1}{1 \times 1 \times 1+0 \times 1 \times 1-0 \times 0 \times 1-0 \times 1 \times 0-1 \times 0 \times 0-0 \times 0 \times 0} \times t q r \\
& \frac{0 \times 1 \times 1+1 \times 1 \times 1+0 \times 1 \times 0+0 \times 0 \times 1}{0 \times 1 \times 1+1 \times 1 \times 1-1 \times 0 \times 1-1 \times 1 \times 0-0 \times 0 \times 0-1 \times 0 \times 0} \times \bar{t} q \\
& \frac{1 \times 0 \times 1+0 \times 0 \times 1+1 \times 0 \times 0+1 \times 1 \times 1}{1 \times 0 \times 1+0 \times 0 \times 1-0 \times 1 \times 1-0 \times 0 \times 0-1 \times 1 \times 0-0 \times 1 \times 0} \times t \bar{q} r \\
& \frac{1 \times 1 \times 0+0 \times 1 \times 0+1 \times 1 \times 1+1 \times 0 \times 0}{1 \times 1 \times 0+0 \times 1 \times 0-0 \times 0 \times 0-0 \times 1 \times 1-1 \times 0 \times 1-0 \times 0 \times 1} \times t q \bar{r} \\
& \frac{0 \times 0 \times 1+1 \times 0 \times 1+0 \times 0 \times 0+0 \times 1 \times 1}{0 \times 0 \times 1+1 \times 0 \times 1-1 \times 1 \times 1-1 \times 0 \times 0-0 \times 1 \times 0-1 \times 1 \times 0} \times \bar{t} \bar{q} r \\
& \frac{0 \times 1 \times 0+1 \times 1 \times 0+0 \times 1 \times 1+0 \times 0 \times 0}{0 \times 1 \times 0+1 \times 1 \times 0-1 \times 0 \times 0-1 \times 1 \times 1-0 \times 0 \times 1-1 \times 0 \times 1} \times \bar{t} q \bar{r} \\
& \frac{1 \times 0 \times 0+0 \times 0 \times 0+1 \times 0 \times 1+1 \times 1 \times 0}{1 \times 0 \times 0+0 \times 0 \times 0-0 \times 1 \times 0-0 \times 0 \times 1-1 \times 1 \times 1-0 \times 1 \times 1} \times t \bar{q} \bar{r} \\
& \frac{0 \times 0 \times 0+1 \times 0 \times 0+0 \times 0 \times 1+0 \times 1 \times 0}{0 \times 0 \times 0+1 \times 0 \times 0-1 \times 1 \times 0-1 \times 0 \times 1-0 \times 1 \times 1-1 \times 1 \times 1} \times \bar{t} \bar{q} \bar{r}
\end{aligned}
$$




$$
\begin{aligned}
& \text { 13) } s=\frac{1+0+0+0}{1+0-0-0-0-0} \times t q r \\
& +\frac{0+1+0+0}{0+1-0-0-0-0} \times \overline{t q} r \\
& +\frac{0+0+0+1}{0+0-0-0-0-0} \times t \bar{q} r \\
& +\frac{0+0+1+0}{0+0-0-0-0-0} \times t q \bar{r} \\
& +\frac{0+0+0+0}{0+0-1-0-0-0} \times \bar{t} \bar{q} r \\
& +\frac{0+0+0+0}{0+0-0-1-0-0} \times \bar{t} q \bar{r} \\
& +\frac{0+0+0+0}{0+0-0-0-1-0} \times t \bar{q} \bar{r} \\
& +\frac{0+0+0+0}{0+0-0-0-0-1} \times \bar{t} \bar{q} \bar{r} \\
& \text { 14) } s=\frac{1}{1} \times t q r+\frac{1}{1} \times \overline{t q} r+\frac{1}{0} \times t \bar{q} r+\frac{1}{0} \times t q \bar{r} \\
& +\frac{0}{0} \times \bar{t} \bar{q} r+\frac{0}{-1} \times \bar{t} q \bar{r}+\frac{0}{-1} \times t \bar{q} \bar{r}+\frac{0}{-1} \times \bar{t} \bar{q} \bar{r} \\
& \text { 15) } s=t q r+\overline{t q} r-0 \bar{t} \bar{q} r-0 \bar{t} \bar{q} \bar{r}-0 t \bar{q} \bar{r}-0 \bar{t} \bar{q} \bar{r} \text {; }
\end{aligned}
$$

with $t \bar{q} r=0$ and $t q \bar{r}=0$

16) $s=t q r+\overline{t q} r$; with $t \bar{q} r=0$ and $t q \bar{r}=0$

The subtraction of classes in $(o)$ is again intriguing. The classes in question are equal to 0 . It does therefore not make much difference as to whether one adds or substracts them. It should also be noted that -0 is derived, with G. Boole, from $\frac{0}{-1}$. It would need to be further examined what the minus-sign can be traced back to. The matter awaits further investigation.

G. Boole develops for $1-s$, not for $s .1-s$ can be obtained from $(k)$, as follows:

$$
1-s=1-\frac{t q r+\bar{t} q r+t q \bar{r}+t \bar{q} r}{t q r+\bar{t} q r-\bar{t} \bar{q} r-\bar{t} q \bar{r}-t \bar{q} \bar{r}-\bar{t} \bar{q} \bar{r}} .
$$

Therefore

$$
\begin{aligned}
1-s= & \frac{t q r+\bar{t} q r-\bar{t} \bar{q} r-\bar{t} q \bar{r}-t \bar{q} \bar{r}-\bar{t} \bar{q} \bar{r}}{t q r+\bar{t} q r-\bar{t} \bar{q} r-\bar{t} q \bar{r}-t \bar{q} \bar{r}-\bar{t} \bar{q} \bar{r}} \\
& -\frac{t q r+\overline{t q} r+t q \bar{r}+t \bar{q} r}{t q r+\bar{t} q r-\bar{t} \bar{q} r-\bar{t} q \bar{r}-t \bar{q} \bar{r}-\bar{t} \bar{q} \bar{r}} .
\end{aligned}
$$

Or also:

$$
1-s
$$$$
=\frac{t q r+\overline{t q} r-\bar{t} \bar{q} r-\bar{t} q \bar{r}-t \bar{q} \bar{r}-\bar{t} \bar{q} \bar{r}-t q r-\overline{t q} r-t q \bar{r}-t \bar{q} r}{t q r+\overline{t q} r-\bar{t} \bar{q} r-\overline{t q} \bar{r}-t \bar{q} \bar{r}-\bar{t} \bar{q} \bar{r}} \text {. }
$$

And since $t q r-t q r=0$ and $\overline{t q} r-\overline{t q} r=0$,

$$
\begin{aligned}
1-s & =\frac{-\bar{t} \bar{q} r-\bar{t} q \bar{r}-t \bar{q} \bar{r}-\bar{t} \bar{q} \bar{r}-t q \bar{r}-t \bar{q} r}{t q r+\bar{t} q r-\bar{t} \bar{q} r-\bar{t} q \bar{r}-t \bar{q} \bar{r}-\bar{t} \bar{q} \bar{r}} \\
& =\frac{\bar{t} \bar{q} r+\bar{t} q \bar{r}+t \bar{q} \bar{r}+\bar{t} \bar{q} \bar{r}+t q \bar{r}+t \bar{q} r}{\bar{t} \bar{q} r+\bar{t} q \bar{r}+t \bar{q} \bar{r}+\bar{t} \bar{q} \bar{r}-t q r-\bar{t} q r} .
\end{aligned}
$$

However, I believe that this step is unnecessary. $1-s$ can instead be derived from $(p)$ as follows:

$$
1-s=1-(t q r+\overline{t q} r) \text {; with } t \bar{q} r=0 \text { and } t q \bar{r}=0 .
$$

Since there are eight combination classes involving the three attributes $t, q$, and $r$ in their present and absent states, 1 represents all eight. Subtracting two leaves six. Moreover, two do not exist. The result is four combination classes, as follows:

$$
1-s=\bar{t} \bar{q} r+\bar{t} q \bar{r}+t \bar{q} \bar{r}+\bar{t} \bar{q} \bar{r} .
$$

There are no instances of sets of classes defined as $\frac{0}{0}$ ("none, some, or all") in the example at hand. If any had appeared in the development of $s$, they would be likewise specified in the same way in the development of $1-s$. That is because they would have been subtracted from all of the class, which is part of 1 ("all"). Subtracting none, some, or all of a class from all of a class naturally results in none, some, or all of a class.

\section{Critique of E. Schröder's Alternative to}

\section{G. Boole's $\frac{0}{0}$ by Means of a Strictly \\ Digital Example}

The general aim of the examples discussed in the previous section is to illustrate the nature of digital mathematics as one type of mathematics. It is the type of mathematics according to which rational human intelligence operates. A proper appreciation of digital mathematics in general should therefore serve the cause of analyzing rational human intelligence specifically.

One additional, specific aim of the examples was to show that, while G. Boole's method is essentially complete, there may be room for additional streamlining.

The following example, already anticipated in $\$ 14.10$, shares the general aim just stated. But the specific aim is different.

The specific aim is to critique E. Schröder's analysis of that which is represented by the symbol of division in G. Boole's algebra. He saw his approach as a significant modification of G. Boole's method. As far as I can see, this approach constitutes no improvement whatsoever. In fact, it seems rather a setback.

It will be good to begin with a simple example that E. Schröder himself showcases prominently and see how he analyzes it [70]. 
The example revolves around Rappe, which is a German term for a black horse. There is no special word for black horses in English. Accordingly, it is possible to define Rappe ( $r$ ) as all that is both black $(b)$ and a horse (h) and represent it as follows in G. Boole's algebra, as E. Schröder would readily acknowledge:

$$
r=h \times b \text {. }
$$

Or also as follows:

$$
r=h b .
$$

Evidently, in quantitative mathematics, (58) is equivalent to

$$
h=\frac{r}{b} .
$$

If this expression has any meaning in digital mathematics, it ought to describe what horses are in terms of both Rappe (black horse) and black things.

Anyone used to the function of division in quantitative mathematics may wonder what it means that horses are Rappe (black horse) "divided" by black. In digital mathematics, it means that horses are defined as the class that, if it is multiplied by $b$, yields $r$.

Evidently, the division cannot possibly signify what it signifies in quantitative mathematics. In fact, there is complete consensus between G. Boole, and following him, J. Venn, and indeed also E. Schröder [71], that developing $\frac{r}{b}$ according to G. Boole's formula of development unequivocally produces the result that will be presented below.

As has already been noted above, the aim of the formula of development is to determinine what $h$ is in terms of all possible combinations of $r$ and $b$ in both their absent and present states.

The formula of Development applied to the two attributes $r$ and $b$ is as follows:

$$
\begin{aligned}
h= & f(r, b)=f(1,1) \times r b+f(1,0) \times r(1-b) \\
& +f(0,1) \times(1-r) b+f(0,0) \times(1-r)(1-b) .
\end{aligned}
$$

Since

$$
f(r, b)=\frac{r}{b},
$$

$f(1,1), f(1,0), f(0,1)$, and $f(0,0)$ correspond to $\frac{1}{1}(=1), \frac{1}{0}, \frac{0}{1}(=0)$, and $\frac{0}{0}$ respectively. The former can therefore be substituted by the latter in Equation (59), as follows:

$$
\begin{aligned}
f(r, b)= & \frac{1}{1} \times r b+\frac{1}{0} \times r(1-b)+\frac{0}{1} \times(1-r) b \\
& +\frac{0}{0} \times(1-r)(1-b) .
\end{aligned}
$$

An abbreviated version is as follows:

$$
\begin{gathered}
h=r b+\frac{0}{0} \bar{r} \bar{b} ; \text { with } r \bar{b}=0 ; \\
\text { and taking none of } \bar{r} b .
\end{gathered}
$$

Put into words, (60) states that what can be said about horses $(h)$ is that they are what is both Rappe and black $(r b)$ and none, some, or all of what is neither $([1-r][1-b])$.

E. Schröder knows of the type of Equation represented by (60). But he somehow abbreviates (60) into an "unambiguous (eindeutige)" version as follows [72]:

$$
h=r+\bar{b} .
$$

According to E. Schröder, Equation (61) states that horses, as far as one can derive from (58), are "what is either a Rappe (black horse) or not black (was entweder ein Rappe, oder nicht schwarz ist)".

This interpretation is not false. But it is no longer mathematically precise. It does not convey exactly what is known and what is not known. A comparison with (58) and (60) will be useful.

In considering E. Schröder's solution in (61), it will be necessary to consider three versions. It will appear that $\mathrm{E}$. Schröder's symbolic presentation in (61) differs in mathematical terms from the way in which he puts the Equation into words. What is more, there is every reason to assume that E. Schröder's conception of how the Equation needs to be understood differs from both his symbolic interpretation and his interpretation in words.

In what follows, a distinction will therefore be made between

1) Schröder-by-symbols,

2) Schröder-by-words, and

3) Schröder-by-presumed-intent.

As it happens, what is called here Schröder-by-presumed-intent may well be more or less the same in purport as the desired Equation (60), but somewhat inadvertently so.

It may seem somewhat fastidious to discuss examples in some detail. However, concrete examples seem necessary to try to impress on readers the fact that digital mathematics is truly $100 \%$ mathematics. There is every reason to believe that there is a widespread perception that is not quite so or that it is something related to, say, both mathematics and philosophy.

In order to evaluate the three Schröder versions of the relation between $r, b$, and $h$ mentioned above, the need is for knowing exactly which combination classes do not exist, that is, are equal to 0 , in each version.

But first, it will be useful to evaluate Equations (58) and (60), which are in accordance with G. Boole's method. Equation (58) involves four classes that are 0. Equation (60) involves only three. 
Multiplying both sides of Equation (58) by the supplement of one side and then by the supplement of the other side produces the two Equations

$$
r(1-h b)=0 \text { and }(1-r) h b=0 .
$$

According to the second Equation, one class is non-existent.

From the first of the two Equations, it follows that

$$
r(h \bar{b}+\bar{h} b+\bar{h} \bar{b})=0 .
$$

And also that

$$
r h \bar{b}+r \bar{h} b+r \bar{h} \bar{b}=0,
$$

which can also be written as

$$
r h(1-b)+r(1-h) b+r(1-h)(1-b)=0 .
$$

According to this first Equation, three classes are nonexistent, for a total of four in both the first and the second Equations, the following four: $r h \bar{b}, r \bar{h} b, r \bar{h} \bar{b}$, and $\overline{r h b}$.

Equation (60) can also be written as

$$
h=r h+r(1-h)(1-b) .
$$

In this version of the Equation, $h$ is equated in part with $([1-r][1-b])$ instead of with $\frac{0}{0}(1-r)(1-b)$.

Likewise, if Parisian $(p)$ is the same as what is both Parisian and French $(p f)$, then this means that Parisian $(p)$ is also none, some, or all of what is French $\left(\frac{0}{0} r\right)$. One readily imagines "Parisian" as a circle enveloped by "French" as a circle.

The combination classes that are equal to 0 are once again obtained in two steps by first multiplying both sides by the supplement of one side and then by the supplement of the other side, as follows:

$$
\begin{aligned}
\bar{h} h & =\bar{h} r b+\bar{h} h(1-r(1-b)) ; h(1-r b-h[1-r][1-b]) \\
& =(r b+h[1-r][1-b])(1-r b-[1-r][1-b]) .
\end{aligned}
$$

Since nothing can at the same time exhibit and not exhibit a certain term, as in $\bar{h} h$, it follows that

$$
0=\bar{h} r b \text { and } h-h r b-h \bar{r} \bar{b}=0 .
$$

From the second Equation, it follows that

$$
h(1-r b-\bar{r} \bar{b})=0 \text {. }
$$

And since

$$
1=r b+\bar{r} b+r \bar{b}+\bar{r} \bar{b},
$$

Equation (62) can be rewritten as

$$
h(r b+\bar{r} b+r \bar{b}+\bar{r} \bar{b}-r b-\bar{r} \bar{b})=0
$$

and also as

$$
h(\bar{r} b+r \bar{b})=0
$$

and as

$$
h \bar{r} b+h r \bar{b}=0 .
$$

The classes that are equal to 0 in (60) are therefore $\bar{r} h b, r \bar{h} b$, and $r h \bar{b}$. That makes three. In (58), there is a fourth, $r \bar{h} \bar{b}$.

What happened? Clearly, there is a loss of information in the transition from (58) to (60). To what is this loss of information owed?

A simpler example can perhaps make the loss more apparent. The Flemish $(f)$ are all Dutch-speaking $(d)$ Belgians $(b)$ (as contrasted with Walloons, who are Belgians who speak French; there are otherwise Dutch speakers that are not Belgians, namely all the inhabitants of The Netherlands). This statement can be represented by the following Equation:

$$
f=d b .
$$

As in the case of (58), four combination classes are equal to 0 . In (63), they are $f \bar{d} b, f d \bar{b}, f \bar{d} \bar{b}$, and $\bar{f} d b$.

What can be known about Belgians from (63) is as follows:

$$
b=f d+\frac{0}{0} \bar{f} \bar{d} ; \text { with } f \bar{d}=0 .
$$

"The Belgians are all the Flemish, who are evidently Dutch-speaking, and none, some, or all of those that are neither Flemish nor Dutch".

The number of combination classes equal to 0 drops to three, the following: $\bar{f} \bar{d} b, f d \bar{b}$, and $\bar{f} d b$.

This diminution constitutes a decrease in information. The class about which nothing is now no longer known with certainty is $f \bar{d} \bar{b}$ "Flemish that are neither Dutch nor Belgians". The status of this class is clear from the added specification that $f \bar{d}=0$ in (64), but not from the Equation itself.

It is clear from (63) that there is no such thing as Flemings who are not Belgians or not Dutch-speaking. So how did the non-existence of this fourth combination class get lost, as it were, in the transition from (63) to (64), just as a fourth combination class is lost in the transition from (58) to (60)?

It needs to be remembered that (64) is a kind of interpretation of (63). The purpose of Equation (63) is to define $f$ as precisely as possible. Equation (63) precisely and comprehensively defines $f$. It is evident that $f$ is present only and everywhere where both $b$ and $d$ are present. Non- $f(1-f)$ is also fully defined. In a digital system, sharpness of definition means that both a class and its supplement are precisely defined. It follows from (63), that

$$
1-f=1-d b .
$$

And therefore also that 


$$
1-f=d \bar{b}+\bar{d} b+\bar{d} \bar{b} .
$$

By contrast, $b$ is defined in (63) to the extent that it is combined with $d$. In other words, less is known about $b$ (and non- $b$ ) than about $f$.

From the point of view of $b$, Equation (63) states that, when it is combined with $d$, it is equal to $f$. That means that also non- $b$ is defined in relation to $d$ and $f$. However, non- $b$ is not defined in relation to non- $d$. Accordingly, if one considers Equation (63) from the perspective of $b$, it cannot be determined that the class $f \bar{d} \bar{b}$ is equal to 0 .

From the perspective of $f, f$ is always the combination of $d$ and $b$ according to Equation (63). That means among others that it cannot be combined with both non- $d$ and non- $b$ at the same time.

But from the perspective of $b$, the fact that $b$ is $f$ when $b$ is combined with $d$ means that non- $b$ cannot be combined with both $f$ and $d$. But there is no restriction on the combination of non- $b$ with $f$ and non- $d$. It so happens that $b$ is defined in relation to $d$ and not in relation to non- $d$ in (63).

In reality, there is no such thing as $f \bar{d} \bar{b}$, Flemings that are neither Dutch-speaking nor Belgians. All Flemings are Belgians and Dutch-speaking, as Equation (63) states. But this critical fact is lost in (64).

The next step is to analyze what was defined above as

1) Schröder-by-symbols according to Equation (61),

2) Schröder-by-words, and

3) Schröder-by-presumed-intent.

First, Schröder-by-symbols. The complexity instantly increases because there are two ways of interpreting Equation (61), inclusively and exclusively.

E. Schröder obviously interprets it exclusively. $\mathrm{He}$ uses the expression "either... or ... (entweder... oder...)" when putting the Equation into words. However, the exclusive meaning is not conveyed by the Equation itself. It needs to be derived from what is extraneous to the Equation.

It is a fact that a Rappe (black horse) is always black. However, this information is not part of (61). Equation (44) is an abbreviation and hence ambiguous.

If anything, Equation (61) is more easily interpreted inclusively than exclusively. Since $r$ consists of both $r b$ and $r \bar{b}$ and $\bar{b}$ consists of both $r \bar{b}$ and $\bar{r} \bar{b}$, a fully explicit version of (61) is

$$
\begin{aligned}
& h=r b+r \bar{b}+r \bar{b}+\bar{r} \bar{b} \text { and since } \\
& r \bar{b}+r \bar{b}=r \bar{b}, h=r b+r \bar{b}+\bar{r} \bar{b} .
\end{aligned}
$$

The class $h$ encompasses 1) all that is both $r$ and $b, 2$ ) all that is $r$ but not $b$, and 3) all that is neither $r$ nor $b$. The Equation is inclusive because the overlap between $r$ and $\bar{b}$, namely $r \bar{b}$, is included.

By contrast, the unambiguous mathematically exclusive equivalent of (61) is as follows:

$$
h=r b+\bar{r} \bar{b} .
$$

Horses are 1) what is $r$ and also $b$ and 2) what is not $b$ and also not $r$. The Equation is exclusive because the overlap between $r$ and $\bar{b}$, namely $r \bar{b}$, is excluded.

Whether it is understood inclusively or exclusively, Equation (61) signifies something that is entirely different from what E. Schröder assumes that it signifies. There can be no doubt that (61) states that the horses are 1) all that is a Rappe (black horse) and 2) all that is not black.

The equivalent to (61) in relation to the afore-mentioned definition of Belgians would be as follows:

$$
b=f+\bar{d}
$$

This Equation states that the Belgians are 1) all the Flemings and 2) all those who do not speak Dutch. But clearly, the second term following the Equation sign, namely $\bar{d}$ "those who do not speak Dutch" is wrong. There are many people who do not speak Dutch who are not Belgians.

In (65) as in (61), four combination classes are equal to 0 . The empty combination classes pertaining to (61) are $f d \bar{b}, f \bar{d} b, f \bar{d} \bar{b}$, and $\bar{f} d b$. Those pertaining to (65) are $f d \bar{b}, f \bar{d} \bar{d}, f \bar{d} \bar{b}$, and $\bar{f} \bar{d} \bar{b}$. Equations (61) and (65) share only three of these empty combination classes. I refrain from commenting on the real world purport of these classes.

It follows from the above that Schröder-by-words, namely

"Horses are what is either a Rappe or not black" cannot correspond to Schröder-by-symbols. Instead, Schröder-by-words corresponds to

$$
h=\frac{0}{0} r b+\frac{0}{0} \bar{r} \bar{b} .
$$

Schröder-by-words poses its own problems. In Schröder-by-symbols, the second term to the right of the Equation sign was the problem. In Schröder-by-words, it is the first term.

According to Schröder-by-words, whose symbolic representation is (66), there are Rappes that are not horses.

Likewise, the statement "A Belgian is either a Fleming or not a Dutch speaker", which exhibits the same informational structure as (66), does not deny the possibility that there are non-Belgian Flemings.

And yet, there are none, just as there are no Rappes that are not horses. In other words, the combination classes $r \bar{h} b$ and $r \bar{h} \bar{b}$ are equal to 0 .

But Schröder-by-words does not convey this, contrary to what he himself believes.

Equation (66) involves only two classes that are equal to 0 , namely $r h \bar{b}$ and $\bar{r} h b$. These two empty classes are obtained as follows. 
From (66), the following can be derived in sequence:

$$
\begin{gathered}
h=h(r b+\bar{r} \bar{b}) ; \\
h-h(r b+\bar{r} \bar{b})=0 ; \\
h(1-[r b+\bar{r} \bar{b}])=0 ; \\
h(1-r b-\bar{r} \bar{b})=0 ; \\
h(r \bar{b}+\bar{r} b)=0 ; \\
h r \bar{b}+h \bar{r} b=0 ; \text { and } r h \bar{b}+\bar{r} h b=0 .
\end{gathered}
$$

One less class is equal to 0 than when $h$ is determined according to G. Boole's method. That constitutes a diminution in knowledge.

It can be concluded that Schröder-by-words cannot be what E. Schröder intended. According to Schröder-bypresumed-intent, there are no Rappes that are not horses.

E. Schröder's reinterpretation of G. Boole's method involves going to great length to avoid the symbol $\frac{0}{0}$ "none, some, or all". But ironically, what he proposes instead in (66) actually adds an instance of $\frac{0}{0}$ to what would be G. Boole's interpretation. There are now two instances of $\frac{0}{0}$ instead of one.

If the Flemish are the totality of Dutch-speaking Belgians, then what are the Belgians? It is clear that all the Flemish are Belgians, as is the fact that they all speak Dutch. The question therefore arises: What about the non-Flemish? Which of them are Belgians, if any?

Three attributes are at stake, namely "Belgian", "Flemish", and "Dutch-speaking". Consequently, if the aim is to define Belgians in terms of those that are not Flemish, then the focus within the narrow confines of the available attributes is on two classes, 1) any non-Flemings that speak Dutch and 2) any non-Flemings that do not speak Dutch.

From a digital perspective, there is nothing else to be considered than these two classes in light of the available attributes in both their present and absent states. It is clear that there is no information available to deny the existence of either class. Then what can be said about them in terms of Belgians?

It is at this juncture that G. Boole's method offers a distinct advantage by distinguishing between 0 and $\frac{0}{0}$.

Belgians include none, or 0, of 1) the non-Flemings who speak Dutch. But it is not clear about individual members of 2) as to whether they can be included or not. 2) can therefore be characterized as $\frac{0}{0}$.

\section{G. Boole's $\frac{0}{0}$}

\subsection{Three Attemps at Clarification}

Much suspicion has always affected the significance of G. Boole's $\frac{0}{0}$. E. Schröder tried to do entirely without it. It will therefore be useful to give additional definition to $\frac{0}{0}$, and that in three ways, even if additional study and reflection remains desirable.

First, a problem proposed by A. Macfarlane is treated in both digital fashion, including the application of $\frac{0}{0}$, and quantitative fashion in §22.3-5.

Second, a careful distinction will be made in $\$ 23$ between the two meanings that G. Boole assigns to $v$, his symbol for indefiniteness. G. Boole clearly considers them to be equivalent. But they are quite distinct. The resulting confusion has often been interpreted as a handicap of his overall theory of digital mathematics.

Since G. Boole apparently interprets the symbol $\frac{0}{0}$ as being at least in part equivalent to $v$, the confusion pertaining to $v$ is in danger of also affecting the interpretation of $\frac{0}{0}$.

Third, an attempt will be made to add a certain sophistication to the interpretation of $\frac{0}{0}$ in $\$ 24$ by reflecting on the interpretation of $\frac{0}{0} \times \frac{0}{0}$.

\subsection{The Digitality of $\frac{0}{0}$}

But first, it is necessary to emphasize that $\frac{0}{0}$ is digital alright. Again and again, this symbol has been paraphrased above, more or less in the same way as G. Boole does, as "none, some, or all". However, "none", "some", and "all" by all appearance seem to refer to quantity. Does this contradict the notion that $\frac{0}{0}$ is digital? I believe that it does not. Still, the description "none, some, or all" is slightly misleading. It will therefore be desirable to specify further how $\frac{0}{0}$ can be understood digitally.

Digital is all about On (1) and Off (0). How does $\frac{0}{0}$ relate to digital 1 and 0 ? Like 1 and $0, \frac{0}{0}$ describes a set 
or class. The symbol " 1 " describes a set or class of which all members are included. Another way of describing such a set is as follows. If one contemplates any single member of the class, one can be certain that the member is included. The symbol " 0 " describes a set or class of which no members are included. If one contemplates any single member of the class, one can be certain that the member is not included.

In a strictly digital interpretation, $\frac{0}{0}$ describes a class about whose members it cannot be said whether they are included or not. Somewhat provisionally, I prefer to imagine this as follows. If one contemplates any member of the class, one does not know whether it is included (1) or excluded (0). It can be inferred quantitatively that none, some, and all are all possible. But this is an inference. $\frac{0}{0}$ itself is digital.

One might be enclined to argue that $\frac{0}{0}$ cannot be part of a theory of digital mathematics because "none, some, or all" represents neither 0 nor 1 but rather something in between. If this were the case, then digital mathematics would no longer be strictly digital.

However, it should be emphasized that "none, some, or all" is not a description of a third possible state of an attribute in addition to its present state or On-state (1) and its absent state or Off-state (0).

Rather, $\frac{0}{0}$ is a description of our ignorance of which part of a certain set or class exhibits a present state of a certain attribute and which the absent state. It must be either one or the other in a digital system. But it may not be known which of the two it is.

For example, Equation (64) conveys that Belgians include not only all the Flemings but also none, some, or all of the non-Flemings. That does not mean that some Belgians can be both non-Flemish and not non-Flemish at the same time. Nor does it mean that non-Flemings can be both Belgian and not Belgian at the same time. That would be an offense of the fundamental law of digital mathematics.

Clearly, Belgians must be either non-Flemish or not non-Flemish and non-Flemings must be either Belgians or not Belgians. Nothing can be something and not something at the same time.

Equation (64) conveys instead that it is not known which portion of the non-Flemings are Belgians and which portion are not Belgians. In other words, in contemplating a certain member of the non-Flemings, one does not know whether she/he is a Belgian or not.

In the end, as part of rational human intelligence, $\frac{0}{0}$ owes its existence to the fact that rational human intelli- gence is not only about things and events but also about how the brain engages things and events. $\frac{0}{0}$ is not a property of things or events but of the way in which the brain engages things or events, as are in fact in the end also 0 and 1 .

\subsection{Digital $\frac{0}{0}$ and Its Corresponding Quantitative Counterpart}

E. Schröder does seem to comprehend the value of $\frac{0}{0}$ in G. Boole's system. But he describes it as the "general" value. The value that he proposes instead as the crucial value is what he calls the "main" value or "principal" value [73].

The symbol $\frac{0}{0}$ is digital. But there can be a quantitative counterpart. There are a number of mathematical problems in which the digital and the quantitative go hand in hand like the two sides of a coin, both separate and inextricable. These problems are typical in probability theory and I hope to return to them in future installments.

A problem in which determining the quantitative counterpart of $\frac{0}{0}$ plays a crucial role appears in a work on logic by A. Macfarlane. His description of the problem is as follows [74]:

Suppose that of the persons on board a ship which was wrecked, the passengers formed two thirds; and those that were saved in the wreck three-fourths. How many passengers must have been saved, how many lost; how many of the crew must have been saved, how many lost?

A. Macfarlane exhibits a thorough understanding of and great sympathy for-G. Boole's theories. It will be useful to quote his A. Macfarlane's solution in full before proceeding to an alternative. His solution is as follows:

"Let $p$ denote the passengers; $c$ the crew; $s$ saved; $l$ lost.

Then $p+c=1$; or $c=1-p$.

Also $s+l=1$; or $l=1-s$.

And $p=\frac{2}{3} ; s=\frac{3}{4}$.

Now $p s>p+s-1$,

$$
\begin{aligned}
& \text { or }>\frac{2}{3}+\frac{3}{4}-1, \\
& \text { or }>\frac{5}{12} .
\end{aligned}
$$

The passengers saved formed at least five-twelfths.

Since the limit of $(1-p)(1-s)$ is the same as that for $p s$ but with a contrary sign: 


$$
(1-p)(1-s)>-\frac{5}{12}
$$

The crew lost may have been none.

Again. $(1-p) s>-p+s$,

$$
\begin{aligned}
& \text { or }>-\frac{2}{3}+\frac{3}{4}, \\
& \text { or }>\frac{1}{12} .
\end{aligned}
$$

The crew saved formed at least one-twelfth.

Since the limit $p(1-s)$ is the same as that for $(1-p) s$ but taken with the contrary sign,

$$
p(1-s)>-\frac{1}{12} \text {. }
$$

The passengers lost may have been none".

The sign $\geq$ is probably preferable over $>$. But this is not the place to examine A. Macfarlane's analysis among possible analyses.

An expression such as

$$
p+s-1
$$

represents A. Macfarlane's approach. It is clear that, if $p s$, that is, the overlap of $p$ and $s$, is equal to 0 , then the sum of $\frac{2}{3}$ and $\frac{3}{4}$ will overshoot 1 by a certain amount, namely $p+s-1$. The overlap $p s$ needs therefore to be at least as large as the overage for $p+s$ to be 1 or smaller than 1 .

However, what would be desirable is a single systematic, comprehensive approach.

In developing such a comprehensive algorithm, it is useful to imagine $p$ and $s$ as two circles whose size is $\frac{2}{3}$ and $\frac{3}{4}$ respectively, as follows:

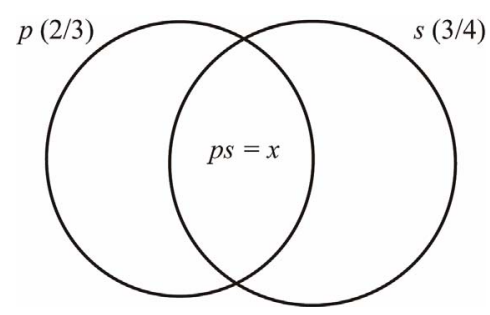

There are two inextricable facets to the problem, a digital side and a quantitative side. The digital side is not considered by A. Macfarlane. It will therefore be useful to begin with this side. It involves the use of the symbol $\frac{0}{0}$.

Suppose that what is sought, that is, $x$, is the number of passengers who have been saved (ps)? The answer is represented by the overlap area of the two circles in the
Venn diagram above. The following Equation applies:

$$
x=p s .
$$

The need is for determining $x$. It is not possible to determine $x$ instantly from Equation (67).

It does follow, however, from (67) that

$$
\begin{gathered}
p=\frac{x}{s} \text { and that } \\
s=\frac{x}{p} .
\end{gathered}
$$

The Venn diagram above already offers a certain intuitive grasp of the problem.

If one tries to determine $p$, then the difference between $p$, all the passengers, and $p s$, the saved passengers, will be a certain portion of $1-s$, that is, of those that are not saved, more specifically the non-saved passengers.

A look at the Venn diagram above makes this fact more transparent. It so happens that the sizes of $p$ and $1-s$ are known, namely $\frac{2}{3}$ and $1-\frac{3}{4}=\frac{1}{4}$.

Alternatively, if one tries to determine $s$, then the difference between $s$, the saved persons, and $p s$, the saved passengers, will be a certain portion of $1-p$, that is, of those that are not passengers, more specifically the saved non-passengers.

A look at the Venn diagram presented above makes this fact too more transparent. It so happens that the sizes of $s$ and $1-p$ are known, namely $\frac{3}{4}$ and $1-\frac{2}{3}=\frac{1}{3}$.

It is a fact that $p s$, or that which is both $p$ and $s$, cannot be larger than either $p$ or $s$. In addition, it is a fact that $p s$ cannot be larger than the smallest of either $p$ or $s$, which happens to be $p$.

The digital solution to the problem can be obtained through G. Boole's formula of development. According to this formula, (68) is equal to

$$
\begin{aligned}
p= & \frac{1}{1} \times x s+\frac{1}{0} \times x(1-s)+\frac{0}{1} \times(1-x) s \\
& +\frac{0}{0} \times(1-x)(1-s),
\end{aligned}
$$

or also to

$$
p=x s+\frac{0}{0}(1-x)(1-s) ; \text { with } x(1-s)=0 .
$$

Equation (70) can be abbreviated because $x$ is part of $s$ and $1-s$ is part of $(1-x)(1-s)$, as the Venn diagram above illustrates. What is both $x$ and $s$ is therefore the same as what is just $x$ and what is both $1-s$ and $(1-x)(1-s)$ is the same as what is just $1-s$. The abbreviation in question is therefore as follows:

$$
p=x+\frac{0}{0}(1-s) \text {. }
$$


It follows from (71) that

$$
p-\frac{0}{0}(1-s)=x .
$$

As regards the quantitative facet of the problem, it appears that the sizes of both $p$ and $1-s$ are known.

Equation (72) can therefore also be presented as

$$
\frac{2}{3}-\frac{0}{0} \times \frac{1}{4}=x(=p s) \text {. }
$$

This Equation conveys that, in order to obtain the quantity of $p s$ "saved passengers", one subtracts "none, some, or all" $\left(\frac{0}{0}\right)$ of $\frac{1}{4}$ from $\frac{2}{3}$. In other words, one can subtract as little as 0 and as much as $\frac{1}{4}$.

It would therefore appear that the quantity of saved passengers can be as many as $\frac{2}{3}-0$ or $\frac{2}{3}$ and as few as $\frac{2}{3}-\frac{1}{4}$ or $\frac{5}{12}$ of the people on the ship, as well as any quantity in between.

Accordingly, as many as all the passengers may have been saved and at least $\frac{5}{8}$ of them (or $\frac{5}{12}: \frac{2}{3}$ ) must have been, that is, about $62.5 \%$.

In this case, the minimum of 0 and the maximum of $\frac{1}{4}$ derived from the digital solution of the problem are the actual minimum and the actual maximum. However, this is not always the case. The actual minimum may be higher and the actual maximum may be lower.

The interpretation of the quantitative counterpart of $\frac{0}{0}$ will therefore need to be further refined. This is done in $\$ 22.4$ below.

E. Schröder's so-called "unambiguous (eindeutige)" version of (71) would be

$$
p=x+(1-s),
$$

that is, (71) after removing $\frac{0}{0}$.

Accordingly, it would follow from (73) that $p-(1-s)=x$, and therefore that

$$
\frac{2}{3}-\frac{1}{4}=\frac{5}{12}=x \text {. }
$$

It is true that no fewer than $\frac{5}{12}$ of all the people on the ship, or at least $\frac{5}{8}$ of all the passengers, have been saved. But the number $\frac{5}{8}$ serves as a lower limit. It is no less likely that, say, $\frac{6}{8}$ or $\frac{7}{8}$ of the passengers have been saved. These possibilities seem to get lost in E. Schröder's approach.

E. Schröder seems to view his abbreviation of G. Boole's approach as a gain in precision. But it is rather a loss. A single number may seem to be more precise than a range of numbers. However, in the present case, it is a range that describes the mathematical reality precisely, not a single number. It is the function of the symbol $\frac{0}{0}$ to denote this range with mathematical precision.

\subsection{Determining the Quantitative}

\section{Counterparts of $\frac{0}{0}$}

In the case discussed in $\$ 22.3$, namely determining $p s$ "saved passengers", a range of the quantity corresponding to $\frac{0}{0}$ is obtained. The range of $p s$ is $\frac{1}{4}$, from a minimum of 0 to a maximum of $\frac{1}{4}$.

But the minimum may be higher and the maximum lower and the range hence shorter. There are three possible cases: 1) either the maximum is lower; 2) or the minimum is higher; 3 ) or both are the case.

For example, in determining $p l$ "lost passengers", both the minimum is higher and the maximum is lower. The equivalent to Equation (71) above pertaining to the solution of $p s$ is as follows:

$$
p=x+\frac{0}{0}(1-l) .
$$

Along the same lines, the equivalent of Equation (72) pertaining to the solution of $p s$ is the following:

$$
p-\frac{0}{0}(1-l)=x .
$$

It appears that the sizes of both $p$ and $1-l$ are known.

Equation (74) can therefore also be presented as

$$
\frac{2}{3}-\frac{0}{0} \times \frac{3}{4}=x(=p l) \text {. }
$$

At face value, Equation (75) conveys that, in order to obtain $p l$ "lost passengers", one subtracts an indefinite amount $\left(\frac{0}{0}\right)$ of a class with size $\frac{3}{4}$ from $\frac{2}{3}$. Naturally, it is not possible to subtract the entire class.

The range of $\frac{3}{4}$ extends from 0 to $\frac{3}{4}$. However, the minimum of 0 is raised to $\frac{5}{12}$ and the maximum of $\frac{3}{4}$ 
is lowered to $\frac{2}{3}$. Accordingly, the actual range from $\frac{5}{12}$ to $\frac{2}{3}$ is $\frac{1}{4}$, exactly what it was in the case of determining $p s$.

The need is for a uniform method to determine the actual minimum, whether it is raised or not, and the maximum, whether it is lowered or not, of the range of the class characterized digitally as $\frac{0}{0}$. Such a method is described in the next section.

\subsection{Determining Minimum and Maximum of Quantities Corresponding to Digital $\frac{\mathbf{0}}{\mathbf{0}}$}

In Equation (72), $x$ is defined in terms of $p$ and $1-s$. In Equation (74), $x$ is defined in terms of $p$ and $1-l$. The ranges characterized by $\frac{0}{0}$ are $1-s$ and $1-l$ respectively.

The actual minimum and the actual maximum of the ranges in question, whether curtailed or not, can be derived respectively from the digital sum of $p$ and $1-s$ or $1-l$ and the digital product of $p$ and $1-s$ or $1-l$.

The minimum is the amount by which the sum is larger than 1 or $100 \%$. If the sum is not larger than 1 , then the actual minimum is 0 and therefore not raised.

Together, two sets or classes evidently cannot be larger than 1 or $100 \%$ or everything that exists. Therefore, if their sum is larger than 1, then that is because they overlap at least by the amount that their sum exceeds 1 or $100 \%$.

In Equation (72), the sum of $p$ and $1-s$ is

$\frac{2}{3}+\frac{1}{4}=\frac{11}{12}$. The sum is less than 1 . Accordingly, the minimum of 0 is not raised.

In Equation (74), the sum of $p$ and $1-l$ is $\frac{2}{3}+\frac{3}{4}=\frac{17}{12}$. This sum does exceed 1 by $\frac{5}{12}$. Accordingly, the minimum of $\frac{0}{0}$ is raised from 0 to $\frac{5}{12}$.

What is the real life purport of the manner in which the minimum is obtained? Consider Equation (72):

$$
p-\frac{0}{0}(1-s)=x .
$$

In this Equation, saved passengers ( $x$ or $p s$ ) are defined as that which is left when one subtracts from all the passengers $(p)$ an indefinite amount $\left(\frac{0}{0}\right)$ of those that are not saved $(1-s)$.
Naturally, the non-saved persons $(1-s)$ that are being subtracted from the passengers need to be non-saved passengers. One cannot subtract non-passengers from passengers.

But the key point is this. If a certain minimum number of passengers are not saved, then these non-saved passengers will reduce the number of those among the passengers who are saved at least by that minimal number.

That minimum number will also be the lowest limit of the quantity of the class characterized digitally as $\frac{0}{0}$, which in this case characterizes those who need to be subtracted from the passengers in order to obtain the saved passengers.

There will be a minimal amount of non-saved passengers to the extent that the passengers and the non-saved persons minimally overlap. The overlap evidently is made up of the non-saved passengers.

There will be an overlap if the sum of passengers and non-saved persons is more than $100 \%$ or all the people. That means that some must be both passengers and nonsaved persons at the same time.

The amount by which their sum is more than $100 \%$ constitutes the minimal overlap. The overlap may obviously be larger.

In Equation (72), $p$ is $\frac{2}{3}$ and $1-s$ is $\frac{1}{4}$. The sum is $\frac{2}{3}+\frac{1}{4}=\frac{11}{12}$. There is therefore no necessary minimal overlap. Accordingly, $p$ can be reduced by as little as 0 to obtain $p s$. The minimum is therefore 0 .

In Equation (74), $p$ is $\frac{2}{3}$ and $1-l$ is $\frac{3}{4}$. The sum is $\frac{2}{3}+\frac{3}{4}=\frac{17}{12}$. The minimal overlap is therefore $\frac{5}{12}$. Accordingly, $p$ needs to be reduced minimally by that amount. The minimum is therefore $\frac{5}{12}$.

Next is determining whether the maximum of the quantitative range of the class characterized digitally as $\frac{0}{0}$ needs to be lowered and, if so, by how much. $\frac{0}{0}$ characterizes digitally what needs to be substracted in Equations (72) and (74). What is subtracted is $p(1-s)$ and $p(1-l)$ respectively.

It is self-evident that what needs to be subtracted cannot be more than the maximum size of $p(1-s)$ or $p(1-l)$ respectively. The maximum size of these two digital products is the same as the size of the smallest of the two sets or classes in each product.

In other words, if there are 5 doctors $(d)$ and 10 Frenchmen $(f)$, the maximum number of French doctors 
$(f d)$ is 5 , when the overlap between the two is maximal.

What is the real life purport of this? It will be useful to return once again to Equation (72), namely

$$
p-\frac{0}{0}(1-s)=x \text {. }
$$

In this Equation, saved passengers ( $x$ or $p s$ ) are defined as that which is left when one subtracts from all the passengers $(p)$ an indefinite amount $\left(\frac{0}{0}\right)$ of those that are not saved $(1-s)$.

The non-saved persons in question ought to be all passengers. But there can be no more non-saved passengers to subtract from the passengers than there are nonsaved passengers.

Nor can there be more non-saved passengers to subtract from the non-saved passengers than there are nonsaved persons.

Furthermore, there can be no more non-saved passengers than those that are both non-saved persons and passengers at the same time.

If the maximum number of non-saved passengers is lower than the maximum of the range of the class characterized digitally as $\frac{0}{0}$, then the range needs to be lowered accordingly.

In Equation (72), $p$ is $\frac{2}{3}$ and $1-s$ is $\frac{1}{4}$. The maximum size of the digital product is therefore $\frac{1}{4}$. This is not less than $\frac{1}{4}$. Accordingly, the maximum does not need to be lowered.

In Equation (74), $p$ is $\frac{2}{3}$ and $1-l$ is $\frac{3}{4}$. The maximum size of the digital product is therefore $\frac{2}{3}$. This is less than the maximum $\frac{3}{4}$. Accordingly, the maximum needs to be reduced from $\frac{3}{4}$ to $\frac{2}{3}$.

Along these lines, a complete digital-quantitative mathematical map of A. Macfarlane's example can be developed. The need is for determining four categories in total, as follows: $p s, p l, c s$, and $c l$.

There are two ways of determining each of the four categories, for a total of eight ways.

In two of the eight ways, neither the maximum nor the minimum are changed, namely in 1) and 5) below. In two cases, the minimum is raised, namely in 2) and 6) below. In two cases, the maximum is lowered, namely in 4) and 8 ) below. And in two cases, both the minimum is raised and the maximum lowered, namely in 3) and 7) below.

The eight ways involve the following eight Equations, two of which have already been mentioned above, namely 1) and 3):

1) $p-\frac{0}{0} \times(1-s)=x=p s$;

2) $s-\frac{0}{0} \times(1-p)=x=p s$;

3) $p-\frac{0}{0} \times(1-l)=x=p l$;

4) $l-\frac{0}{0} \times(1-p)=x=p l$;

5) $c-\frac{0}{0} \times(1-s)=x=c s$;

6) $s-\frac{0}{0} \times(1-c)=x=c s$;

7) $c-\frac{0}{0} \times(1-l)=x=c l$; and

8) $l-\frac{0}{0} \times(1-c)=x=c l$.

The relevant quantities are as follows:

1) $\frac{2}{3}-\frac{0}{0} \times \frac{1}{4}=p s$. Range of (class characterized digitally as) $\frac{0}{0}$ : from 0 to $\frac{1}{4}$.

Result: $\frac{5}{12} \leq p s \leq \frac{2}{3}$.

2) $\frac{3}{4}-\frac{0}{0} \times \frac{1}{3}=p s$. Range of $\frac{0}{0}$ : from $\frac{1}{12}$ to $\frac{1}{3}$.

Result: $\frac{5}{12} \leq p s \leq \frac{2}{3}$.

3) $\frac{2}{3}-\frac{0}{0} \times \frac{3}{4}=p l$. Range of $\frac{0}{0}$ : from $\frac{5}{12}$ to $\frac{2}{3}$.

Result: $0 \leq p l \leq \frac{1}{4}$.

4) $\frac{1}{4}-\frac{0}{0} \times \frac{1}{3}=p l$. Range of $\frac{0}{0}$ : from 0 to $\frac{1}{4}$.

Result: $0 \leq p l \leq \frac{1}{4}$.

5) $\frac{1}{3}-\frac{0}{0} \times \frac{1}{4}=c s$. Range of $\frac{0}{0}$ : from 0 to $\frac{1}{4}$.

Result: $\frac{1}{12} \leq c s \leq \frac{1}{3}$.

6) $\frac{3}{4}-\frac{0}{0} \times \frac{2}{3}=c s$. Range of $\frac{0}{0}$ : from $\frac{5}{12}$ to $\frac{2}{3}$.

Result: $\frac{1}{12} \leq c s \leq \frac{1}{3}$. 
7) $\frac{1}{3}-\frac{0}{0} \times \frac{3}{4}=\mathrm{cl}$. Range of $\frac{0}{0}$ : from $\frac{1}{12}$ to $\frac{1}{3}$.

Result: $0 \leq c l \leq \frac{1}{4}$.

8) $\frac{1}{4}-\frac{0}{0} \times \frac{2}{3}=c l$. Range of $\frac{0}{0}$ : from 0 to $\frac{1}{4}$.

Result: $0 \leq c l \leq \frac{1}{4}$.

The actual range of the classes characterized digitally by $\frac{0}{0}$, including any possible raising of the minimum or lowering of the maximum, is in all cases $\frac{1}{4}$. This is the size of the smallest possible class, the lost persons. It is the smallest wiggle space, as it were, in the relations between the classes and their possible overlaps.

These results are naturally the same as A. Macfarlane's. But they are obtained through a single consistent algorithm that provides a complete mathematical map of the problem and that could easily be converted into a computer program.

I delay until a later occasion the transposal of examples like this to the realm of probability theory. The result would be the introduction of ranges of probability into probability theory.

For example, one might ask the following question: If one has an acquaintance on the boat of A. Macfarlane's example who is a passenger, what is the chance that she or he has survived? It is between $62.5 \%$ and $100 \%$. Suppose one were offered to receive $\$ 1000$ if the passenger survives. How much should one pay for the privilege of entering the bet on fair conditions? The sum is $\$ 812.5$ because one has on average a chance of $81.25 \%$, that is, halfway between $62.5 \%$ and $100 \%$, that the passenger has survived. This is the best one can do considering the data.

\section{The Confusing Treatment of the Indefinite Symbols $v$ and $\frac{0}{0}$ in G.} Boole's Works

\subsection{The Distinct Mathematical Definitions of 1) $v$ and 2) $\frac{0}{0}$}

The design of $\S 21$ and $\S 22$ was to illustrate and clarify the full mathematical validity of G. Boole's $\frac{0}{0}$ "none, some, or all".

Those who are familiar with G. Boole's writings will be aware that he very often uses a symbol $v$ and considers the function of this symbol to be generally equivalent to $\frac{0}{0}$.

It is also an undeniable fact that he defines $v$ in two distinct and contradictory ways, as follows:

1) "all, some, or none" [75];

2) "some... [or] all" [76].

The result is two symbols, namely 1) $v$ and 2) $\frac{0}{0}$, and two definitions.

It is one thing that the symbol $\frac{0}{0}$, which looks to the quantitative mathematician like a fraction in which the numerator and the denominator are both zero, has been viewed with great suspicion.

But it has not helped the cause of $\frac{0}{0}$ that G. Boole viewed it as being equivalent to $v$, a symbol that he defines in two different ways.

G. Boole has been criticized for his use of the symbol $v$ to about the same degree as his use of $\frac{0}{0}$ has been considered suspect.

But there is yet an additional complication, which only seems to incrase the confusion. As will be noted in $\S 23.2$, it is eminently probable that G. Boole's symbol $v$ originates in the use of "some" in the so-called particular statements of Aristotelian and scholastic logic. But there is a problem. In Aristotelian and scholastic logic, "some" excludes both "none" and "all". This is a definition that G. Boole nowhere assigns to $v$. As was observed above, one finds it defined as "some or all".

There is no denying that the whole matter is confusing. Still, I believe that the confusion does not handicap G. Boole's method and system in any fundamental way.

What is more, it appears to me definitely possible to clear up the confusion and thereby tie up a loose end in $\mathrm{G}$. Boole's method in rigorously mathematical terms.

This is not the place to treat the matter at length. May it suffice to note that one approach that I find acceptable would be to eliminate $v$ altogether and to define $\frac{0}{0}$ strictly digitally, as has been done above, as a marker of classes regarding whose individual members one does not know whether they are included (1) or excluded (0).

If one does want to retain $v$, then one ought to differentiate it from $\frac{0}{0}$, it seems to me. Why simultaneously use two symbols with the exact same meaning?

I see an opportunity to use $v$ as an equivalent of "some", excluding both "none" and "all", more or less in the same meaning in which it is used in so-called particular statements. In that regard, $\frac{0}{0}$ and $v$ would belong 
to radically different components of rational human intelligence in that $\frac{0}{0}$ would be digital and $v$ quantitative. I hope to present a more detailed treatment of this matter elsewhere.

\subsection{The Origin of G. Boole's $v$}

Upon closer inspection, it is easily possible to detect the origin of G. Boole's $v$. It can be traced back to the "some" of the so-called particular statements of Aristotelian and scholastic logic. This "some" excludes both "none" and "all". But as has already been noted in the previous section, G. Boole nowhere defines $v$ in this way, only adding to the confusion characterizing his use of $\frac{0}{0}$ and $v$. One only finds the definition "some or all" in his work, in addition to "none, some, or all".

Particular statements are of the two types "Some Xs are Ys" and "Some Xs are not Ys". They are traditionally signified by the letters $\mathrm{I}$ and $\mathrm{O}$ respectively.

Particular statements are opposed to the so-called universal statements of the types "All Xs are Ys" and "No $\mathrm{Xs}$ are Ys", which are signified by the letters A and I respectively.

The letters A, I, E, and $\mathrm{O}$ are taken from the Latin words affirmo "I affirm" and nego "I deny".

That the particular statements are the origin of $\mathrm{G}$. Boole's $v$ is even more apparent from his earlier "The Mathematical Analysis of Logic" of 1847 [77] than from his later "An Investigation of the Laws of Thought" of 1854.

I hope to lay out in detail in a future paper how the particular statements of Aristotelian and scholastic logic are an expression of an entirely different component of rational human intelligence than the universal statements of that same logic. I have been much inspired in reaching this conclusion by the writings of J. Venn.

Accordingly, there is something deeply tortured in Aristotelian and scholastic logic when it comes to treating particular statements together with universal statements as members of one and the same species.

This approach has dominated logic for two and a half millennia. It has affected G. Boole's strictly mathematical theory of logic. And it has especially created confusion in regard to his employment of the two symbols $\frac{0}{0}$ and $v$, and especially of the latter.

It is time to separate the types A and E from the types I and $\mathrm{O}$ and treat them as entirely different facets of rational human intelligence.

In the end, one does wonder why G. Boole did not extract himself entirely from the Aristotelian and scholastic particular statements.
One can only surmise that the Aristotelian system and the particular statements that are part of it appeared even to him as too hallowed by history to allow for a complete departure.

But the consequence in the end is what I believe to be two shortcomings in G. Boole's system.

The first shortcoming concerns the incorporation of particular statements into the same system as universal statements, into which they do not really fit.

The second shortcoming is the absence of an appreciation of the very specific role that particular statements do otherwise play in the totality of rational human intelligence. This role will be detailed elsewhere.

\section{On the Multiplication of Coefficients}

To articulate the relevance of $\frac{0}{0}$ and the other coefficients, one more illustration of their purport may be considered. This illustration concerns the (digital) multiplication of the coefficients.

Interpreters have struggled mightily with the interpretation of $\frac{0}{0}$. It may therefore seem precocious to consider the purport of the expression $\frac{0}{0} \times \frac{0}{0}$.

G. Boole did not devote any attention to the possibility of multiplying coefficients, as far as I know. But there is a footnote in J. Venn's "Symbolic Logic" to the effect that

$$
\frac{0}{0} \times \frac{0}{0}=\frac{0}{0}
$$

and that in the sense that "our ignorance is equally complete before and after the multiplication" [78].

The following discussion of the multiplication of coefficients is otherwise designed as an extension of $G$. Boole's and J. Venn's ideas.

There are four coefficients in G. Boole's system, namely $0,1, \frac{0}{0}$, and $\frac{1}{0}$.

The coefficient $\frac{1}{0}$ can readily be eliminated from consideration when it comes to multiplication because it accompanies classes that do not exist. That leaves 0,1 , and $\frac{0}{0}$.

These three coefficients can be multiplied with one another in six ways, the following:

$$
0 \times 0 ; 0 \times \frac{0}{0} ; 0 \times 1 ; \frac{0}{0} \times 1 ; 1 \times 1 ; \text { and } \frac{0}{0} \times \frac{0}{0} \text {. }
$$

To clarify the purport of multiplied coefficients, a problem proposed by G. Boole may be solved in what 
follows in a manner that differs from the manner in which G. Boole solves it.

What is more, this alternative solution involves the multiplication, in the digital sense naturally, of coefficients.

The problem involves the following definition of wealth:

Wealth ( $w)$ consists of things transferable $(t)$, limited in supply ( $s)$, and either productive of pleasure $(p)$ or preventive of pain $(r)$.

This statement can be rendered as follows in G. Boole's algebra:

$$
w=s t[p+r(1-p)] .
$$

In relation to Equation (76), G. Boole proposes to establish what the things both transferable $(t)$ and productive of pleasure $(p)$, that is, $t \times p$ or $t p$, are in relation to the three other attributes $w, s$, and $r$.

In this connection, he proposes a sophisticated method in which he equates $t p$ with $y$ in a separate Equation. He then brings terms to the same side in both this Equation and Equation (76) and squares the terms joined to the same side and equates their sum to zero. The details of this procedure exceed the scope of the present paper.

I do wonder, however, whether the alternative procedure to be proposed in what follows is longer than G. Boole's.

I instead propose, (1), to eliminate $t$ from (76) and obtain what $p$ is in relation to $w, s$, and $r$ and, (2), to eliminate $p$ from (76) and obtain what $t$ is in relation to $w, s$, and $r$.

As was noted before, elimination of $p$ involves equating $p$ first with 1 and then with 0 and equating the product of the two with zero; and likewise for eliminating 1. I refrain from laying out all the details. The two following Equations are obtained:

$$
\begin{gathered}
t=\frac{w s r+w \bar{s} r+w s \bar{r}+w \bar{s} \bar{r}}{w s r+w s \bar{r}-\bar{w} s r} ; \\
p=\frac{w \bar{s} r+w s \bar{r}+w \bar{s} \bar{r}}{w s \bar{r}} .
\end{gathered}
$$

The next step is to develop the right-hand sides of both (77) and (78). The result is as follows:

$$
\begin{aligned}
t= & 1 \times w s r+1 \times w s \bar{r}+\frac{1}{0} \times w \bar{s} r-0 \times \bar{w} s r \\
& +\frac{0}{0} \times \bar{w} \bar{s} r+\frac{0}{0} \times \bar{w} s \bar{r}+\frac{1}{0} \times w \bar{s} \bar{r}+\frac{0}{0} \bar{w} \bar{s} \bar{r} ; \\
p= & \frac{0}{0} \times w s r+1 \times w s \bar{r}+\frac{1}{0} \times w \bar{s} r-\frac{0}{0} \times \bar{w} s r \\
& +\frac{0}{0} \times \bar{w} \bar{s} r+\frac{0}{0} \times \bar{w} s \bar{r}+\frac{1}{0} \times w \bar{s} \bar{r}+\frac{0}{0} \bar{w} \bar{s} \bar{r} .
\end{aligned}
$$

The terms marked by $\frac{1}{0}$ are equal to zero and can therefore be eliminated, as follows:

$$
\begin{aligned}
t= & 1 \times w s r+1 \times w s \bar{r}-0 \times \bar{w} s r \\
& +\frac{0}{0} \times \bar{w} \bar{s} r+\frac{0}{0} \times \bar{w} s \bar{r}+\frac{0}{0} \bar{w} \bar{s} \bar{r} ; \\
p= & \frac{0}{0} \times w s r+1 \times w s \bar{r}+\frac{0}{0} \times \bar{w} s r \\
& +\frac{0}{0} \times \bar{w} \bar{s} r+\frac{0}{0} \times \bar{w} s \bar{r}+\frac{0}{0} \bar{w} \bar{s} \bar{r} .
\end{aligned}
$$

It can now be postulated that $t \times p$ can be obtained by multiplying the right-hand sides both Equations (79) and (80).

This means that each of the six members of one of the two sides needs to be multiplied with each of the six members of the other of the two sides. The result is 36 products (that is, $6 \times 6$ ).

However, all but six of the 36 terms can be readily eliminated. Only those in which a term in one Equation is multiplied with the same term in the other Equation will survive.

For example, $w s r+w s \bar{r}=0$. Nothing can be $r$ and $\bar{r}$ at the same time. The same Equation with zero applies to 23 more products.

The result of the multiplication of (79) and (80) is therefore as follows:

$$
\begin{aligned}
t \times p= & 1 \times \frac{0}{0} \times w s r+1 \times 1 \times w s \bar{r}-0 \times \frac{0}{0} \times \bar{w} s r \\
& +\frac{0}{0} \times \frac{0}{0} \times \bar{w} \bar{s} r+\frac{0}{0} \times \frac{0}{0} \times \bar{w} s \bar{r}+\frac{0}{0} \times \frac{0}{0} \bar{w} \bar{s} \bar{r} .
\end{aligned}
$$

It seems obvious that the following possible products of coefficients can be resolved as follows:

$$
\begin{gathered}
0 \times 0=0 ; \quad 0 \times \frac{0}{0}=\frac{0}{0} ; 0 \times 1=0 ; \\
\frac{0}{0} \times 1=\frac{0}{0} ; \text { and } 1 \times 1=1 .
\end{gathered}
$$

In other words, multiplying 0 by 0 results in 0 and multiplying by 1 retains the other member of the product. Both these principles apply simultaneously in the product $0 \times 1=0$.

The purport of these results is readily understood. One example. If $t$ includes everything (1) of wsr and $p$ does too, then anything that is both $t$ and $p$ will too.

Accordingly, Equation (81) can be abbreviated as follows:

$$
\begin{aligned}
t \times p= & \frac{0}{0} w s r+w s \bar{r}+\frac{0}{0} \times \frac{0}{0} \times \bar{w} \bar{s} r \\
& +\frac{0}{0} \times \frac{0}{0} \times \bar{w} s \bar{r}+\frac{0}{0} \times \frac{0}{0} \bar{w} \bar{s} \bar{r} .
\end{aligned}
$$


Five terms have survived. Three of them remain to be interpreted. They are the three terms signified by $\frac{0}{0} \times \frac{0}{0}$. How can $\frac{0}{0} \times \frac{0}{0}$ be resolved into a single coefficient? Is the single coefficient 0,1 , or $\frac{0}{0}$ ? It would seem tempting to declare something like

to be the case.

$$
\frac{0}{0} \times \frac{0}{0}=\frac{0}{0}
$$

However, the interpretation of $\frac{0}{0} \times \frac{0}{0}$ appears to require a more sophisticated approach. It will not be possible to elucidate all the ramifications of this more sophisticated approach. Still, some conclusions are possible and I believe them to be mathematically precise.

All depends on the relation between the domains of $\bar{w} \bar{s} r, \bar{w} s \bar{r}$, and $\bar{w} \bar{s} \bar{r}$ occupied by $t$ and those occupied by $p$. They are different possibilities.

Two principal possibilities are as follows. Either the two domains overlap or they do not overlap. If the domains do not overlap then the following Equation applies:

$$
\frac{0}{0} \times \frac{0}{0}=\frac{0}{0}
$$

In other words, if $p$ occupies an indefinite amount $\left(\frac{0}{0}\right)$ of a class and $t$ occupies an indefinite amount $\left(\frac{0}{0}\right)$ of the same class and the two domains do not overlap, then nothing (0) of that class is both $t$ and $p$.

As regards the remaining instances of $\frac{0}{0} \times \frac{0}{0}$, it needs to be established that $t$ and $p$ do not both overlap with all of a certain class. In such a case, the following Equation would apply:

$$
\frac{0}{0} \times \frac{0}{0}=1
$$

The task at hand is therefore to evaluate the three classes $\bar{w} \overline{s r}, \bar{w} s \bar{r}$, and $\bar{w} \bar{s} \bar{r}$ in terms of $t$ and $p$. It is obvious that each of these three classes consists of four combination classes, for a total of 12 . They are the following:

$$
\begin{aligned}
& \bar{w} \bar{s} r t p, \bar{w} \bar{s} r \bar{t} p, \bar{w} \bar{s} r t \bar{p}, \text { and } \bar{w} \bar{s} r \bar{t} \bar{p} \\
& \bar{w} s \bar{r} t p, \bar{w} s \bar{r} \overline{t p}, \bar{w} s \bar{r} t \bar{p} \text {, and } \bar{w} s \bar{r} \bar{t} \bar{p} ; \text { and } \\
& \bar{w} \bar{s} \bar{r} t p, \bar{w} \bar{s} \bar{r} \overline{t p}, \bar{w} \bar{s} \bar{r} t \bar{p}, \text { and } \bar{w} \bar{s} \bar{r} \bar{t} \bar{p} .
\end{aligned}
$$

It is evident that all the combination classes that are neither $t$ nor $p$, namely $\bar{w} \bar{s} r t \bar{p}, \bar{w} s \bar{r} \bar{t} \bar{p}$, and $\bar{w} \bar{s} \bar{r} \bar{t} \bar{p}$ need to be eliminated. Only combination classes that are either $t$ or $p$ can be part of the make-up of a larger class that is itself a component of what is both $t$ and $p$.

At the same time, $\bar{w} \bar{s} r \bar{t} \bar{p}, \bar{w} s \bar{r} \bar{t} \bar{p}$, and $\bar{w} \bar{s} \bar{r} \bar{t} \bar{p}$ are not 0 according to (76). It follows that $t p$ cannot include "all" $(=1)$ of $\bar{w} \bar{s} r, \bar{w} s \bar{r}$, and $\bar{w} \bar{s} \bar{r}$.

That leaves the following nine components to be evaluated:

$$
\begin{aligned}
& \bar{w} \bar{s} r t p, \bar{w} \bar{s} r \overline{t p} \text {, and } \bar{w} \bar{s} r t \bar{p} ; \\
& \bar{w} s \bar{r} t p, \bar{w} s \bar{r} \overline{t p} \text {, and } \bar{w} s \bar{r} t \bar{p} ; \text { and } \\
& \bar{w} \bar{s} \bar{r} t p, \bar{w} \bar{s} \bar{r} \overline{t p} \text {, and } \bar{w} \bar{s} \bar{r} t \bar{p} .
\end{aligned}
$$

The present focus is on establishing whether $t$ and $p$ overlap in $\bar{w} \bar{s} r, \bar{w} s \bar{r}$, and $\bar{w} \bar{s} \bar{r}$.

This comes down to asking whether parts of $\bar{w} \bar{s} r$, $\bar{w} s \bar{r}$, and $\bar{w} \bar{s} \bar{r}$ are both $t$ and $p$, which is the same as asking whether $\bar{w} \bar{s} r t p, \bar{w} s \bar{r} t p$, and $\bar{w} \bar{s} \bar{r} t p$ are not 0 according to (76).

It appears that $\bar{w} \bar{s} r t p$ and $\bar{w} s \bar{r} t p$ are not 0 , whereas $\bar{w} \bar{s} \bar{r} t p$ is.

According to Equation (76), all that is at the same time $s, t$, and $p$ must also be $w$. The combination class $\bar{w} s \bar{r} t p$ is not $w$ and therefore equals 0 according to (76).

Since $\bar{w} \bar{s} r t p$ and $\bar{w} s \bar{r} t p$ are not 0 according to (76), it does not matter much whether $\bar{w} \bar{s} r \overline{t p}, \bar{w} \bar{s} r t \bar{p}$, $\bar{w} \bar{s} \bar{r} \overline{t p}$, and $\bar{w} \bar{s} \bar{r} \bar{t} \bar{p}$ are or are not 0 . But it so happens that they are not 0 according to (76).

Equation (82) can now be finalized as follows:

$$
t p=w s \bar{r}+\frac{0}{0} w s r+\frac{0}{0} \bar{w} \bar{s} r+\frac{0}{0} \bar{w} \bar{s} \bar{r} .
$$

This is the same result as the one obtained by G. Boole by other means [79].

It should be noted that, in G. Boole's version of the Equation, the coefficient of both $(1-w) s r$ and $(1-w) s(1-r)$ ought to be -0 (minus zero) rather than G. Boole's “+ 0 ".

The significance of the minus sign is not fully transparent to me. I too obtain it for classes that are signified by the coefficient 0 . It somehow seems to indicate that the class in question needs to be subtracted from all that is being considered.

\section{The Theory of Rational Human Intelligence as a Black Box Theory}

\subsection{On the Feasibility of a Complete and Final Mathematical Theory of Rational Human Intelligence}

A fatal obstacle to the construction of a theory of rational intelligence might appear to be that the activity of the brain pertaining to rational human intelligence and taking place on a microscopic scale is for the most part opaque. 
No one has any idea at this time how exactly the brain produces rational thought and language.

Incidentally, I consider rational thought and language to be a single phenomenon that might also be styled as rational-thought-and-language.

Then again, the microscopic activity inside the brain pertaining to rational human intelligence is exteriorized in empirical and observable ways.

Rational human intelligence is exteriorized by sound waves issuing from the human speech organs or by symbols written in ink on a page. These sounds and written symbols by themselves do not carry any meaning or thought. They only produce meaning or thought when they are picked up by a hearing or seeing brain that knows to which meanings or thoughts the auditory or visual imprints inside the brain corresponding to those sounds or symbols outside the brain are linked.

The brain in question must be one that knows the specific language of the sounds and symbols.

Chinese sounds or writing mean nothing to a brain that does not know spoken or written Chinese. Sounds produce auditory imprints inside the brain. Those auditory imprints must be linked somehow to a meaning or thought. If they are not, then that means that the brain in question does not know the language.

It is abundantly clear, then, that rational human intelligence is exteriorized. It is therefore in a way accessible to independent observers that know the language in which it is expressed.

It is clear that the biochemical platform in which rational human intelligence is stored inside the brain must look very different from the sound and written platforms in which rational human intelligence is exteriorized in sounds.

Still, there is every reason to believe that both exhibit the same structure. And it is proposed here that this structure is entirely digital.

What leads one to believe that the biochemical platform and the exterior platform must be the same from the point of view of structure? There are many indications.

One indication is as follows. Speakers of the same language generally understand one another when they speak. A structure originating in one brain is exteriorized in sound waves, travels through the air, and is picked up by another brain. Little or nothing is lost in the process.

Also, from listening to their own words and seeing their own writing, speakers of a language can verify at any time that what they say or write reflects what they think inside their head. It is difficult to imagine that one would at all times be in danger of saying something that is different from what one thinks that one is saying without even knowing it. Even someone who is lying knows what the meaning is of what he is saying.

In reconstructing a theory of rational human intelli- gence, there is a distinction between the general mathematical principles at work and the specific biochemical model. As was noted above, I believe that the general mathematical principles can be formulated in their entirety. No such completeness is necessary about the precise biochemical mechanism.

It might perhaps come as a surprise that anything at all can be said about the mechanism. It is like describing an electric motor as a mechanism judging only from its outside effect, namely to produce motion.

Then again, the outside effect of rational human intelligence is much more variegated than the effect of an electric motor. In fact, the electric motor has in the end the exact same effect as the steam engine. That is an indication that one would be able to say almost nothing of the constitution of the two mechanisms judging from what they do.

\subsection{A Comparison with J. C. Maxwell's Theory of Electrodynamics}

It may defy belief that a complete and final mathematical theory of all of rational human intelligence is at all possible at this time (as I am convinced that it is) without anyone having any idea how the physical brain functions when it engages in rational human intelligence. It may therefore be encouraging to learn that something quite like this has already been done.

For example, there has been for some time a generally accepted mathematical theory of gravity. And yet, no one knows what gravity is.

In any event, the theory of gravity is not complete. A closer parallel to the theory that is proposed in follow-up papers is J. C. Maxwell's theory of electrodynamics.

The electromagnetic force is the only one of the four forces known to physics that is completely understood. And this complete understanding is owed to James Clerk Maxwell (1831-1879), who formulated the theory describing the force more than 140 years ago in the canonical form that it still has today [80].

The four forces, arranged in order of size of strength from high to low, are as follows: 1) strong; 2) electromagnetic; 3) weak; and 4) gravitational. The fundamental laws of electrodynamics are summed up in a handful of Equations produced by J. C. Maxwell.

A comparison of the theory proposed below with J. C. Maxwell's theory may serve to clarify the nature and character of the former. There are four characteristics that the theory that will be proposed for rational human intelligence shares with J. C. Maxwell's theory and there are three characteristics by which it differs.

But before describing these seven characteristics in detail, one similarity between the two theories needs to be dismissed as irrelevant. 
J. C. Maxwell's theory deals with electromagnetism. And rational human intelligence has in the end also to do with electromagnetism. It has been said that "it is scarcely an exaggeration to say that we live in an electromagnetic world - for virtually every force we experience in everyday life, with the exception of gravity, is electromagnetic in origin" [81].

The fact that rational human intelligence is apparently in the end electromagnetic in nature does not necessarily mean anything in relation to J. C. Maxwell's theory because most everything interesting in the human experience is electromagnetic, as it were. In fact, J. C. Maxwell's theory is all about measuring electromagnetism directly in its effects. No such measurements are part of the theory of rational human intelligence presented below.

Such measurements would be desirable if the aim were to determine the power expended in watts of a certain amount of effort of rational thinking. There is no doubt that energy is expended in thinking.

For comparison, a laborer working an eight-hour day might perhaps expend 75 watts or so. It would therefore be of interest to be able to determine exactly the amount of power expended in a certain event of rational human thinking.

In fact, to some extent, the energy that the brain consumes can already be measured somewhat precisely. But measuring the tiny amount that it takes to produce, say, a single well-constructed sentence is another matter. Presumably, some day, it will be possible to measure everything that happens in the brain precisely.

In any event, any such measurements are not relevant to the present paper's main argument. The amount of rational human thought is a different concern than the structure of rational human thought. The concern of the present paper is entirely with the latter.

\subsection{J. C. Maxwell's Theory and the Theory of Rational Human Intelligence: Four Shared General Characteristics}

The design of the four shared general characteristics listed in the present section and the differentiating characteristics listed in the next section is to classify the theory of rational human intelligence in a general way.

First shared characteristic-Quite in general, both J. C. Maxwell's theory and the theory of rational human intelligence are theories of physics. They explain a facet of physical reality in mathematical terms. The two facets in question are the propagation of rational human intelligence in the brain and the propagation of electromagnetic forces.

Second shared characteristic - Both facets of physical reality, electromagnetism and rational human intelligence, can be fully explained without any reference to what the facets actually are in the flesh, as it were.

It is like describing something that sits in a black box without being able to look inside the box. Naturally, since J. C. Maxwell, electromagnetism as a physical reality has come to be much better understood. But this understanding has not in the least changed his theory. Likewise, I believe that the theory proposed below will essentially not change, even when a better understanding is obtained as to what rational human intelligence in fact is in the flesh inside the brain.

It is in the end as unnecessary to J. C. Maxwell's theory to know what electromagnetism physically is as it is unnecessary to the theory proposed below to know what rational human intelligence physically is.

J. C. Maxwell's theory was formulated in its definitive form even though its author or anyone coming before him had no knowledge whatsoever of the existence of the electron or of the structure of the atom in general.

Electrical current is now typically presented in textbooks of all levels as a flow of electrons that have been detached from their atoms.

J. C. Maxwell had no notion of electrons. He could only observe electricity and magnetism in all their exterior effects without knowing which events produced the effects in question. When it comes to the observation of the effects of electricity and magnetism, he acknowledges a profound debt to the vast opus of Michael Faraday (1791-1867).

M. Faraday's work only confirms that there are definitely cases in which it is possible to learn an enormous amount about the nature of a phenomenon by observing it only in its external effects.

Not only did M. Faraday have no notion of what electricity or magnetism really is (even if he established for the first time much of what it does), his knowledge of mathematics apparently also did not extend much beyond trigonometry. It was J. C. Maxwell who cast these observations in a definitive mathematical form.

In regard to the theory of rational human intelligence, the brain is a little bit like a black box. One knows the inputs and the outputs of the box, that is, what goes into the box and what comes out of the box. But one does not really know the internal mechanism of the box.

Still, certain well-defined proposals about the internal biochemical mechanism of the box will be made in what follows. Furthermore, there should be a certain type of flawless correlation between what comes out of the box and what is inside it. After all, as was noted above, when speaking, one has a definite sense that one's words match what one is thinking.

In the same way, the system of electricity and magnetism was like a black box to J. C. Maxwell when he formulated his complete theory of the electromagnetic 
force.

In a recent biography of J. C. Maxwell by B. Mahon, it is called exactly that, a "black box-if you knew the inputs and could calculate the outputs without knowledge of the internal mechanism", and the author of the biography quotes J. C. Maxwell's own "more picturesque" description of the black box, which may be repeated here [82]:

In an ordinary belfry, each bell has one rope which comes down through a hole in the floor to the bellringer's room. But suppose that each rope, instead of acting on one bell, contributes to the motion of many pieces of machinery, and that the motion of each piece is determined not by the motion of one rope alone, but by that of several, and suppose, further, that all this machinery is silent and utterly unknown to the men at the ropes, who can only see as far as the holes above them.

The author of the biography further comments as follows:

[T] his was exactly what [J. C. Maxwell] needed. Nature's detailed mechanism could remain secret, like the machinery in the belfry.

This property of J. C. Maxwell's theory is adduced here in support of the notion that access to an internal mechanism is not a necessary obstacle to construct a complete and final theory of that mechanism.

A few additional quotes from the same biography further describe the black box nature of the theory.

(J. C. Maxwell) therefore chose the second route, which was to go beyond geometrical analogy and make an imaginary mechanical model of the combined electromagnetic field-a mechanism that would behave like the real field. If he could devise a suitable model, the equations governing its operation would also apply to the real field [83].

(He) was at pains to point out that this bizarre arrangement... was merely a model [84].

(His theory was) a completely new type of theory, one which admits that we may never understand the detailed workings of nature [85].

Even the most enlightened of his contemporaries thought that the next step should be to refine the model, to try to find the true mechanism. But perhaps he was already sensing that the ultimate mechanisms of nature may be beyond our powers of comprehension [86].

Third shared characteristic-Like J. C. Maxwell's theory, the theory of rational human intelligence is completely mathematical. As mathematical theories, both account for all possible cases.

Fourth shared characteristic-Like J. C. Maxwell's theory, the theory proposed of rational human intelligence is believed to be more or less complete. J. C. Maxwell's theory is more than 140 years old. In that regard, it is noteworthy that it was already complete when it was first formulated.

As was noted above, the electromagnetic force is the only one that is completely understood. As such, the theory of electromagnetism has served ever since as a model for the construction of other theories of physics.

In the introduction to one college handbook of electrodynamics, one reads the following [87]:

The laws of classical electrodynamics were discovered in bits and pieces by Franklin, Coulomb, Ampère, Faraday, and others, but the person who completed the job, and packaged it all in the compact form it has today, was James Clerk Maxwell.

A little over halfway through the same book, one reads in an "Intermission" [88]:

All of our cards are now on the table, and in a sense my job is done. In the first seven chapters, we assembled electrodynamics piece by piece, and now, with Maxwell's equations in their final form, the theory is complete. There are no more laws to be learned, no further generalizations to be considered.

\subsection{Three Differentiating General Properties}

First differentiating property-J. C. Maxwell's theory is entirely about quantity. By contrast, quantity plays no role in the theory of rational human intelligence.

The principal tool of measuring quantity in J. C. Maxwell's theory is the partial differential Equation. The introduction of partial differential Equations into the description of physical reality constitutes something of a turning-point in physics.

A. Einstein has describe the role of the partial differential Equation as follows [89]:

Before Maxwell people thought of physical reality - in so far as it represented events in nature-as material points, whose changes consist only in motions which are subject to total differential equations. After Maxwell they thought of physical reality as represented by continuous fields, not mechanically explicable, which are subject to partial differential equations. This change in the conception of reality is the most profound and the most fruitful that physics has experienced since Newton.

Even though the propagation of rational human intelligence is electrochemical, partial differential Equations or quantity in general play no role in the mathematical theory of rational human intelligence itself.

Second differentiating property-J. C. Maxwell's writings contain only few reflections about what electricity and magnetism physically are. As was noted before, J. C. Maxwell did not even know what an electron is.

The theory proposed below can likewise exist with only little reference to what rational human intelligence physically is. Still, rational human intelligence must have a physical structure. This physical structure will be elu- 
cidated as the biological structure of the brain comes to be better understood. In that regard, some proposals will be made below as to what that physical structure actually might be.

Third differentiating property-As distinct from J. C. Maxwell's theory and all other theories of physics, the theory of rational human intelligence as a theory of physics exhibits a unique property. It is reflexive. This reflexivity has already been discussed above. It takes acts of rational human intelligence to explain what rational human intelligence is. In that regard, rational human intelligence needs to explain itself, a bit like a snake biting its own tail.

\subsection{G. Boole and J. C. Maxwell}

J. C. Maxwell's theory has been adduced above for the purpose of providing a better characterization of the theory of rational human intelligence, which owes so much to G. Boole.

It appears that G. Boole (1815-1864) and J. C. Maxwell (1831-1879) exhibit a certain striking propinquity in relation to the three main coordinates of the human condition: person, space, and time.

As regards person, both were scientists endowed with exceptional skills in mathematics. And mathematics played a dominant role in their lives. Among many shared attributes pertaining to their scientific personas, both were members of the Royal Society in London. One differentiating feature is that G. Boole was entirely autodidact whereas J. C. Maxwell was not.

As regards time, both flourished around and about the middle part of the nineteenth century.

As regards space, G. Boole grew up in Lincolnshire in the east of Middle England and J. C. Maxwell somewhat north of that in the Galloway region of southwest Scotland.

It is otherwise a matter of coincidence that both died at about the same age, just short of a half-century lifespan.

The present focus is on two revolutionary theories, J. C. Maxwell's theory of electromagnetism and waves and G. Boole's theory of digital mathematics. As the result of a major revolutionary process in civilization of the last hundred years or so, all sorts of devices including satellites now emit and receive all kinds of information in digital format through the medium of radio waves.

These and many related events constitute in large part the combined application of two theories, J. C. Maxwell's theory of electromagnetism and G. Boole's theory of digital mathematics.

It is intriguing that the two theories came about not all that far from one another in place and time, more precisely in England, Ireland, and Scotland in the two decade period lasting from about 1845 to about 1865 .
It is therefore difficult to resist the temptation to search for any possible connections between the two theories and in a more general sense between the originators of the theories.

First the theories. It may be useful to preface the examination of possible relations between J. C. Maxwell's theory and G. Boole's theory by singling out a characteristic that they share.

This characteristic has in the end nothing to do with the contents of the theories. But it has everything to do with how the theories have been perceived by many.

The characteristic in question is that both theories were considered as being not quite scientific, that is, as mysterious or mystical or the like, by well-established scientists. May it suffice to adduce one example for each.

In his Preface to the re-edition of J. C. Maxwell's "A Dynamic Theory of the Electromagnetic Field", Th. T. Torrance cites a letter kept at the University of Glasgow in which the eminent physicist W. Thomson (1824-1907), Lord Kelvin, opined that J. C. Maxwell had lapsed into "mysticism" with his theory of electromagnetism [90].

J. Venn, G. Boole's principal disciple, notes that W. S. Jevons (1835-1882), the eminent logician, who was intimately acquainted with G. Boole's theory, saw need to excise from the theory all that is "obscure", "anomalous", "mysterious", or "dark" [91].

G. Boole's case was not helped by the fact that his wife, Mary Boole, who outlived him by 52 years, dying in 1916, by all accounts a most remarkable woman well ahead of her time who had raised five remarkable daughters, was a prolific author whose work often exhibited mystical tendencies and incorporated her husband's ideas in unorthodox ways.

Her Collected Works of 1931 are described in D. MacHale's biography of G. Boole as fifteen hundred pages containing an extraordinary mixture of insight, common-sense, perception, educational innovation, longwinded banality, incoherent confusion between philosophy and mathematics and, it must be stated, what at times appears to be complete nonsense [92].

I am otherwise not sure what D. MacHale exactly means when he calls G. Boole "a mystic genius" on the dust jacket of his biography of G. Boole. I assume that the term "mystic" here evokes the perplexity involved in trying to comprehend the origins of G. Boole's genius rather than any property of G. Boole's writings.

There is no doubt that both J. C. Maxwell's and G. Boole's theories were far off the beaten track. But the theories over time shed all appearance of the mystical.

In the case of J. C. Maxwell, recognition of the soundness of his theory came more than a century ago in the form of such developments as the positive verification of electromagnetic waves by $\mathrm{H}$. Hertz and the universally accepted notion that his Equations brought physics to the 
doorstep of the relativity and quantum theories.

In the case of G. Boole, the same kind of recognition came some decades later, namely in the 1930s with the advent of the Digital Age in electrical engineering and computing.

It would appear that it has taken a long time for J. C. Maxwell to emerge in modern physics as the pivotal figure between I. Newton, on the one hand, and A. Einstein and M. Planck, on the other hand. And it does not seem as if this notion has fully permeated into the common public consciousness.

The author of a recent biography, B. Mahon, finds this curious and the title of his autobiography, "The Man Who Changed Everything: The Life of James Clerk Maxwell", makes it obvious that he fully intended to do something about it, characterizing J. C. Maxwell as someone who "changed our perception of reality" [93].

In the case of G. Boole, there is a general awareness of his importance in computer science and electrical engineering. Still, the present paper and its continuation are based on the premise that there is every reason to consider G. Boole's theory as pivotal in its own domain as J. C. Maxwell's is in modern physics and that G. Boole was someone who "changed our perception of" human intelligence.

Now back to the relation between J. C. Maxwell's theory and G. Boole's theory.

I have not engaged in detailed historical investigations. However, it seems safe to conclude that G. Boole almost certainly never learned anything at all of J. C. Maxwell's theory of electromagnetism.

He would in fact hardly have had the opportunity. J. C. Maxwell's began his foundational study of electromagnetism around 1860. This study culminated in his "Dynamical Theory" of 1865 [94]. G. Boole died in 1864. Furthermore, G. Boole did not do original work in physics proper, and even J. C. Maxwell's colleagues were slightly bemused by his work in electromagnetism.

J. C. Maxwell was always engaged in all kinds of experiments. He was a laboratory man, in fact the founder of Cavendish Laboratory at the University of Cambridge. G. Boole never worked in a laboratory. He was a pure mathematician. By contrast, J. C. Maxwell only receives passing mention in histories of mathematics.

Then again, G. Boole was hardly disinterested in physics and there are even a few references to electricity in the index of his biography, partly relating to contacts with W. Thomson, Lord Kelvin [95].

As a teenager and a young man, G. Boole read and understood the writings on physics by I. Newton and P. S. Laplace and much else of what physics had to offer at the time. Like I. Newton, G. Boole was a native son of Lincoln in England. In fact, in 1835, as a young man living in Lincoln, G. Boole gave a highly technical and on some select points even critical public lecture on the "Genius and Discoveries of Sir Isaac Newton" [96].

It is otherwise tantalizing and even eerie how close G. Boole came to J. C. Maxwell's theory in space, time, and general subject matter.

G. Boole's final work on mathematics in the $1860 \mathrm{~s}$ concerned differential Equations [97], on which he wrote a textbook, including partial differential Equations, which play such a crucial role in J. C. Maxwell's theory.

In 1860-1865, J. C. Maxwell lived and worked in London. It was there that he conceived of his theory of electromagnetism [98]. As it happens, G. Boole was in London for some weeks from June 1864 onward and worked in the libraries of the Royal Society and the British Museum [99].

On October 27 of that year, J. C. Maxwell presented his essay on the "dynamical theory of the electromagnetic field" to the Royal Society [100]. The terms "dynamics" and "(partial) differential Equations" appear in the titles of articles by G. Boole dating to the early $1860 \mathrm{~s}$ [101].

But by October 1864, G. Boole was back in Cork, Ireland, where he was a professor of mathematics of what was then called the Queen's College. On November 24, he walked to a lecture in the rain and taught in wet cloths. As a result, he fell ill. On December 8, he died of his illness [102].

J. C. Maxwell's essay appeared in the next year, 1865.

When one considers that the marvel of satellites and cell phones beaming forth waves carrying digital information can be traced in great part to J. C. Maxwell and G. Boole, the fact that they perhaps did not bump into one another at the Royal Society or elsewhere in London in the summer of 1864 while both thoroughly immersed in (partial) differential Equations seems uncanny.

I do not know whether any sources exist that could document an encounter at that time or earlier.

This much for G. Boole and J. C. Maxwell's theory. Mere circumstances of time and space would in all probability have prevented G. Boole from being cognizant of J. C. Maxwell's theory even if he had shown interest.

But what about J. C. Maxwell and G. Boole's theory? No circumstances of time or space would have prevented J. C. Maxwell from knowing about G. Boole's theory.

G. Boole's two books on digital mathematics appeared in 1847 and 1854 when J. C. Maxwell (1831-1879) was a teenager and a young man. I have in fact been able to locate one statement in the works of A. Macfarlane- but nothing else so far anywhere else - to the effect that J. C. Maxwell, when a student at Edinburgh University in 1847-1850, became acquainted with G. Boole's Mathe- 
matical Analysis of Logic of 1847. But it will appear that there is an odd twist to the story.

A. Macfarlane (1851-1913) was a Scottish mathematician and physicist who emigrated to the United States and had an academic career at the University of Texas and then at Lehigh University.

A. Macfarlane is uniquely positioned because he was intimately acquainted with the works of both G. Boole and J. C. Maxwell, that is, with both mathematical logic and electricity.

In regard to G. Boole, he published an early work when he was about 28 about mathematical logic, already mentioned above, in which G. Boole's ideas are dominant [103]. In fact, it is obvious from this work that few have ever been as well acquainted with G. Boole's work on mathematical logic and probability theory as A. Macfarlane.

However, A. Macfarlane's relation to J. C. Maxwell is much closer. In fact, the relation was personal, whereas A. Macfarlane could not have known G. Boole in person. He wrote and published a dissertation on what exactly conditions the production of electric sparks. This work drew the attention of J. C. Maxwell, who successfully recommended A. Macfarlane for fellowship in the Royal Society of Edinburgh.

In 1901-1904, A. Macfarlane held lectures on British physicists and mathematicians at Lehigh University. These lectures were discovered in 1916. Ten lectures on physicists and ten lectures on mathematicians were deemed readily suitable for publication. G. Boole is included among the mathematicians and J. C. Maxwell is included among the physicists.

In the lecture on J. C. Maxwell, one finds the following one sentence statement about his student years at Edinburgh University [104]:

About this time George Boole published his Mathematical Analysis of Logic which found in Maxwell an appreciative reader.

We learn nothing more about J. C. Maxwell's interest in logic other than the intriguing statement that, as a student at Edinburgh, he "appears to have done most work for the class on logic".

The odd twist in the story anticipated above is as follows. In regard to his exposure to logic, J. C. Maxwell was a devoted student of the then famous logician W. Hamilton at the University of Edinburgh [105]. As is customary, I dutifully note that W. Hamilton needs to be distinguished from the contemporary Irish mathematician W. R. Hamilton, he of the quaternions, one of the most eminent mathematicians of his time.

The anticipated twist is that W. Hamilton's ideas on logic are very much diametrically opposed to G. Boole's. For example, W. Hamilton was very much opposed to the use of mathematics in logic and in fact to mathemat- ics in general as an educational discipline.

W. Hamilton had a well-known and rather intense exchange of ideas with A. De Morgan on the subject of logic.

G. Boole stayed neutral in public even if he clearly sided in private with A. De Morgan, who may well have been his closest correspondent [106].

In a letter to A. De Morgan of 1862, he writes the following about W. Hamilton [107]:

He and all his followers appear to me to have been trifling when writing about Logic. The notion that they have mapped out the whole kingdom of formed thought is a delusion that can only exist through ignorance-a kind of ignorance that prevails in no other subject.

To the extent that J. C. Maxwell ever retained any interest in logic after his student years, he may well have been deeply influenced by W. Hamilton, whose views on logic differ radically from G. Boole's alternative views involving digital mathematics.

Any acquaintance on the part of J. C. Maxwell with G. Boole's digital mathematics would therefore constitute an encounter with views that completely subverted what he may have retained from what W. Hamilton had taught him.

It seems difficult to imagine that J. C. Maxwell was professionally interested in logic. Such interest might have brought G. Boole's digital mathematics to his attention. I know of no evidence that he was even aware of it.

However, I have not engaged in any detailed historical investigations. Again, J. C. Maxwell was a man of the hands-on physical experiment. He was someone who, from a young age, wanted to know how physical contraptions worked. All his considerable mathematical skills were used in the service of this aim.

It may be concluded that G. Boole could hardly have known about J. C. Maxwell's theory but that J. C. Maxwell's expressed interested in G. Boole's. I otherwise know at this time of no evidence that the two men ever met. But the possibility can of course not be excluded.

It is significant to note that $\mathrm{G}$. Boole is not mentioned in the index of the recent biography of J. C. Maxwell [108] and J. C. Maxwell is not mentioned in the index of the recent biography of G. Boole [109].

There exists, however, at least one tenuous connection between J. C. Maxwell's physics and G. Boole's digital mathematics.

It is obvious that, in his digital mathematics, G. Boole uses the "+" symbol, the Boolean operator OR, differently from the way in which the sign is understood in quantitative mathematics.

J. Venn, G. Boole's principal disciple in matters of logic, argues in favor of using the symbol with similar but at the same time somewhat altered signification as a much preferable alternative to using an entirely new 
symbol.

The use of the same symbol acknowledges the existing similarity. In this regard, J. Venn points out that, even within mathematics, the symbol "+" can be used in a manner that is different from how it is understood in the simple expression " $1+1=2$ ", namely in the addition of vectors [110]. And he specifically refers to J. C. Maxwell's "Matter and Motion" as an example of such use [111].

It appears, then, that both G. Boole and J. C. Maxwell were comfortable with using the symbol "+" with a meaning that differs from what is by far the best known meaning that "+" has in arithmetic.

If J. C. Maxwell could use "+" to add vectors, then what is wrong with G. Boole using it for the function OR?

One wished, of course, that a certain symbol would always have exactly the same meaning.

Still, I am personally as convinced as J. Venn was that, in taking advantage of the similarity and avoiding an entirely new set of symbols, the benefits decisively outweigh the disadvantages.

One of the advantages is that the similarity conveys and evokes the perfect unity of all facets of rational human intelligence, including quantitative mathematics, digital mathematics, and the specific digital mathematics by which rational human intelligence operates.

I am otherwise also aware that a veritable Tower of Babel has been erected in relation to the notation of logical relations since G. Boole's time. It almost seems as if every prominent logician has his own notation.

And yet, I have otherwise never spotted any defects in G. Boole's algebra that would warrant its abandonment. It seems eminently felicitous, consistent, and easy to use. And using the same notation as other forms of mathematics and physics suggests the perfect unity of rational human intelligence and all its applications.

Since there are no direct connections, neither between the two men nor between their theories, it may be useful to look for indirect connections.

It may seem at first sight as if their theories deal with quite different subject matters and that it would seem in vain to seek any connections between them.

But there are certain indirect associations between the two theories. There are also indirect connections between the two men, in the form of third parties with which they were both in communication. Suffice it to adduce here what is perhaps the most striking example of both the former and the latter. The focus will be on the Turin native Joseph-Louis Lagrange (1736-1813) and the Scotsman William Thomson (1824-1907), Lord Kelvin.

It has been written about J.-L. Lagrange that he is "generally regarded as the keenest mathematician of the eighteenth century" [112].
The name J.-L. Lagrange is one that appears at critical junctures in the lives and works of both G. Boole and J. C. Maxwell. May it suffice to point here to the entry "Lagrange" in the index at the back of the biographies of the two men.

J. C. Maxwell's theory of electromagnetism and G. Boole's digital mathematics are remarkably similar in sharing two crucial characteristics.

First, they exhibit an absolute faith in the possibility of representing physical reality in purely mathematical form.

Second, they are both black box theories: J. C. Maxwell described electromagnetism without knowing what an electron is; G. Boole described human intelligence without knowing what a neuron or nerve cell is.

Much of J.-L. Lagrange's applications of differential Equations to physical reality exhibits the exact same characteristics. I believe that the two theories are in both regards deeply indebted to J.-L. Lagrange's thinking.

It is otherwise certain that both men were intimately acquainted with J.-L. Lagrange's work. For example, in the afore-mentioned summer of 1864, G. Boole traveled to London in part to study the original memoirs of J.-L. Lagrange, just at the time when J. C. Maxwell was in the same city for the first time applying J.-L. Lagrange's partial differential Equations to electromagnetism.

G. Boole wrote prolifically and with great originality on differential Equations. J. C. Maxwell applied them.

In sum, J.-L. Lagrange deeply influenced the writings of both J. C. Maxwell and G. Boole.

But when it comes specifically to J. C. Maxwell's theory of electromagnetism and G. Boole's digital mathematics, J.-L. Lagrange's influence is much more immediately apparent and direct in J. C. Maxwell's theory than it is in G. Boole's digital mathematics.

J.-L. Lagrange's Mécanique analytique is cited, and Equations are directly borrowed from it, at crucial junctures in J. C. Maxwell's writings on electromagnetism. What J. C. Maxwell did was to "extend Lagrange's method from mechanical to electromagnetic systems" and "the keynote of Lagrange's method was that it treated the system being analysed like a 'black box', if you knew the inputs and could specify the system's general characteristics you could calculate the outputs without knowledge of the internal mechanism" [113].

The following pattern emerges pertaining to the relation between J.-L. Lagrange, J. C. Maxwell, and G. Boole.

When it comes to representing physical and mechanical phenomena by means of pure mathematics, J.-L. Lagrange directly and deeply influenced J. C. Maxwell's theory of electromagnetism.

$\mathrm{He}$ also directly and deeply influenced G. Boole's work on quantitative mathematics, which was concerned 
in great part with differential Equations. But his influence on G. Boole's digital mathematics cannot have been direct. Differential Equations are a part of quantitative mathematics.

Then again, G. Boole's ideas on the nature of rational thought are a black box theory involving great trust in the ability of pure mathematics to describe physical and mechanical phenomena. And so is J.-L. Lagrange's work on differential Equations, G. Boole's area of expertise in the domain of quantitative mathematics.

It seems reasonable to assume that, in regard to his ideas on rational human intelligence, G. Boole was inspired by his expertise in quantitative mathematics to trust pure mathematics to describe a physical phenomenon such as human intelligence.

This much for indirect connections between the theories. But what about indirect connections between the two men themselves?

I single out the fact that both corresponded extensively with W. Thomson, Lord Kelvin.

As more of a mathematical physicist than a pure mathematician, W. Thomson was intellectually much closer to J. C. Maxwell than to G. Boole. W. Thomson became a kind of dean of British science and communicated with many scientists.

Still, he had lifelong special relationships with both G. Boole and J. C. Maxwell. He met J. C. Maxwell first as a boy in Glasgow. He may have met G. Boole first at a meeting of the British Association for the Advancement of Science in Cambridge in 1845, perhaps earlier.

May it suffice to illustrate the special relationships that W. Thomson had with both men by one quote each from the biographies of the latter two.

[G. Boole] and Thomson became close friends and lifelong correspondents on mathematics and other matters. There is no doubt that Thomson stimulated Boole to produce more papers... and, perhaps more importantly, increased considerably Boole's new-found confidence in himself [114].

[W. Thomson] could see at once that the boy had a rare gift and the two struck up a friendship which lasted throughout Maxwell's life. Thomson was a man from whom ideas flew like sparks. He and Faraday were the two people whose work most influenced Maxwell's own [115].

A preliminary superficial search has not revealed direct evidence that $\mathrm{W}$. Thomson spoke to G. Boole about J. C. Maxwell or to J. C. Maxwell about G. Boole.

The design of the preceding historical notes has been to bring J. C. Maxwell's and G. Boole's theories a little closer to one another and thereby add to the hope that it should be possible to describe the physical phenomenon of rational human intelligence entirely in purely mathematical terms.

\section{Acknowledgements}

I am grateful to an anonymous reviewer for helpful comments. The reviewer ventured to guess that the article results from a dissertation. But it is rather just the first installment of what is supposed to be several. It is not quite certain how additional installments will take shape. But the hope is that any such forthcoming will propose a more or less complete account of rational human intelligence.

While the preceding pages are concerned with intelligence, I believe-with Georges-Louis Leclerc, Comte de Buffon - that, in the end, style is everything and models are desirable. In that regard, Giovanni Battista Pergolesi has been inspiring. Traveler and author Charles Burney described his style as singularly clear, simple, and true, in addition to being sweet. Bach seemed like too many notes for the occasion.

The more I do mathematics, the more it seems to me as if the search for understanding is a pursuit of the adage "Know Thyself". It was my unexpected confrontation with J. C. Maxwell's work that encouraged me to at least try and tackle the topic of rational human intelligence comprehensively rather than piecemeal and I owe some valuable hints in relation to electrodynamics to Rei Ukita, an undergraduate student in engineering at Brown who took my ancient Egyptian literature class. This confrontation was a bit of a Proustian moment, less important for its contents than for its trigger effect, enhanced by a visit to Illiers-Combray in early June 2013, after the cathedrals at Amiens, Beauvais, and Chartres (dutifully listening to the sacred music of Pergolesi at each station) and before the Loire Valley castles at Chenonceaux, Cheverny, and Chambord and elsewhere. Let others judge what to make of this attempt to chercher la vérité en moi "seeking the truth in myself" when en face de quelque chose qui n'est pas encore "facing something that is not yet". If others too give it a try, we will at least be able to compare notes.

\section{REFERENCES}

[1] L. Depuydt, "Questions and Related Phenomena in Coptic and in General: Final Definitions Based on Boole's Laws of Thought," In: J. E. Goehring and J. E. Timbie, Eds., The World of Ancient Christianity, The Catholic University of America Press, Washington DC, 2007, pp. 72-94.

[2] L. Depuydt, "The Other Mathematics," Gorgias Press, Piscataway, 2008, pp. 307-321.

[3] L. Depuydt, "The Conjunctive in Egyptian and Coptic: Towards a Final Definition in Boolean Terms," In: C. G. Häberl, Ed., Proceedings of the 35th Annual Meeting of the North American Conference on Afroasiatic Linguistics (NACAL 35), Cambridge Scholars Publishing, New- 
castle Upon Tyne, 2009, pp. 13-30.

[4] L. Depuydt, "Towards the Full Digitalization of Grammar: The Case of the Egyptian and Coptic Nominal Sentence," Lingua Aegyptia, Vol. 17, 2010, pp. 27-50.

[5] L. Depuydt, "From 'My Body' to 'Myself' to 'Me Too': Philological and Digital Analysis of a Triple Shift in Egyptian," Journal of the American Research Center in Egypt, Vol. 45, 2009, pp. 247-290.

[6] L. Depuydt, "Zur unausweichlichen Digitalisierung der Sprachbetrachtung: 'allein', 'anderer', 'auch', 'einziger', '(seiner)seits', und 'selbst' als digitales Wortfeld im Ägyptisch-Koptischen und im Allgemeinen,” In: A. I. Blöbaum, K. Butt and I. Köhler, Eds., Lexical Fields, Semantics and Lexicography, Aegyptiaca Monasteriensia, Vol. 7, Shaker Verlag, Aachen, 2011, pp. 5-38.

[7] J. C. Maxwell, "Matter and Motion," Dover Publications Inc., New York, 1991 (first published in 1877).

[8] J. C. Maxwell, "A Treatise on Electricity and Magnetism," 2 Vols., 3rd Edition, Clarendon Press, Oxford, 1904 (1st Edition: 1873), vol. 1, p. xi.

[9] G. Boole, "An Investigation of the Laws of Thought, on Which Are Founded the Mathematical Theories of Logic and Probabilities," Walton and Maberly, London, 1854, pp. 24-27.

[10] J. Venn, "Symbolic Logic," 2nd Edition, Macmillan and Co., London and New York, 1894, pp. 453-476.

[11] J. Venn, "Symbolic Logic," 2nd Edition, Macmillan and Co., London and New York, 1894, pp. 499-500.

[12] G. W. Leibniz, "Opera Philosophica Quae Extant Latina Gallica Germanica Omnia," Scientia, Aalen, 1959 (facsimile of the original edition of 1840, supplemented with additional texts and a preface by R. Vollbrecht), p. 96.

[13] G. Boole, "An Investigation of the Laws of Thought, on Which Are Founded the Mathematical Theories of Logic and Probabilities," Walton and Maberly, London, 1854, p. 400.

[14] L. Carroll, "Symbolic Logic," edited, with annotations and an introduction, by W. W. Bartley, III, Clarkson N. Potter, Inc., New York, 1977 (original edition of Part I: 1896; of Part II: 1977), Part I, p. 150, No. 60 (Example), p. 180 , No. 60 (Solution).

[15] Ch. Petzold, "The Hidden Language of Computer Hardware and Software," Microsoft Press, Redmond, Washington, 2000, p. 87.

[16] L. Depuydt, "Synchrony and Diachrony: The Speaker/Observer Dichotomy," Göttinger Beiträge zur Sprachwissenschaft, Vol. 5, 2001, pp. 21-31.

[17] G. Boole, "An Investigation of the Laws of Thought, on Which Are Founded the Mathematical Theories of Logic and Probabilities," Walton and Maberly, London, 1854.

[18] Ch. Petzold, "The Hidden Language of Computer Hardware and Software," Microsoft Press, Redmond, Washington, 2000, p. 87

[19] Ch. Petzold, "The Hidden Language of Computer Hardware and Software," Microsoft Press, Redmond, Washington, 2000, p. 87.

[20] Ch. Petzold, "The Hidden Language of Computer Hard- ware and Software," Microsoft Press, Redmond, Washington, 2000, p. 87.

[21] G. Boole, "An Investigation of the Laws of Thought, on Which Are Founded the Mathematical Theories of Logic and Probabilities," Walton and Maberly, London, 1854, pp. 114-149.

[22] J. Venn, "Symbolic Logic," 2nd Edition, Macmillan and Co., London and New York, 1894, p. 396.

[23] L. Carroll, "Symbolic Logic," Clarkson N. Potter, Inc., New York, 1977, p. 30.

[24] G. Boole, "An Investigation of the Laws of Thought, on Which Are Founded the Mathematical Theories of Logic and Probabilities," Walton and Maberly, London, 1854.

[25] J. Venn, "Symbolic Logic," 2nd Edition, Macmillan and Co., London and New York, 1894.

[26] J. Venn, "Symbolic Logic," 2nd Edition, Macmillan and Co., London and New York, 1894, p. 177.

[27] J. Venn, "Symbolic Logic," 2nd Edition, Macmillan and Co., London and New York, 1894, p. 176, Note 2.

[28] G. W. Leibniz, “Opera Philosophica Quae Extant Latina Gallica Germanica Omnia," Scientia, Aalen, 1959, p. 102.

[29] G. W. Leibniz, “Opera Philosophica Quae Extant Latina Gallica Germanica Omnia,” Scientia, Aalen, 1959, p. 102.

[30] G. W. Leibniz, "Opera Philosophica Quae Extant Latina Gallica Germanica Omnia,” Scientia, Aalen, 1959, p. 103.

[31] On J. H. Lambert's logical studies, see J. Venn, "Symbolic Logic," 2nd Edition, Macmillan and Co., London and New York, 1894, pp. xxxi-xxxvii.

[32] J. Venn, "Symbolic Logic," 2nd Edition, Macmillan and Co., London and New York, 1894, p. xxxi.

[33] G. Boole, "An Investigation of the Laws of Thought, on Which Are Founded the Mathematical Theories of Logic and Probabilities," Walton and Maberly, London, 1854, p. 120.

[34] G. Boole, "An Investigation of the Laws of Thought, on Which Are Founded the Mathematical Theories of Logic and Probabilities," Walton and Maberly, London, 1854, pp. $120-121$

[35] G. Boole, "An Investigation of the Laws of Thought, on Which Are Founded the Mathematical Theories of Logic and Probabilities," Walton and Maberly, London, 1854, pp. 99-113.

[36] L. Carroll, "Symbolic Logic," Clarkson N. Potter Inc., New York, 1977, p. 67.

[37] L. Carroll, "Symbolic Logic," Clarkson N. Potter Inc., New York, 1977, pp. 67-68.

[38] L. Carroll, "Symbolic Logic," Clarkson N. Potter Inc., New York, 1977, p. 68.

[39] G. Boole, “An Investigation of the Laws of Thought, on Which Are Founded the Mathematical Theories of Logic and Probabilities," Walton and Maberly, London, 1854, pp. $31,37$.

[40] T. Hailperin, "Boole's Logic and Probability," NorthHolland Publishing Company, Amsterdam, New York, Oxford, 1976, p. 85.

[41] P. E. B. Jourdain, "The Development of Theories of 
Mathematic Logic and the Principles of Mathematics," The Quarterly Journal of Pure and Applied Mathematics, Vol. 44, 1913, pp. 113-128.

[42] Ph. E. B. Jourdain, "The Development of Theories of Mathematic Logic and the Principles of Mathematics," The Quarterly Journal of Pure and Applied Mathematics, Vol. 41, 1910, pp. 324-352 (about G. Boole and some on G. W. Leibniz).

[43] Ph. E. B. Jourdain, "The Development of Theories of Mathematic Logic and the Principles of Mathematics," The Quarterly Journal of Pure and Applied Mathematics, Vol. 43, 1912, pp. 219-314 (on H. McColl, G. Frege, and G. Peano).

[44] P. E. B. Jourdain, "The Development of Theories of Mathematic Logic and the Principles of Mathematics," The Quarterly Journal of Pure and Applied Mathematics, Vol. 44, 1913, pp. 113-128, at p. 117.

[45] See now also I. Grattan-Guinness, "The Correspondence between George Boole and Stanley Jevons, 1863-1864," History and Philosophy of Logic, Vol. 12, 1991, pp. 15-35, at p. 30 .

[46] G. Boole, "An Investigation of the Laws of Thought, on Which Are Founded the Mathematical Theories of Logic and Probabilities," Walton and Maberly, London, 1854, pp. 130-131.

[47] G. Boole, "An Investigation of the Laws of Thought, on Which Are Founded the Mathematical Theories of Logic and Probabilities," Walton and Maberly, London, 1854, p. 139.

[48] D. MacHale, "George Boole: His Life and Work," Boole Press, Dublin, 1985, p. 71.

[49] J. Venn, "Symbolic Logic," 2nd Edition, Macmillan and Co., London and New York, 1894, pp. 175, 269.

[50] J. Venn, "Symbolic Logic," 2nd Edition, Macmillan and Co., London and New York, 1894, pp. xxxiii-xxxiv.

[51] E. Schröder, "Vorlesungen über die Algebra der Logik (Exakte Logik)," J. C. Hinrichs, Leipzig, Vol. 2, 1891/ 1905 , p. x. E. Schröder died in 1902. Part 2 of Vol. 2 was edited by E. Müller on behalf of the Deutsche Mathematiker-Vereinigung. All three volumes of E. Schröder's "Vorlesungen über die Algebra der Logik" have been reprinted in 2001 by Thoemmes Press, Bristol, England.

[52] E. Schröder, "Vorlesungen über die Algebra der Logik (Exakte Logik)," J. C. Hinrichs, Leipzig, Vol. 1, 1890, p. 460.

[53] E. Schröder, "Vorlesungen über die Algebra der Logik (Exakte Logik)," J. C. Hinrichs, Leipzig, Vol. 1, 1890, p. 481.

[54] E. Schröder, "Vorlesungen über die Algebra der Logik (Exakte Logik)," J. C. Hinrichs, Leipzig, Vol. 2, 1891/ 1905; E. Schröder, "Vorlesungen über die Algebra der Logik (Algebra und Logik der Relative)," J. C. Hinrichs, Leipzig, 1895, Vol. 3.

[55] E. Schröder, "Vorlesungen über die Algebra der Logik (Exakte Logik)," J. C. Hinrichs, Leipzig, Vol. 2, 1891/ 1905, p. 402.

[56] E. Schröder, "Vorlesungen über die Algebra der Logik (Algebra und Logik der Relative)," J. C. Hinrichs, Leip- zig, 1895, Vol. 3, p. 1.

[57] I. Grattan-Guinness, "The Search for Mathematical Roots, 1870-1940: Logics, Set Theories and the Foundations of Mathematics from Cantor through Russell to Gödel," Princeton University Press, Princeton and Oxford, 2000.

[58] I. Grattan-Guinness, "The Search for Mathematical Roots, 1870-1940: Logics, Set Theories and the Foundations of Mathematics from Cantor through Russell to Gödel," Princeton University Press, Princeton, 2000, p. 176.

[59] Review of G. Frege, "Begriffsschrift: Eine der arithmetischen nachgebildete Formelsprache des reinen Denkens," L. Nebert, Halle, 1879, in Zeitschrift für Mathematik und Physik, Vol. 25, 1880, pp. 81-94.

[60] G. Boole, "An Investigation of the Laws of Thought, on Which Are Founded the Mathematical Theories of Logic and Probabilities," Walton and Maberly, London, 1854, p. 72.

[61] G. Boole, "An Investigation of the Laws of Thought, on Which Are Founded the Mathematical Theories of Logic and Probabilities," Walton and Maberly, London, 1854, p. 123.

[62] G. Boole, “An Investigation of the Laws of Thought, on Which Are Founded the Mathematical Theories of Logic and Probabilities," Walton and Maberly, London, 1854, pp. $138-140$.

[63] L. Depuydt, "Higher Variations of the Monty Hall Problem $(3.0,4.0)$ and Empirical Definition of the Phenomenon of Mathematics, in Boole's Footsteps, as Something the Brain Does," Advances in Pure Mathematics, Vol. 2, No. 4, 2012, pp. 243-273, at pp. 268-272. www.scirp.org/journal/apm

[64] On the reception of G. Boole's ideas, see several papers in J. Gasser, Ed., "A Boole Anthology: Recent and Classical Studies in the Logic of George Boole," Kluwer Academic Publishers, Dordrecht, Boston, and London, 2000.

[65] G. Boole, "An Investigation of the Laws of Thought, on Which Are Founded the Mathematical Theories of Logic and Probabilities," Walton and Maberly, London, 1854, pp. $125-126$.

[66] J. Venn, "Symbolic Logic," 2nd Edition, Macmillan and Co., London, 1894, p. 301.

[67] J. Venn, "Symbolic Logic," 1st Edition, Macmillan and Co., London, 1881, pp. 222-239. Copies of the first edition are not easily to locate. An electronic copy is available at archive.org/details/symboliclogic00venniala (Accessed January 14, 2013); it is a scan of a copy kept at the University of California at San Diego.

[68] E. Schröder, "Vorlesungen über die Algebra der Logik (Exakte Logik),” J. C. Hinrichs, Leipzig, Vol. 1, 1890, p. 533.

[69] J. Venn, "Symbolic Logic," 2nd Edition, Macmillan and Co., London and New York, 1894, p. 301, Note 1.

[70] E. Schröder, "Vorlesungen über die Algebra der Logik (Exakte Logik),” J. C. Hinrichs, Leipzig, Vol. 1, 1890, p. 489.

[71] E. Schröder, "Vorlesungen über die Algebra der Logik (Exakte Logik)," J. C. Hinrichs, Leipzig, Vol. 1, 1890, p. 
481.

[72] E. Schröder, "Vorlesungen über die Algebra der Logik (Exakte Logik)," J. C. Hinrichs, Leipzig, Vol. 1, 1890, p. 489.

[73] E. Schröder, "Vorlesungen über die Algebra der Logik (Exakte Logik)," J. C. Hinrichs, Leipzig, Vol. 1, 1890, p. 480.

[74] A. Macfarlane, "Principles of the Algebra of Logic," David Douglas, Edinburgh, 1879, pp. 76-77.

[75] G. Boole, “An Investigation of the Laws of Thought, on Which Are Founded the Mathematical Theories of Logic and Probabilities," Walton and Maberly, London, 1854, pp. 90,92 .

[76] G. Boole, "An Investigation of the Laws of Thought, on Which Are Founded the Mathematical Theories of Logic and Probabilities," Walton and Maberly, London, 1854, p. 124.

[77] G. Boole, "The Mathematical Analysis of Logic, Being an Essay towards a Calculus of Deductive Reasoning," Macmillan, Barclay, \& Macmillan, Cambridge and George Bell, London, 1847. Reprinted with different pagination, though with references to the pagination of the original edition, in G. Boole, "Studies in Logic and Probability," Watts \& Co., London, 1952.

[78] J. Venn, "Symbolic Logic," 2nd Edition, Macmillan and Co., London and New York, 1894, p. 393, Note 1.

[79] G. Boole, "An Investigation of the Laws of Thought, on Which Are Founded the Mathematical Theories of Logic and Probabilities," Walton and Maberly, London, 1854, p. 139.

[80] First in J. C. Maxwell, "A Dynamical Theory of the Electromagnetic Field," edited and introduced by Th.F. Torrance, with an appreciation by A. Einstein, Scottish Academic Press, Edinburgh, 1982 (1st publication: 1865), described as "forever one of the finest of all man's scientific accomplishments" in B. Mahon, "The Man Who Changed Everything: The Life of James Clerk Maxwell," Wiley, Chichester, West Sussex, 2003, p. 119; later comprehensively in J. C. Maxwell, "A Treatise on Electricity and Magnetism," 2 Vols., 3rd Edition, Clarendon Press, Oxford, 1904 (1st Edition: 1873).

[81] D. J. Griffiths, "Introduction to Electrodynamics," Prentice Hall, Upper Saddle River, N.J., p. xii.

[82] B. Mahon, "The Man Who Changed Everything: The Life of James Clerk Maxwell," Wiley, Chichester, 2003, p. 121.

[83] B. Mahon, "The Man Who Changed Everything: The Life of James Clerk Maxwell," Wiley, Chichester, 2003, p. 96.

[84] B. Mahon, "The Man Who Changed Everything: The Life of James Clerk Maxwell," Wiley, Chichester, 2003, p. 104.

[85] B. Mahon, "The Man Who Changed Everything: The Life of James Clerk Maxwell," Wiley, Chichester, 2003, p. 110.

[86] B. Mahon, "The Man Who Changed Everything: The Life of James Clerk Maxwell," Wiley, Chichester, 2003, p. 120.

[87] D. J. Griffiths, "Introduction to Electrodynamics," Pren- tice Hall, Upper Saddle River, N.J., p. xii.

[88] D. J. Griffiths, "Introduction to Electrodynamics," 3rd Edition, Prentice Hall, Upper Saddle River, p. 343.

[89] J. C. Maxwell, "A Dynamical Theory of the Electromagnetic Field," Scottish Academic Press, Edinburgh, 1982, p. 31 .

[90] Th. F. Torrance, in J. C. Maxwell, “A Dynamical Theory of the Electromagnetic Field," Scottish Academic Press, Edinburgh, 1982, p. ix.

[91] J. Venn, "Symbolic Logic," 2nd Edition, Macmillan and Co., London and New York, 1894, p. xxviii.

[92] D. MacHale, "George Boole: His Life and Work," Boole Press, Dublin, 1985, p. 256.

[93] B. Mahon, "The Man Who Changed Everything: The Life of James Clerk Maxwell," Wiley, Chichester, West Sussex, 2003, back cover.

[94] J. C. Maxwell, "A Dynamical Theory of the Electromagnetic Field," Scottish Academic Press, Edinburgh, 1982.

[95] D. MacHale, "George Boole: His Life and Work," Boole Press, Dublin, 1985, p. 300.

[96] D. MacHale, "George Boole: His Life and Work," Boole Press, Dublin, 1985, pp. 34-38.

[97] For bibliography, see D. MacHale, "George Boole: His Life and Work," Boole Press, Dublin, pp. 281-282.

[98] B. Mahon, "The Man Who Changed Everything: The Life of James Clerk Maxwell," Wiley, Chichester, 2003, pp. 90-127.

[99] D. MacHale, "George Boole: His Life and Work," Boole Press, Dublin, 1985, p. 222.

[100] J. C. Maxwell, "A Dynamical Theory of the Electromagnetic Field," 1982, p. ix.

[101] D. MacHale, "George Boole: His Life and Work," Boole Press, Dublin, 1985, pp. 281-282.

[102] D. MacHale, "George Boole: His Life and Work," Boole Press, Dublin, 1985, pp. 240-243.

[103] A. Macfarlane, "Principles of the Algebra of Logic," David Douglas, Edinburgh, 1879.

[104] A. Macfarlane, "Lectures on Ten British Physicists of the Nineteenth Century," Mathematical Monographs, Vol. 20, John Wiley \& Sons, Inc., New York; Chapman \& Hall, Limited, London, 1919, p. 11.

[105] B. Mahon, "The Man Who Changed Everything: The Life of James Clerk Maxwell," Wiley, Chichester, 2003, p. 21.

[106] G. C. Smith, "The Boole-De Morgan Correspondence," Clarendon Press, Oxford, 1982.

[107] D. MacHale, "George Boole: His Life and Work," Boole Press, Dublin, 1985, p. 225.

[108] B. Mahon, "The Man Who Changed Everything: The Life of James Clerk Maxwell,” Wiley, Chichester, 2003.

[109] D. MacHale, "George Boole: His Life and Work," Boole Press, Dublin, 1985.

[110] J. Venn, "Symbolic Logic," 2nd Edition, Macmillan and Co., London and New York, 1894, p. xii.

[111] J. C. Maxwell, "Matter and Motion," Dover Publications, 
Inc., New York, 1991.

[112] C. B. Boyer, "A History of Mathematics," 2nd Edition, John Wiley \& Sons Inc., New York, 1989, p. 490.

[113] B. Mahon, "The Man Who Changed Everything: The Life of James Clerk Maxwell," Wiley, Chichester, 2003, p.
121.

[114] D. MacHale, "George Boole: His Life and Work," Boole Press, Dublin, 1985, p. 67.

[115] B. Mahon, "The Man Who Changed Everything: The Life of James Clerk Maxwell," Wiley, Chichester, 2003, p. 20. 